\title{
Abundance analysis of barium and mild barium stars ${ }^{\star} \star \star$
}

\author{
R. Smiljanic ${ }^{1, \star \star \star}$, G. F. Porto de Mello ${ }^{1}$, and L. da Silva ${ }^{2}$ \\ 1 Observatório do Valongo, Universidade Federal do Rio de Janeiro, Ladeira do Pedro Antônio 43, Saúde, \\ Rio de Janeiro-RJ 20080-090, Brazil \\ e-mail: rodolfo@astro.iag.usp.br; gustavo@ov.ufrj.br \\ 2 Observatório Nacional, Rua Gal. José Cristino 77, São Cristovão, Rio de Janeiro-RJ 20921-400, Brazil \\ e-mail: licio@on.br
}

Received 20 June 2006 / Accepted 13 March 2007

\begin{abstract}
Aims. We compare and discuss abundances and trends in normal giants, mild barium, and barium stars, searching for differences and similarities between barium and mild barium stars that could help shed some light on the origin of these similar objects. Also, we search for nucleosynthetic effects possibly related to the s-process that were observed in the literature for elements like $\mathrm{Cu}$ in other types of s-process enriched stars.

Methods. High signal to noise, high resolution spectra were obtained for a sample of normal, mild barium, and barium giants. Atmospheric parameters were determined from the Fe I and Fe II lines. Abundances for $\mathrm{Na}, \mathrm{Mg}, \mathrm{Al}, \mathrm{Si}, \mathrm{Ca}, \mathrm{Sc}, \mathrm{Ti}, \mathrm{V}, \mathrm{Cr}, \mathrm{Mn}, \mathrm{Fe}$, $\mathrm{Co}, \mathrm{Ni}, \mathrm{Cu}, \mathrm{Zn}, \mathrm{Sr}, \mathrm{Y}, \mathrm{Zr}, \mathrm{Ba}, \mathrm{La}, \mathrm{Ce}, \mathrm{Nd}, \mathrm{Sm}, \mathrm{Eu}$, and $\mathrm{Gd}$, were determined from equivalent widths and model atmospheres in a differential analysis, with the red giant $\epsilon$ Vir as the standard star.

Results. The different levels of s-process overabundances of barium and mild barium stars were earlier suggested to be related to the stellar metallicity. Contrary to this suggestion, we found in this work no evidence of barium and mild barium having a different range in metallicity. However, comparing the ratio of abundances of heavy to light s-process elements, we found some evidence that they do not share the same neutron exposure parameter. The exact mechanism controlling this difference is still not clear. As a by-product of this analysis we identify two normal red giants misclassified as mild barium stars. The relevance of this finding is discussed. Concerning the suggested nucleosynthetic effects possibly related to the s-process, for elements like $\mathrm{Cu}, \mathrm{Mn}, \mathrm{V}$ and $\mathrm{Sc}$, we found no evidence for an anomalous behavior in any of the s-process enriched stars analyzed here. However, further work is still needed since a clear $[\mathrm{Cu} / \mathrm{Fe}]$ vs. $[\mathrm{Ba} / \mathrm{Fe}]$ anticorrelation exists for other s-process enriched objects.
\end{abstract}

Key words. stars: abundances - stars: chemically peculiar - stars: late-type

\section{Introduction}

Barium stars are chemically peculiar $\mathrm{G}-\mathrm{K}$ giants first identified by Bidelman \& Keenan (1951). These stars were found to have the Ba II $4554 \AA$ resonance line, the $\mathrm{CH}$ G band and the Sr II $4077 \AA$ and $4215 \AA$ lines abnormally enhanced. Abundance analyses (Burbidge \& Burbidge 1957; Warner 1965) confirmed that such features were due to real atmospheric overabundances of carbon and of the heavy s-process elements.

S-process nucleosynthesis itself is only expected in thermally pulsing asymptotic giant branch (AGB) stars. Two reactions are the main providers of neutrons, ${ }^{22} \mathrm{Ne}(\alpha, \mathrm{n})^{25} \mathrm{Mg}$ and ${ }^{13} \mathrm{C}(\alpha, \mathrm{n}){ }^{16} \mathrm{O}$. Although the ${ }^{22} \mathrm{Ne}$ reaction was earlier thought to be dominant, it was shown (Tomkin \& Lambert 1979; Tomkin \& Lambert 1983; Malaney 1987a) that it produces results incompatible with the observations. Thus, the most important neutron source is now thought to be the ${ }^{13} \mathrm{C}$ reaction. The source of ${ }^{13} \mathrm{C}$, however, is not well established. Recent works on s-process enrichment usually parameterize the amount of ${ }^{13} \mathrm{C}$ burnt during

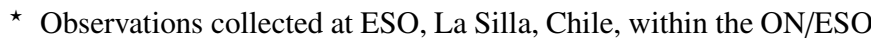
agreements.

$\star \star$ Tables $8-10$ are only available in electronic form at http://www. aanda.org

$\star \star \star$ Present address: Universidade de São Paulo, IAG, Dpt. de Astronomia, Rua do Matão 1226, São Paulo-SP 05508-900, Brazil.
}

the s-process operation (Busso et al. 1995, 2001; Gallino et al. 1998).

In AGBs, the deep dredge-up phenomena that follows the thermal pulses, the so-called third dredge-up, mixes some of the processed material to the atmosphere, where it becomes accessible to observations. Barium stars, however, are less massive and less luminous than the AGB stars. It is not expected for barium stars to synthetize s-process elements in their interiors or even to be able to dredge this material up to the surface, thus they cannot be self-enriched.

An important discovery that led to the solution of this problem was made in the early eighties. Through the radial velocity monitoring of a sample of barium and normal giants, it was discovered that all barium giants are likely members of binary systems (McClure et al. 1980; McClure 1983, 1984). White dwarf companions were also detected in the UV with the International Ultraviolet Explorer (IUE) (Böhm-Vitense 1980; Domini \& Lambert 1983; Böhm-Vitense \& Johnson 1985). More recent radial velocities monitoring (Udry et al. 1998a,b) and UV observations (Böhm-Vitense et al. 2000) with the Hubble Space Telescope (HST) have supported the idea of binarity.

The chemical peculiarities of the barium stars are then directly related to their binarity through a mass transfer scenario. The companion star seen today as a white dwarf was initially the more massive star of the system. As such it evolved faster and became a thermally pulsing AGB, while the current barium 
Table 1. Data of the sample stars. Visual magnitudes and spectral types are from the SIMBAD database. The effective temperatures $\left(T_{\text {eff }}\right), \log g$, metallicities and barium abundances are from Zacs (1994) for all stars with the exception of HR 1016 (Pilachowski 1977), HR 4932 (McWilliam 1990), and HR 5058 (Luck \& Bond 1991). The colors $(V-K)$ and $(R-I)$ are from Hoffleit \& Jaschek (1982) and Johnson (1966). The stars HR 440, HR 1326 and HR 4932 ( $\epsilon$ Vir) are the three normal giants included in the sample.

\begin{tabular}{cccccccccc}
\hline \hline HR & HD & $V$ & $(V-K)$ & $(R-I)$ & ST & $T_{\text {eff }}$ & $\log g$ & {$[\mathrm{Fe} / \mathrm{H}]$} & {$[\mathrm{Ba} / \mathrm{Fe}]$} \\
\hline 440 & 9362 & 4.0 & - & +0.51 & K0III-IV & - & - & - & - \\
649 & 13611 & 4.4 & +2.06 & +0.49 & G6II-III & 5050 & 2.3 & -0.3 & +0.44 \\
1016 & 20894 & 5.5 & - & +0.47 & G6.5IIb & 5100 & 3.6 & -0.2 & -0.20 \\
1326 & 26967 & 3.9 & +2.48 & +0.59 & K1III & - & - & - & - \\
2392 & 46407 & 6.2 & +2.21 & +0.48 & K0III & 5000 & 2.1 & +0.1 & +1.34 \\
4608 & 104979 & 4.1 & +2.22 & +0.49 & G8III & 5000 & 2.2 & -0.1 & +0.93 \\
4932 & 113226 & 2.8 & +2.04 & +0.45 & G8IIIab & 5060 & 2.97 & +0.15 & - \\
5058 & 116713 & 5.1 & - & - & K0.5III & 5000 & 3.0 & +0.2 & - \\
5802 & 139195 & 5.3 & - & +0.45 & K0III & 5140 & 2.7 & +0.3 & +0.52 \\
7321 & 181053 & 6.4 & - & - & K0IIIa & 4885 & 2.1 & -0.2 & +0.34 \\
8115 & 202109 & 3.2 & +2.11 & +0.48 & G8.5III & 5050 & 2.8 & +0.1 & +0.41 \\
8204 & 204075 & 3.7 & +1.88 & +0.43 & G4II & 5230 & 1.5 & +0.2 & +1.31 \\
- & 205011 & 6.4 & - & - & G9IIIa & 4950 & 2.4 & +0.1 & +0.88 \\
8878 & 220009 & 5.1 & - & - & K2III & 4575 & 2.6 & -0.1 & +0.42 \\
\hline
\end{tabular}

star was still in an earlier evolutionary stage. The star then enriched its He burning envelope with products of the s-process nucleosynthesis and through successive third dredge-ups mixed this material to the atmosphere. This enriched material was then transferred onto the current barium star through mass loss mechanisms (Jorissen \& Mayor 1992; Liang et al. 2000). Thus, the overabundances are not intrinsic to the barium star but are important observational tests of the theories of nucleosynthesis, convection and mass loss in cool stars and the study of the chemical evolution of the Galaxy.

Concerning the details of the s-process nucleosynthesis, there is some observational evidence pointing towards a possible preferential depletion of certain iron peak elements. Main sequence stars of the young Ursa Major moving group (UMaG hereafter), which are s-process enriched, show a $\mathrm{Cu}$ depletion with respect to $\mathrm{Fe}$ (Castro et al. 1999). Other barium enriched objects show the same behavior (Pereira \& Porto de Mello 1997; Pereira et al. 1998). Particularly, the abundance pattern of HR 6094 (Porto de Mello \& da Silva 1997), an UMaG member, suggests the depletion of $\mathrm{Mn}$ and $\mathrm{Cu}$ and that $\mathrm{V}$ and $\mathrm{Sc}$ could have been preserved with respect to Fe. Such results may represent important constraints to the neutron capture models in AGBs and deserve further investigation. This kind of data is still very scarce in the literature.

Detailed abundance analyses of barium (or mild barium) stars with modern high quality data are scarce in the literature (Boyarchuk et al. 2002; Liang et al. 2003; Antipova et al. 2004; Yushchenko et al. 2004; Allen \& Barbuy 2006). Most of the available analyses are based on data with lower $S / N$ ratio (Pilachowski 1977; Smith 1984; Kovacs 1985; Luck \& Bond 1991; Zacs 1994). Thus, abundance errors in these works could possibly be blurring the small scale of the suggested nucleosynthetic effects.

In this work we derived atmospheric parameters and the detailed chemical composition of a sample containing eleven stars classified in the literature as barium or mild barium stars and three normal giants for comparison purposes. Abundances were obtained for the light elements $\mathrm{Na}, \mathrm{Mg}, \mathrm{Al}, \mathrm{Si}, \mathrm{Ca}$; the iron peak elements $\mathrm{Sc}, \mathrm{Ti}, \mathrm{V}, \mathrm{Cr}, \mathrm{Mn}, \mathrm{Fe}, \mathrm{Co}, \mathrm{Ni}$; $\mathrm{Cu}, \mathrm{Zn}$ (considered as transition elements between the iron peak and s-process elements); the s-process elements $\mathrm{Sr}, \mathrm{Y}, \mathrm{Zr}, \mathrm{Ba}, \mathrm{La}, \mathrm{Ce}, \mathrm{Nd}$; and the r-process dominated elements $\mathrm{Sm}, \mathrm{Eu}, \mathrm{Gd}$.
The abundances of the barium and mild barium stars are compared and the possible nucleosynthetic effects discussed. In addition, we discuss the relevance of the identification in this work of two normal giants previously misclassified as mild barium stars. Such a result shows the necessity of high quality analysis for this class of peculiar stars. The observations are described in Sect. 2, the stellar parameters in Sect. 3 and the abundances in Sect. 4. In Sect. 5 we discuss the results and the nucleosynthetic effects while conclusions are drawn in Sect. 6.

\section{Observational data}

High resolution CCD spectra were obtained using the FEROS (Kaufer et al. 1999) spectrograph at the ESO $1.52 \mathrm{~m}$ telescope at La Silla, Chile. FEROS is a fiber-fed echelle spectrograph that provides a full wavelength coverage of $\lambda \lambda 3500-9200$ over 39 orders at a resolving power of $R=48000$. The detector used was an EEV CCD chip with $2048 \times 4096$ pixels and a pixel size of $15 \mu \mathrm{m}$. All spectra were reduced using the FEROS pipeline software. The spectra have a typical signal to noise ratio of $S / N \approx 500-600$.

The sample stars were selected based on previous literature analyses. It was a concern to include stars with different overabundances, ranging from mild barium stars to barium stars with overabundances larger than 1.0 dex. It is important to span such a variety of barium stars in order to search for possible correlations among the observed overabundances and the atmospheric and evolutionary parameters. Table 1 presents the literature data on the selected objects. All the stars, with the exception of HR 1016 (Pilachowski 1977) and HR 5058 (Luck \& Bond 1991), were analyzed by Zacs (1994) who conducted a detailed analysis of a larger number of barium stars but used data with much lower $S / N$ than ours.

We selected two normal giants, HR 440 and HR 1326, for comparison purposes, from the Bright Star Catalogue (Hoffleit \& Jaschek 1982). Judging by the color indices, their effective temperatures should be similar to those of the barium giants. The third normal star, HR 4932, is $\epsilon$ Vir, the same standard star used by Zacs (1994) and one of the most extensively studied giants in literature (Cayrel de Strobel et al. 2001). We also adopted $\epsilon$ Vir as the standard star for a differential analysis.

The analysis was conducted using equivalent widths ( $E W \mathrm{~s}$ hereafter). The $E W \mathrm{~s}$ were measured in previously normalized 


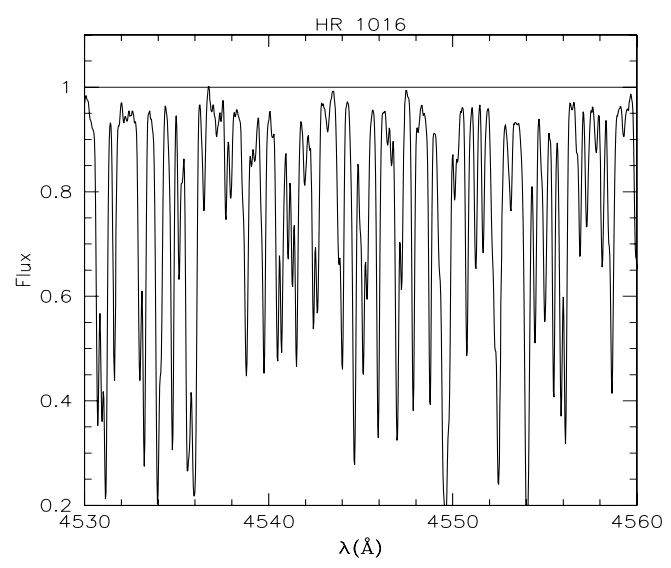

Fig. 1. An example of the normalization of the continuum in the bluest wavelength end used in this work.

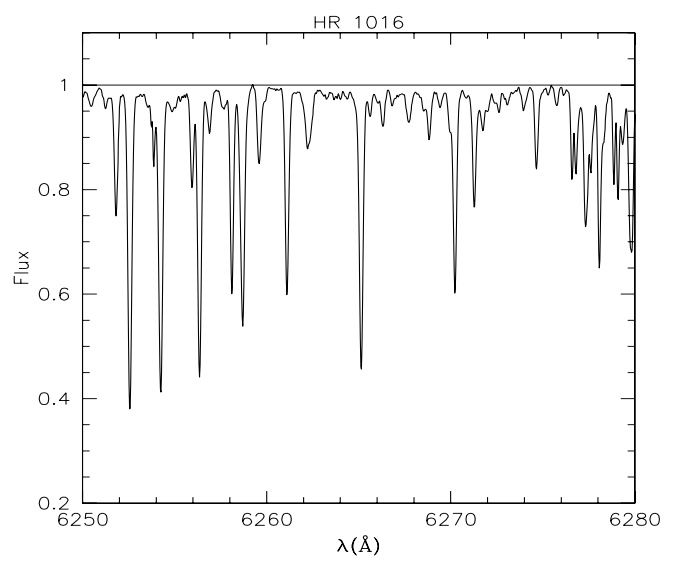

Fig. 2. A second example of the normalization of the continuum in a redder wavelength.

sections of the spectra. These sections were chosen to avoid spectral regions highly contaminated by telluric lines and to avoid the wings of very strong lines that lower the continuum level. The pseudo-continuum level was carefully determined by identifying regions apparently free of spectral lines with the help of a high resolution solar spectrum atlas (Kurucz et al. 1984). The continuum level was fitted with a low order Legendre polynomial crossing these regions. The crowding of the lines on the bluest end of the spectra makes this task more challenging than in the red end of the spectra. Nevertheless, we were able to find satisfactory fittings as exemplified in Figs. 1 and 2 for HR 1016.

For most of the elements we only used lines with $E W \mathrm{~s}$ smaller than $150 \mathrm{~m} \AA$. In this case the $E W$ s were determined by fitting Gaussian profiles to the observed ones with IRAF ${ }^{1}$.

This $E W$ value was determined to be the limit of saturation of the curve of growth. In this case the line growth is dominated by the Doppler broadening, which determines a Gaussian profile and leads to observed Gaussian profiles after convolution with the Gaussian instrumental broadening profile. The region of linear growth can be inferred in a plot of line depth vs. $E W$. We have empirically estimated the saturation limit by plotting the line depth vs. $E W$ for several lines of the same chemical species. This was first done for the Fe I lines in $\epsilon$ Vir, as shown in Fig. 3.

${ }^{1}$ IRAF is distributed by the National Optical Astronomy Observatory, which is operated by the Association of Universities for Research in Astronomy, Inc., under cooperative agreement with the National Science Foundation.

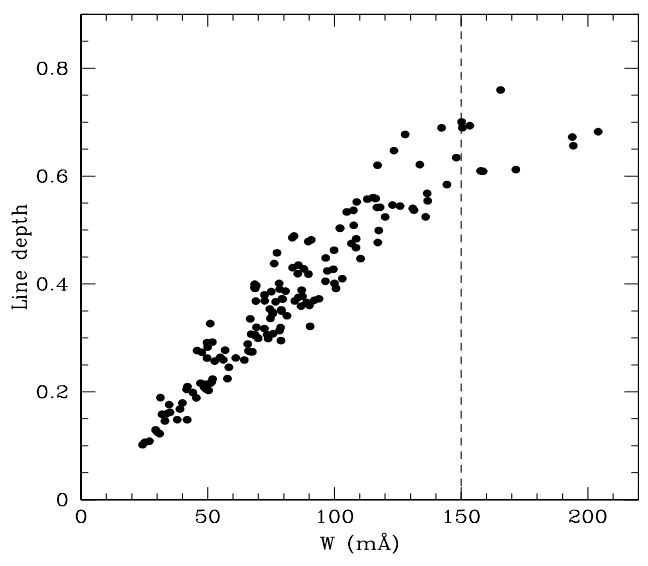

Fig. 3. Line depth vs. $E W$ for the Fe I lines of $\epsilon$ Vir. This plot was used to determine the $E W, 150 \mathrm{~m} \AA$, where saturation effects become important and a Gaussian profile is no longer the best approximation of the line profile.

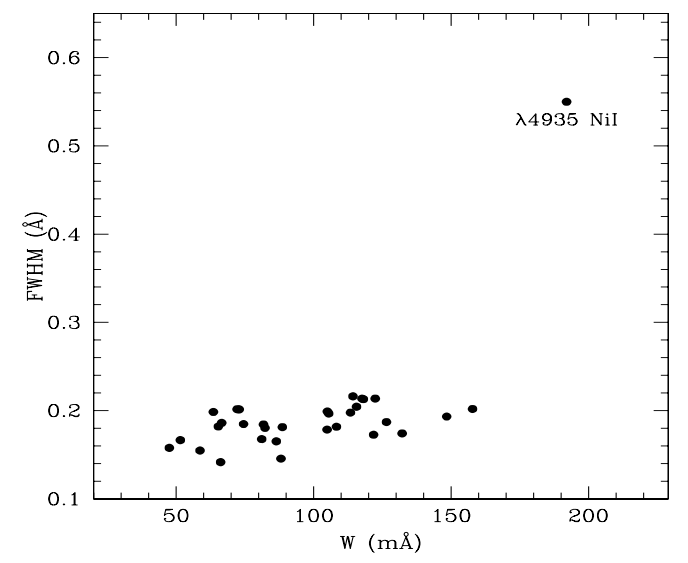

Fig. 4. Plot of FWHM vs. EW for the lines of HR 5058 in the $\lambda \lambda 4880-$ 5165 spectral range. This plot was done for all stars and used along with the plot of line depth vs. $E W$ to identify anomalous lines such as the NiI $\lambda 4935$ line seen in this plot. Lines departing from the expected trend were excluded from the analysis.

A significant departure from a linear growth happens only for $E W$ s larger than $\approx 150 \mathrm{~m} \AA$. Similar plots were made for all stars and this same limit proved reliable in all cases.

Lines stronger than $150 \mathrm{~m} \AA$ were used only for elements with only a few lines available throughout the spectra, namely $\mathrm{Na}, \mathrm{Mg}, \mathrm{Al}, \mathrm{Cu}, \mathrm{Zn}, \mathrm{Sr}, \mathrm{Y}, \mathrm{Zr}, \mathrm{Ba}, \mathrm{La}, \mathrm{Ce}$, and $\mathrm{Nd}$. In this case the $E W$ s were determined by fitting Voigt profiles to the observed ones. Slightly blended lines were measured using the deblending capabilities of IRAF. The $E W$ s of all the measured lines are listed in the appendix (Tables 8 and 9).

The plots of line depth vs. $E W$ were also used to test the reliability of the measurements, along with plots of the full width half maximum (FWHM) vs. $E W$. For the latter we expect the FWHM values to be distributed near the expected value for the spectral resolving power, and to slowly increase with increasing $E W$ due to its progressive departure from a purely Gaussian profile. Any line having, in any of these plots, a behavior differing from the general expected trend was excluded from the analysis. Figure 4 shows an example of the FWHM vs. $E W$ plot for the star HR 5058, where the NiI $\lambda 4935$ line is clearly seen to not be well-fitted by a Gaussian profile, this line then being excluded from the analysis of this star. 


\section{Atmospheric parameters}

\subsection{The differential analysis}

We conducted a model atmosphere analysis using the NMARCS grid (Plez et al. 1992; Edvardsson et al. 1993) originally developed by Gustafsson et al. (1975) and Bell et al. (1976). The models assume a plane parallel geometry, local thermodynamic equilibrium (LTE) and radiative equilibrium. We conducted a differential analysis using $\epsilon$ Vir (HD 113226) as a standard star. $\epsilon$ Vir has a solar like abundance pattern and is the same standard star used in the analysis by Zacs (1994).

Within a differential analysis a robust scale for comparison of stellar parameters is established for similar stars. As will be seen in the following discussion, our sample defines a rather small range in the atmospheric parameters space, a fact that fully supports our approach. In this sense, we can safely disregard uncertainties due to the choice of model atmospheres, convection, inhomogeneities and NLTE since we expect these effects to be similar among the sample stars.

The effective temperature ( $\left.T_{\text {eff }}\right)$ for $\epsilon$ Vir was calculated using the colors $(R-I)$ (Hoffleit \& Jaschek 1982) and $(V-K)$ (Johnson et al. 1966) with the metallicity independent relations by McWilliam (1990), for $(R-I)$ and $(V-K)$, and by Blackwell \& Lynas-Gray (1998), for $(V-K)$. A mean $T_{\text {eff }}$ was then calculated for the $(V-K)$ index, using the two calibrations, and this value was averaged with the $T_{\text {eff }}$ given by the $(R-I)$ index. The temperature thus obtained was $T_{\text {eff }}=5082 \mathrm{~K}$. In a differential analysis the exact values of the parameters are of much reduced importance, once the scales are homogeneous and internally consistent.

With the $V_{T}$ magnitude from Tycho (ESA 1997) and the bolometric correction from Landolt \& Börnstein (1982) we calculated the luminosity of $\epsilon$ Vir, $\log \left(L / L_{\odot}\right)=1.83$. The star was then placed in the HR diagram with evolutionary tracks from Schaller et al. (1992) and Schaerer et al. (1993). Assuming a solar metallicity a mass estimate was then interpolated, $M=2.80 M_{\odot}$. Finally, using the well known equation, $\log g=\log g_{\odot}+\log \left(M / M_{\odot}\right)+4 \log \left(\right.$ Tef $/$ Tef $\left.f_{\odot}\right)-\log \left(L / L_{\odot}\right)$, we calculated its surface gravity, $\log g=2.83$.

The microturbulence velocity $(\xi)$ was then determined spectroscopically, by requiring the abundance obtained from Fe I lines to have a null correlation with the $E W$. In this calculation we firstly adopted laboratory $g f \mathrm{~s}$ for $18 \mathrm{Fe}$ I lines given by Blackwell et al. (1995) and Holweger et al. (1995). The microturbulence velocity thus obtained was $\xi=1.86 \mathrm{~km} \mathrm{~s}^{-1}$.

By calculating the atmospheric parameters we determine a unique value for the metallicity. Using the above parameters a value of $[\mathrm{Fe} / \mathrm{H}]=+0.12 \pm 0.08$ was found. Since this value is different from the one assumed to calculate the stellar mass, we recalculated the parameters by adopting this new value for the metallicity. The new parameters thus calculated are $T_{\text {eff }}=5082 \mathrm{~K}, \log g=2.85 \mathrm{dex}, \xi=1.86 \mathrm{~km} \mathrm{~s}^{-1},[\mathrm{Fe} / \mathrm{H}]=$ $+0.12 \pm 0.08, \log \left(L / L_{\odot}\right)=1.83$ and $M=2.89 M_{\odot}$.

From the quoted uncertainties in the calibrations used to derive the $T_{\text {eff }}$, we estimate its $1 \sigma$ uncertainty to be $50 \mathrm{~K}$. The uncertainty in $\log g$ is estimated to be \pm 0.13 dex. This value is obtained by the propagation of the uncertainties related to the quantities used in its calculation; \pm 0.87 mas for $\pi, \pm 0.01$ mag for the BC, $\pm 0.002 \mathrm{mag}$ for $V_{T}, \pm 50 \mathrm{~K}$ for $T_{\text {eff }}, \pm 0.2 M_{\odot}$ for the mass, and assuming negligible uncertainties in the solar parameters. Note that the mass uncertainty is only related to the error bars in $\log \left(L / L_{\odot}\right)$ and $T_{\text {eff }}$, and does not take into account possible uncertainties inherent to the adopted tracks. Finally, the uncertainty in $\xi$ is estimated to be $\pm 0.08 \mathrm{~km} \mathrm{~s}^{-1}$, given by the uncertainty in the slope of the correlation of $[\mathrm{Fe} / \mathrm{H}]$ against $E W$.

This set of parameters for $\epsilon$ Vir is in good agreement with those previously determined in the literature, as can be noted by comparison with the values listed in the catalogue by Cayrel de Strobel et al. (2001). In particular we note the parameters obtained by McWilliam (1990), and listed in Table 1, $T_{\text {eff }}=$ $5060 \mathrm{~K}, \log g=2.97,[\mathrm{Fe} / \mathrm{H}]=+0.15$, and $\xi=1.90 \mathrm{~km} \mathrm{~s}^{-1}$.

Using the parameters we determined above and $E W \mathrm{~s}$ from the spectrum of $\epsilon$ Vir, we derived a new set of $g f \mathrm{~s}$, for the whole set of lines employed in this work, by requiring its abundance pattern to be solar (where we adopt the solar abundances from Anders \& Grevesse 1989). The set of $g f$ s thus calculated was used to derive the atmospheric parameters and abundances for the other stars. The $g f \mathrm{~s}$ are listed in the appendix (Tables 8 and 9) along with the EWs.

\subsection{Atmospheric parameters for the sample stars}

For the other sample stars, $T_{\text {eff }}$ was calculated by requiring a null correlation of the iron abundance as given by the Fe I lines with the excitation potential $(\chi)$, thus fulfilling the excitation equilibrium. The surface gravity is found by requiring both Fe I and Fe II lines to have the same mean abundance, thus fulfilling the ionization equilibrium. The microturbulence velocity is found by requiring the Fe I abundance to have a null correlation with the equivalent widths. By simultaneously constraining these parameters we also determine the metallicity, $[\mathrm{Fe} / \mathrm{H}]$. The parameters thus obtained are listed in Table 2. It can be seen that the stars define a narrow range of $T_{\mathrm{eff}}, \log g$ and $[\mathrm{Fe} / \mathrm{H}]$.

Figures 5 and 6 show examples of the null correlation obtained between the iron abundance and the excitation potential or the $E W$ s, respectively. In Fig. 5 we also show the Fe II lines to exemplify the ionization equilibrium.

\subsection{Uncertainties of the atmospheric parameters}

The uncertainties of the atmospheric parameters were calculated for a representative star, HR 2392, which has atmospheric parameters lying close to the center of the range defined by the whole sample.

When determining the parameters, namely $T_{\text {eff }}$ and $\xi$, from the Fe I lines, we search for a linear fit where the angular coefficient is statistically null. The uncertainty of this coefficient sets the uncertainty of the $T_{\text {eff }}$ and $\xi$ determinations. To find the $1 \sigma$ uncertainty of these parameters we change their values, respectively, in the Fe I abundance vs. line excitation potential, and the Fe I abundance vs. EW diagrams, until the angular coefficients of the linear fit match their own uncertainty.

In order to find the $1 \sigma$ uncertainty of the surface gravity a different approach is required. The mean Fe abundances as given from the Fe I lines and from the Fe II lines have, in general, different standard deviations. The gravity is then changed until the difference between the mean abundances from Fe I and Fe II equals the larger of the standard deviations. This change in the gravity is considered to be the $1 \sigma$ uncertainty in $\log g$. The uncertainties thus calculated are listed in Table 3.

We also estimated the uncertainty of the measurements of the EWs using two stars with very similar atmospheric parameters, HR 649 and HR 1016. For the lines measured with Gaussian profiles, under the hypothesis that the two stars should have the same set of atmospheric parameters, and thus EWs, any difference on the $E W$ s are due only to errors on the measurements. It is 
Table 2. The atmospheric parameters, effective temperature $\left(T_{\text {eff }}\right)$, surface gravity $(\log g)$, microturbulence velocity $(\xi)$ and metallicity $([\mathrm{Fe} / \mathrm{H}])$ (we use throughout the notation $[\mathrm{A} / \mathrm{B}]=\log \left(N(\mathrm{~A}) / N(\mathrm{~B})_{\mathrm{star}}-\log \left(N(\mathrm{~A}) / N(\mathrm{~B})_{\odot}\right)\right.$, derived for the sample stars as described in the text. The values for $[\mathrm{Fe} \mathrm{I} / \mathrm{H}]$ and $[\mathrm{Fe} \mathrm{II} / \mathrm{H}]$ are followed by the standard deviation and the number of lines on which the abundance is based.

\begin{tabular}{cccccc}
\hline \hline Star & $T_{\text {eff }}(\mathrm{K})$ & $\log g$ & $\xi\left(\mathrm{km} \mathrm{s}^{-1}\right)$ & {$[\mathrm{Fe} \mathrm{I} / \mathrm{H}] \pm \sigma(\#)$} & {$[\mathrm{Fe} \mathrm{II} / \mathrm{H}] \pm \sigma(\#)$} \\
\hline HR 440 & 4780 & 2.43 & 1.71 & $-0.34 \pm 0.07(129)$ & $-0.33 \pm 0.07(13)$ \\
HR 649 & 5120 & 2.49 & 1.96 & $-0.14 \pm 0.06(117)$ & $-0.15 \pm 0.07(13)$ \\
HR 1016 & 5080 & 2.60 & 1.80 & $-0.11 \pm 0.06(119)$ & $-0.11 \pm 0.09(13)$ \\
HR 1326 & 4650 & 2.51 & 1.52 & $+0.00 \pm 0.07(119)$ & $+0.01 \pm 0.16(13)$ \\
HR 2392 & 4940 & 2.65 & 1.87 & $-0.09 \pm 0.12(115)$ & $-0.08 \pm 0.15(12)$ \\
HR 4608 & 4920 & 2.58 & 1.71 & $-0.35 \pm 0.05(117)$ & $-0.34 \pm 0.06(13)$ \\
$\epsilon$ Vir & 5082 & 2.85 & 1.86 & +0.12 & +0.12 \\
HR 5058 & 4790 & 2.67 & 1.97 & $-0.12 \pm 0.13(109)$ & $-0.13 \pm 0.22(11)$ \\
HR 5802 & 5010 & 2.89 & 1.67 & $-0.02 \pm 0.06(129)$ & $-0.03 \pm 0.06(13)$ \\
HR 7321 & 4810 & 2.48 & 1.70 & $-0.19 \pm 0.06(125)$ & $-0.17 \pm 0.07(13)$ \\
HR 8115 & 4910 & 2.41 & 1.85 & $-0.04 \pm 0.07(125)$ & $-0.03 \pm 0.09(13)$ \\
HR 8204 & 5250 & 1.53 & 2.49 & $-0.09 \pm 0.12(105)$ & $-0.13 \pm 0.11(10)$ \\
HD 205011 & 4780 & 2.41 & 1.70 & $-0.14 \pm 0.09(117)$ & $-0.13 \pm 0.10(13)$ \\
HR 8878 & 4370 & 1.91 & 1.61 & $-0.67 \pm 0.07(131)$ & $-0.63 \pm 0.10(13)$ \\
\hline
\end{tabular}

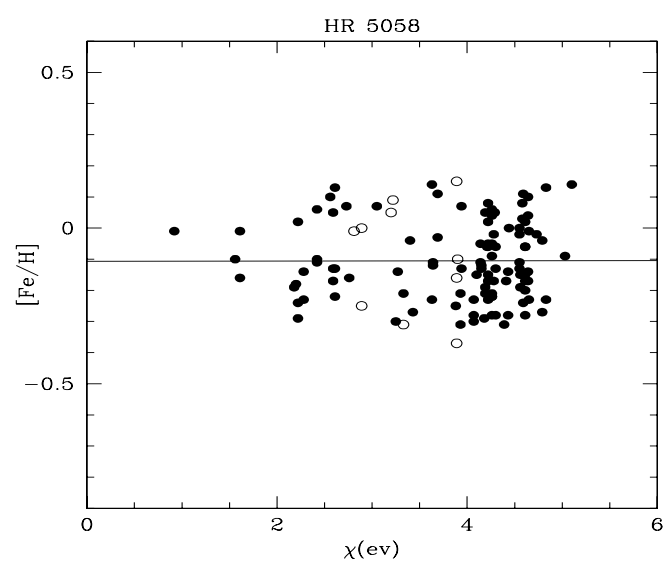

Fig. 5. Iron abundance of both Fe I (full circles) and Fe II lines (open circles) vs. the line excitation potential for the star HR 5058. The solid line is a linear fit to the Fe I lines indicating that the excitation equilibrium was fulfilled. The ionization equilibrium was also obtained by setting the Fe I and Fe II abundances to be equal, determining the surface gravity.

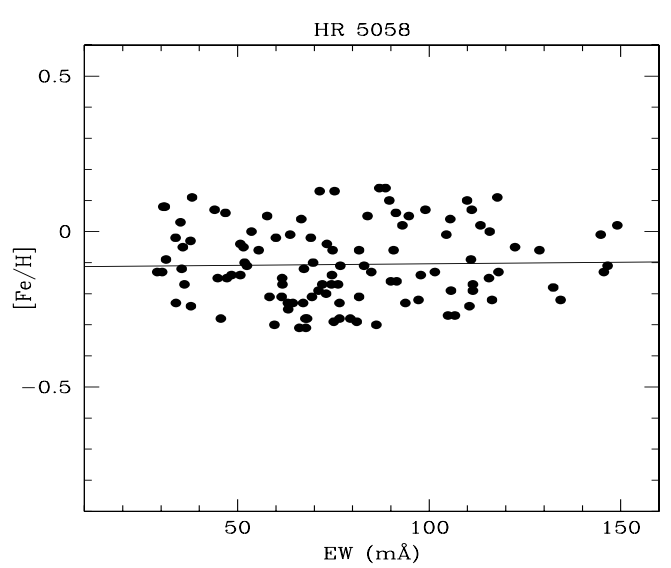

Fig. 6. Iron abundance vs. $E W$ for the Fe I lines of HR 5058. This plot was used to determine the microturbulence velocity by requiring a null correlation between $[\mathrm{Fe} / \mathrm{H}]$ and the $E W \mathrm{~s}$.

clear that such a hypothesis tends to overestimate the uncertainty since actual differences between the two sets of $E W$ s are expected. However, the uncertainty thus calculated is only $\pm 3 \mathrm{~m} \AA$,
Table 3. Uncertainties of the atmospheric parameters and of the equivalent widths.

\begin{tabular}{cccc}
\hline \hline Parameter & $\sigma$ & Range of $E W$ & $\sigma$ \\
\hline$T_{\text {eff }}(\mathrm{K})$ & \pm 50 & $E W<150 \mathrm{~m} \AA$ & $\pm 3 \mathrm{~m} \AA$ \\
$\log g($ dex $)$ & \pm 0.35 & $150 \mathrm{~m} \AA<E W<400 \mathrm{~m} \AA$ & $\pm 10 \mathrm{~m} \AA$ \\
$\xi\left(\mathrm{km} \mathrm{s}^{-1}\right)$ & \pm 0.06 & $E W>400 \mathrm{~m} \AA$ & $\pm 20 \mathrm{~m} \AA$ \\
\hline
\end{tabular}

which emphasizes the quality of our data and the good internal consistency of the measurements.

For the stronger lines measured with Voigt profiles we adopted a different approach, clearly needed since most of these lines are due to s-process elements, and thus expected to have different intensities in the chosen stars. In this case, the uncertainty was estimated by fitting each line a few times, at each time slightly changing the limits in wavelength for the profile fitting, keeping control of the FWHM of the line Gaussian core. In this sense we can verify the robustness of the fitting routine and the sensibility of the measurements on the adopted wavelength limits in a statistically significant way. In this case the uncertainties found were $\pm 10 \mathrm{~m} \AA$ for $150 \mathrm{~m} \AA<E W<400 \mathrm{~m} \AA$ and $\pm 20 \mathrm{~m} \AA$ for $E W>400 \mathrm{~m} \AA$.

\section{Abundances, masses and ages}

The abundances were calculated based on the $E W \mathrm{~s}$ and the $g f \mathrm{~s}$ previously derived. For the elements $\mathrm{Mg}, \mathrm{Sc}, \mathrm{V}, \mathrm{Mn}, \mathrm{Co}$ and $\mathrm{Cu}$ we considered the hyperfine structure following Steffen (1985). The abundances are listed in Table 4. We discuss below the uncertainties of the abundances and then the abundance pattern of each star in comparison with previous analyses available in the literature. In the Figs. 7 to 9, the error bar on the abundances is always the larger value between the standard deviation of the $[\mathrm{X} / \mathrm{Fe}]$ abundance ratios given by the stellar lines (listed in Table 4), and the theoretically calculated uncertainties for each element, based on the atmospheric parameter and $E W$ uncertainties derived in Sect. 4.1. These theoretical error calculations are listed in Table 5. 
Table 4. Mean elemental abundances for the program stars, followed by the standard deviation, when applicable, and the number of lines on which the abundance is based.

\begin{tabular}{|c|c|c|c|c|c|c|}
\hline$[\mathrm{X} / \mathrm{Fe}]$ & HR 440 & HR 649 & HR 1016 & HR 1326 & HR 2392 & HR 4608 \\
\hline $\mathrm{Na}$ & $-0.28(02)$ & $-0.09(02)$ & $-0.17(02)$ & $-0.31(02)$ & $-0.15(02)$ & $-0.26(02)$ \\
\hline $\mathrm{Mg}$ & $+0.02 \pm 0.07(03)$ & $-0.05 \pm 0.08(04)$ & $-0.07 \pm 0.05(04)$ & $-0.07 \pm 0.01(03)$ & $+0.15 \pm 0.19(04)$ & $-0.04 \pm 0.05(04)$ \\
\hline $\mathrm{Al}$ & $+0.04(02)$ & $-0.09(02)$ & $-0.12(02)$ & $+0.02(02)$ & $+0.03(02)$ & $+0.01(02)$ \\
\hline $\mathrm{Si}$ & $+0.01 \pm 0.07(15)$ & $-0.10 \pm 0.08$ & $-0.12 \pm 0.07(14)$ & $-0.09 \pm 0.09(10)$ & $0.00 \pm 0.12(08)$ & $-0.07 \pm 0.04(10)$ \\
\hline $\mathrm{Ca}$ & $-0.09 \pm 0.08(12)$ & $-0.06 \pm 0.08$ & $-0.07 \pm 0.03(09)$ & $-0.15 \pm 0.08$ & $-0.07 \pm 0.08(10)$ & $-0.03 \pm 0.06(11)$ \\
\hline Sc & $-0.03 \pm 0.09(07)$ & $-0.08 \pm 0.09(07)$ & $-0.13 \pm 0.08(07)$ & $-0.18 \pm 0.08(07)$ & $-0.03 \pm 0.08(07)$ & $-0.02 \pm 0.04(07)$ \\
\hline $\mathrm{Ti}$ & $0.00 \pm 0.06(30)$ & $-0.02 \pm 0.07(34)$ & $-0.08 \pm 0.06(35)$ & $-0.02 \pm 0.08$ & $-0.02 \pm 0.14(30)$ & $+0.01 \pm 0.08(36)$ \\
\hline V & $-0.08 \pm 0.06(11)$ & $-0.06 \pm 0.09$ & $-0.16 \pm 0.05(11)$ & $-0.05 \pm 0.18(10)$ & $-0.03 \pm 0.07(10)$ & $-0.11 \pm 0.05(09)$ \\
\hline $\mathrm{Cr}$ & $-0.10 \pm 0.05(25)$ & $-0.07 \pm 0.08(27)$ & $-0.09 \pm 0.07(24)$ & $-0.11 \pm 0.08(22)$ & $-0.09 \pm 0.17(16)$ & $-0.10 \pm 0.06(22)$ \\
\hline Mn & $-0.18 \pm 0.05(06)$ & $-0.20 \pm 0.07(06)$ & $-0.20 \pm 0.06(06)$ & $-0.12 \pm 0.09(04)$ & $-0.09 \pm 0.12(05)$ & $-0.24 \pm 0.13(06)$ \\
\hline $\mathrm{Co}$ & $+0.02 \pm 0.06(09)$ & $-0.02 \pm 0.06(09)$ & $-0.09 \pm 0.10(10)$ & $-0.12 \pm 0.07(10)$ & $-0.01 \pm 0.09(08)$ & $0.00 \pm 0.06(09)$ \\
\hline $\mathrm{Ni}$ & $-0.09 \pm 0.06(26)$ & $-0.16 \pm 0.06(28)$ & $-0.16 \pm 0.07(27)$ & $-0.06 \pm 0.10(25)$ & $-0.13 \pm 0.13(24)$ & $-0.11 \pm 0.05(24)$ \\
\hline $\mathrm{Cu}$ & $-0.06 \pm 0.17(03)$ & $-0.17 \pm 0.26(03)$ & $-0.09(02)$ & $-0.03 \pm 0.10(03)$ & $-0.04(02)$ & $-0.01 \pm 0.42(03)$ \\
\hline $\mathrm{Zn}$ & $+0.09(01)$ & $+0.05(01)$ & $+0.05(01)$ & $-0.10(01)$ & $-0.07(01)$ & $+0.04(01)$ \\
\hline $\mathrm{Sr}$ & $-0.16(01)$ & $+0.16(01)$ & $+0.12(01)$ & $+0.03(01)$ & $+1.24(01)$ & $+0.60(01)$ \\
\hline $\mathrm{Y}$ & $-0.23 \pm 0.09(05)$ & $+0.01 \pm 0.09(06)$ & $-0.11 \pm 0.04(06)$ & $-0.06 \pm 0.09(06)$ & $+1.23 \pm 0.36(06)$ & $+0.44 \pm 0.10(06)$ \\
\hline $\mathrm{Zr}$ & $-0.21 \pm 0.06(03)$ & $-0.04 \pm 0.25(03)$ & $-0.08 \pm 0.09(03)$ & $-0.07 \pm 0.11(03)$ & $+1.04 \pm 0.46(03)$ & $+0.60(02)$ \\
\hline $\mathrm{Ba}$ & $-0.28 \pm 0.13(03)$ & $+0.08 \pm 0.17(03)$ & $0.00 \pm 0.12(03)$ & $-0.27 \pm 0.16(03)$ & $+1.17 \pm 0.44(03)$ & $+0.54 \pm 0.31(03)$ \\
\hline $\mathrm{La}$ & $-0.14 \pm 0.16(04)$ & $+0.05 \pm 0.20(03)$ & $-0.06 \pm 0.14(04)$ & $-0.09 \pm 0.20(04)$ & $+1.52 \pm 0.34(04)$ & $+0.57 \pm 0.08(04)$ \\
\hline $\mathrm{Ce}$ & $-0.10 \pm 0.06(05)$ & $+0.09 \pm 0.10(05)$ & $+0.05 \pm 0.10(05)$ & $+0.06 \pm 0.15(05)$ & $+1.48 \pm 0.55(05)$ & $+0.68 \pm 0.25(05)$ \\
\hline $\mathrm{Nd}$ & $-0.08(02)$ & $+0.06(02)$ & $+0.02(02)$ & $+0.06(02)$ & $+0.91(02)$ & $+0.58(02)$ \\
\hline $\mathrm{Sm}$ & $-0.19(01)$ & $-0.13(01)$ & $-0.12(01)$ & $-0.06(01)$ & $+0.78(01)$ & $+0.20(01)$ \\
\hline $\mathrm{Eu}$ & - & - & $-0.01(01)$ & $+0.04(01)$ & $+0.53(01)$ & $+0.24(01)$ \\
\hline $\mathrm{Gd}$ & $-0.32(01)$ & - & $-0.09(01)$ & $-0.17(01)$ & $+0.06(01))$ & - \\
\hline
\end{tabular}

Table 4. continued.

\begin{tabular}{|c|c|c|c|c|c|c|}
\hline$\overline{[\mathrm{X} / \mathrm{Fe}]}$ & HR 5058 & HR 5802 & HR 7321 & HR 8115 & HR 8204 & HR 8878 \\
\hline $\mathrm{Na}$ & $-0.10(02)$ & $-0.17(02)$ & $-0.28(02)$ & $-0.05(02)$ & $-0.21(02)$ & $-0.21(2)$ \\
\hline $\mathrm{Mg}$ & $-0.01 \pm 0.16(4)$ & $-0.03 \pm 0.07(04)$ & $-0.05 \pm 0.06(04)$ & $-0.10 \pm 0.04(04)$ & $+0.04 \pm 0.21(04)$ & $+0.19 \pm 0.09(03)$ \\
\hline $\mathrm{Al}$ & $+0.05(02)$ & $-0.03(02)$ & $-0.07(02)$ & $-0.07(02)$ & $+0.33(01)$ & $+0.21(01)$ \\
\hline $\mathrm{Si}$ & $+0.03 \pm 0.13$ & $-0.07 \pm 0.05(11)$ & $-0.02 \pm 0.08(15)$ & $-0.05 \pm 0.09$ & $-0.02 \pm 0.15(11)$ & $+0.16 \pm 0.04(12)$ \\
\hline $\mathrm{Ca}$ & $-0.10 \pm 0.14(08)$ & $-0.09 \pm 0.03(10)$ & $-0.10 \pm 0.06(12)$ & $-0.08 \pm 0.06(11)$ & $-0.05 \pm 0.11(07)$ & $+0.10 \pm 0.05(11)$ \\
\hline $\mathrm{Sc}$ & $-0.02 \pm 0.08(07)$ & $-0.07 \pm 0.05(07)$ & $-0.13 \pm 0.07(07)$ & $-0.18 \pm 0.09(07)$ & $-0.33 \pm 0.08(05)$ & $+0.05 \pm 0.06(07)$ \\
\hline $\mathrm{Ti}$ & $-0.02 \pm 0.13(32)$ & $-0.06 \pm 0.07(35)$ & $-0.06 \pm 0.06(34)$ & $-0.12 \pm 0.07(36)$ & $0.00 \pm 0.18(29)$ & $+0.34 \pm 0.13(30)$ \\
\hline V & $+0.08 \pm 0.11(10)$ & $-0.06 \pm 0.04(10)$ & $-0.15 \pm 0.02$ & $-0.21 \pm 0.05(11)$ & $-0.13 \pm 0.12(09)$ & $+0.23 \pm 0.17(11)$ \\
\hline $\mathrm{Cr}$ & $+0.04 \pm 0.28(21)$ & $-0.03 \pm 0.09(25)$ & $-0.06 \pm 0.11(26)$ & $+0.17 \pm 0.12(27)$ & $-0.11 \pm 0.18$ & $-0.05 \pm 0.08(23)$ \\
\hline Mn & $-0.13 \pm 0.09(04)$ & $-0.17 \pm 0.08(06)$ & $-0.18 \pm 0.04(05)$ & $-0.23 \pm 0.08(06)$ & $-0.16 \pm 0.32(07)$ & $-0.24 \pm 0.05(06)$ \\
\hline $\mathrm{Co}$ & $0.00 \pm 0.05(08)$ & $-0.08 \pm 0.06(10)$ & $-0.07 \pm 0.04(09)$ & $-0.12 \pm 0.08(10)$ & $-0.03 \pm 0.22(08)$ & $+0.07 \pm 0.07(07)$ \\
\hline $\mathrm{Ni}$ & $-0.08 \pm 0.14(23)$ & $-0.13 \pm 0.03(21)$ & $-0.12 \pm 0.05(26)$ & $-0.16 \pm 0.05(22)$ & $-0.20 \pm 0.10(22)$ & $-0.06 \pm 0.07(24)$ \\
\hline $\mathrm{Cu}$ & $+0.55(02)$ & $-0.03 \pm 0.05(03)$ & $+0.06 \pm 0.09(03)$ & $+0.12 \pm 0.13(03)$ & $-0.03(02)$ & $+0.19(02)$ \\
\hline $\mathrm{Zn}$ & $-0.21(01)$ & $-0.04(01)$ & $0.00(01)$ & $-0.03(01)$ & $+0.18(01)$ & $+0.14(01)$ \\
\hline $\mathrm{Sr}$ & $+1.38(01)$ & $+0.70(01)$ & $+0.51(01)$ & $+0.49(01)$ & $+2.21(01)$ & $+0.08(01)$ \\
\hline $\mathrm{Y}$ & $+0.99 \pm 0.33(06)$ & $+0.50 \pm 0.11(06)$ & $+0.29 \pm 0.12(06)$ & $+0.37 \pm 0.10(06)$ & $+1.66 \pm 0.45(05)$ & $-0.02 \pm 0.13(05)$ \\
\hline $\mathrm{Zr}$ & $+0.83 \pm 0.36(03)$ & $+0.48 \pm 0.04(03)$ & $+0.27 \pm 0.04(03)$ & $+0.22 \pm 0.04(03)$ & $+1.00 \pm 0.60(03)$ & $-0.07 \pm 0.15(03)$ \\
\hline $\mathrm{Ba}$ & $+0.93 \pm 0.36(03)$ & $+0.16 \pm 0.17(03)$ & $+0.31 \pm 0.39(03)$ & $+0.31 \pm 0.26(03)$ & $+1.08 \pm 0.39(03)$ & $-0.38 \pm 0.14(03)$ \\
\hline $\mathrm{La}$ & $+1.28 \pm 0.43(04)$ & $+0.27 \pm 0.10(04)$ & $+0.23 \pm 0.14(04)$ & $+0.19 \pm 0.18(04)$ & $+1.32 \pm 0.57(04)$ & $-0.12 \pm 0.07(04)$ \\
\hline $\mathrm{Ce}$ & $+1.05 \pm 0.53(05)$ & $+0.20 \pm 0.10(04)$ & $+0.32 \pm 0.17(05)$ & $+0.23 \pm 0.16(05)$ & $+1.48 \pm 0.71(05)$ & $+0.05 \pm 0.20(05)$ \\
\hline $\mathrm{Nd}$ & $+0.78(02)$ & $+0.12(02)$ & $+0.15(02)$ & $+0.11(02)$ & $+1.01(02)$ & $+0.10(02)$ \\
\hline $\mathrm{Sm}$ & $+0.56(01)$ & $0.00(01)$ & $+0.01(01)$ & $-0.11(01)$ & $+0.16(01)$ & $-0.07(01)$ \\
\hline $\mathrm{Eu}$ & $+0.49(01)$ & $0.00(01)$ & - & $-0.03(01)$ & $+0.02(01)$ & $+0.24(01)$ \\
\hline Gd & $-0.09(01)$ & - & $-0.24(01)$ & $-0.23(01)$ & - & $-0.29(01)$ \\
\hline
\end{tabular}

\subsection{Uncertainties of the abundances}

The abundances are subject to uncertainties coming from the determination of the atmospheric parameters. In order to estimate these uncertainties we change each atmospheric parameter by its own error, keeping the other ones with the original adopted values, and recalculate the abundances. In this way we measure the effect of each parameter uncertainty in the abundances. We also estimated the uncertainty in the abundances coming from the errors in the measurement of the EWs. These effects are listed in
Table 5. The calculations were done, again, for the star HR 2392. Assuming that the effects of the uncertainties of the parameters are independent, we can estimate a lower bound of the total uncertainty with Eq. (1). The total compounded uncertainty is also listed in Table 5.

$$
\sigma_{\text {total }}=\sqrt{\left(\sigma_{T_{\text {eff }}}\right)^{2}+\left(\sigma_{\log g}\right)^{2}+\left(\sigma_{\xi}\right)^{2}+\left(\sigma_{[\mathrm{Fe} / \mathrm{H}]}\right)^{2}+\left(\sigma_{W}\right)^{2}}
$$

In general each error source affects the abundances by less than 0.1 dex, except for the uncertainty introduced by $\log g$ in 


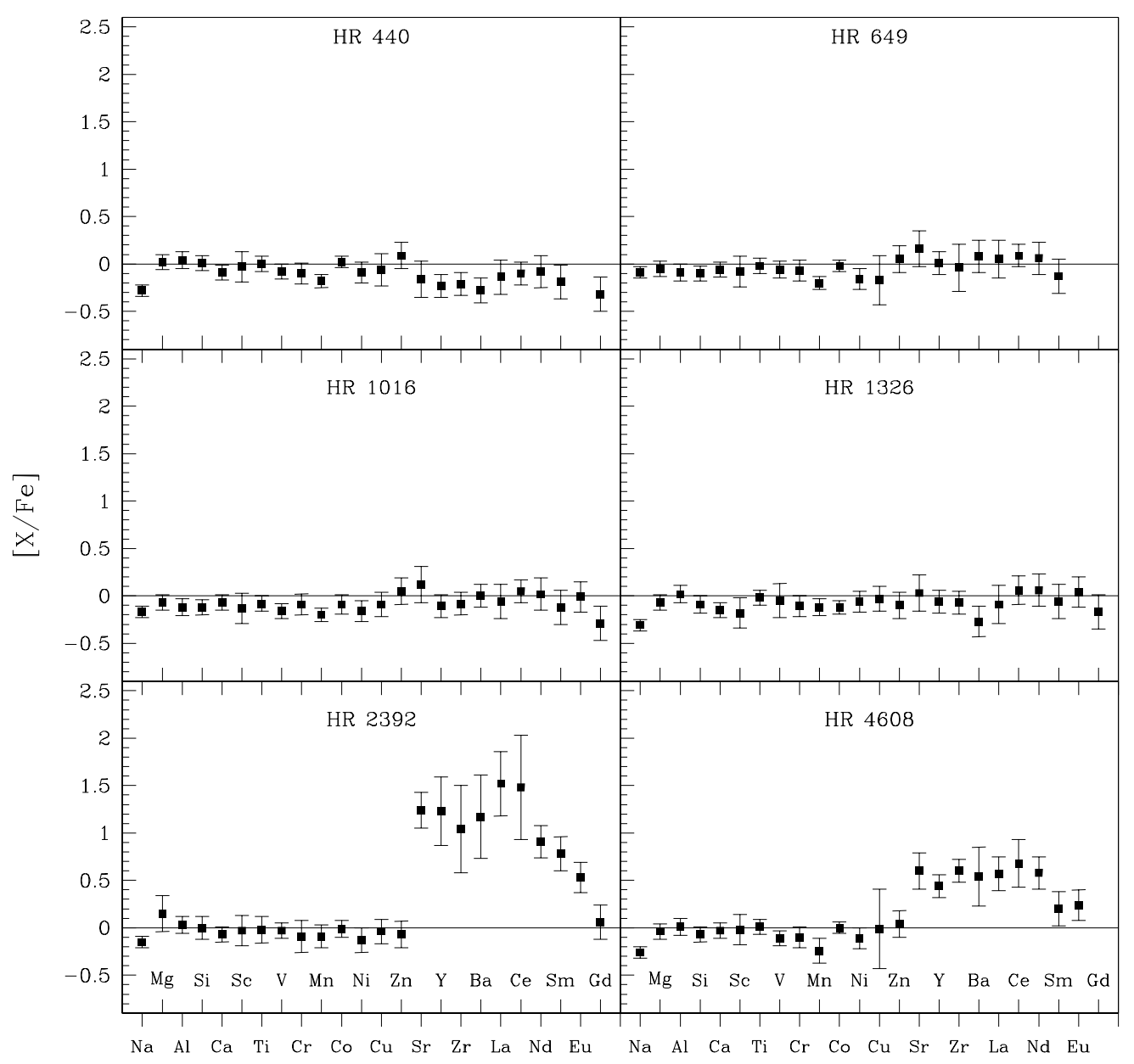

Fig. 7. The abundance pattern for HR 440, HR 649, HR 1016, HR 1326, HR 2392 and HR 4608.

Table 4. continued.

\begin{tabular}{lc}
\hline \hline$[\mathrm{X} / \mathrm{Fe}]$ & $\mathrm{HD} 205011$ \\
\hline $\mathrm{Na}$ & $-0.21(02)$ \\
$\mathrm{Mg}$ & $-0.08 \pm 0.04(03)$ \\
$\mathrm{Al}$ & $+0.01(02)$ \\
$\mathrm{Si}$ & $+0.05 \pm 0.09(11)$ \\
$\mathrm{Ca}$ & $-0.12 \pm 0.06(11)$ \\
$\mathrm{Sc}$ & $-0.15 \pm 0.10(07)$ \\
$\mathrm{Ti}$ & $-0.12 \pm 0.09(33)$ \\
$\mathrm{V}$ & $-0.18 \pm 0.04(11)$ \\
$\mathrm{Cr}$ & $-0.03 \pm 0.20(25)$ \\
$\mathrm{Mn}$ & $-0.21 \pm 0.09(07)$ \\
$\mathrm{Co}$ & $-0.12 \pm 0.09(10)$ \\
$\mathrm{Ni}$ & $-0.15 \pm 0.05(25)$ \\
$\mathrm{Cu}$ & $+0.12 \pm 0.14(03)$ \\
$\mathrm{Zn}$ & $-0.02(01)$ \\
$\mathrm{Sr}$ & $+0.89(01)$ \\
$\mathrm{Y}$ & $+0.90 \pm 0.32(05)$ \\
$\mathrm{Zr}$ & $+0.57 \pm 0.16(03)$ \\
$\mathrm{Ba}$ & $+0.66 \pm 0.34(03)$ \\
$\mathrm{La}$ & $+0.62 \pm 0.20(04)$ \\
$\mathrm{Ce}$ & $+0.63 \pm 0.33(05)$ \\
$\mathrm{Nd}$ & $+0.34(02)$ \\
$\mathrm{Sm}$ & $+0.09(01)$ \\
$\mathrm{Eu}$ & $+0.13(01)$ \\
$\mathrm{Gd}$ & $-0.13(01)$ \\
\hline &
\end{tabular}

the abundances derived from lines of singly ionized species. For this reason, the uncertainty of abundances derived solely from neutral species are usually smaller. The mean value of the theoretically estimated $\sigma_{\text {total }}$ is close to $0.12 \mathrm{dex}$, and does not exceed 0.20 dex.

However, the abundance uncertainties, as estimated by the dispersion of the mean of individual line abundances, of the stars with the largest excesses of s-process elements, sometimes appreciably surpass the values of Table 5. This is the case of HR 2392, 5058 and 8204, with the elements Y, Zr, La and Ce having dispersions of the mean reaching $\approx 0.6$ dex. This clearly reflects enhanced errors in measuring the very strong lines of these elements, leading us to the conclusion that at least for a few cases the abundance uncertainties given in Table 5 may be underestimated. The larger uncertainties in the abundances of these elements in these stars, notwithstanding, do not affect appreciably any of the conclusions of our analysis, specially the classification of these objects as mild or classical barium stars.

\subsection{Abundances}

HR 440

HR 440 is one of the selected normal giants. Its metallicity is lower than solar, $[\mathrm{Fe} / \mathrm{H}]=-0.34 \mathrm{dex}$. Its abundance pattern is shown in Fig. 7. We note it to be almost solar, the only significant difference is a $2 \sigma$ level deficiency for $\mathrm{Na}$. We also note indications of small deficiencies of $\mathrm{Mn}, \mathrm{Y}, \mathrm{Zr}, \mathrm{Ba}$ and $\mathrm{Gd}$ that are not significant within $2 \sigma$. 


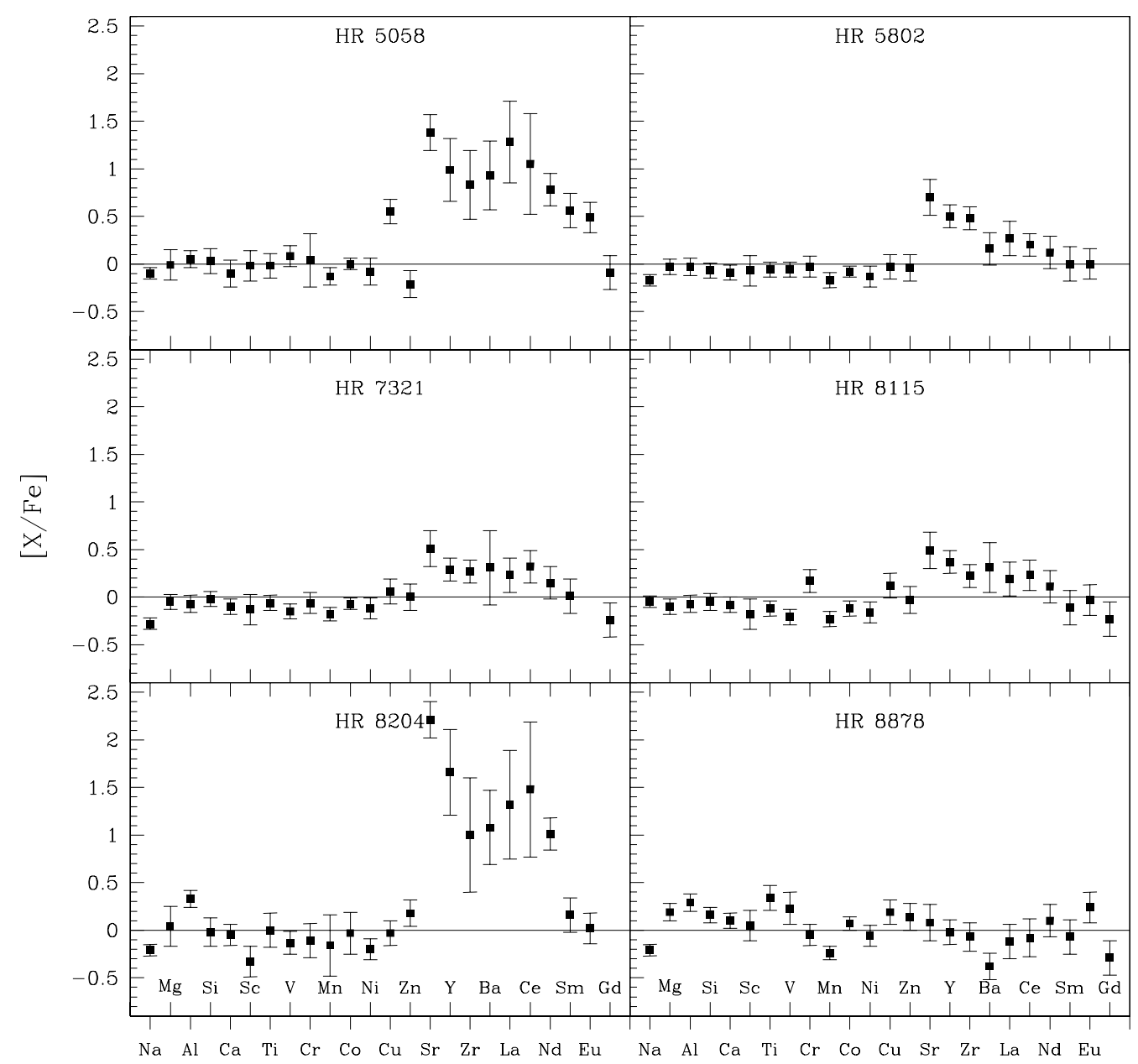

Fig. 8. The abundance pattern for HR 5058, HR 5802, HR 7321, HR 8115, HR 8204 and HR 8878.

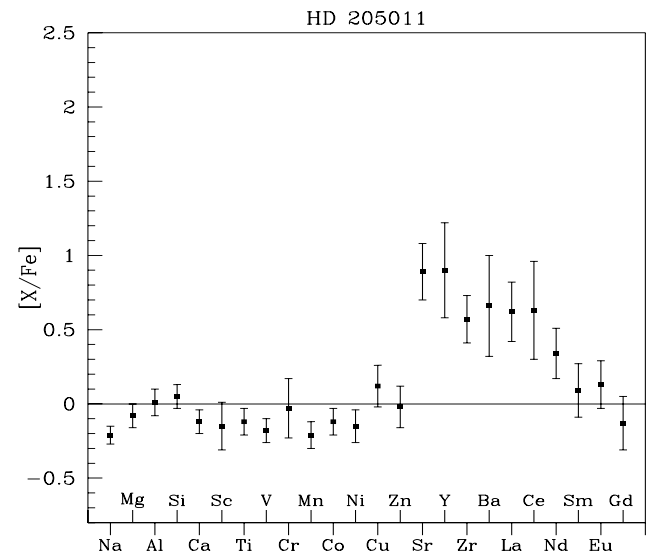

Fig. 9. The abundance pattern for HD 205011.

\section{HR 649}

HR 649 has a metallicity slightly lower than solar, $[\mathrm{Fe} / \mathrm{H}]=$ -0.14 dex, and is considered in the literature to be a mild barium star. However, its abundance pattern, shown in Fig. 7, is solar except for a $2 \sigma$-level deficiency of $\mathrm{Mn}$.

In the catalogue by $\mathrm{Lu}(1991)$ it has an index Ba0.3. Barium stars are classified according to their level of overabundance in the range $\mathrm{Ba} 0.1-5$, in a scheme first proposed by Warner (1965) and further extended (Keenan \& Pitts 1980), where Ba5 indicates the largest overabundances. Stars with Ba index less than $\mathrm{Ba} 2$ are the so-called mild barium stars.

This star is a confirmed binary system with a white dwarf companion identified by Böhm-Vitense \& Johnson (1985). It is thus one of the stars thought to support the scenario to explain the origin of the abundances in barium and mild barium stars.

It was previously analyzed by Zacs (1994), who found Y, Ba, and La to be overabundant and $\mathrm{Zr}$ and $\mathrm{Nd}$ to be normal. Except for $\mathrm{Zr}$ and $\mathrm{Nd}$ his results are based on fewer lines than ours. We also found $\mathrm{Sr}$ and $\mathrm{Ce}$ to be normal. Our results rely on higher $S / N$ data and more sophisticated methods to measure equivalent widths than those of Zacs (1994). Thus we conclude HR 649 not to be a mild barium star but a normal giant.

\section{HR 1016}

HR 1016 also has a metallicity slightly lower than solar, $[\mathrm{Fe} / \mathrm{H}]=-0.11 \mathrm{dex}$, and is also considered in the literature to be a mild barium star. It was first analyzed by Pilachowski (1977) who found small s-process enhancements for $\mathrm{Y}, \mathrm{Zr}$, and $\mathrm{Ce}$, and normal abundances for $\mathrm{Sr}, \mathrm{Ba}, \mathrm{La}, \mathrm{Pr}$, and $\mathrm{Nd}$. We found no record of a radial velocity variability or white dwarf companion in the literature. Our abundance pattern for this star is shown in Fig. 7. We found no indication of anomalous abundances of any s-process element. Its only peculiarities are deficiencies at the $2 \sigma$ level of $\mathrm{Na}$ and $\mathrm{Mn}$ (as in HR 649), and probably of V. We thus conclude this to be a case similar to that of HR 649: a normal giant misclassified as a mild barium star. 
Table 5. The uncertainty of the abundances derived from the uncertainties in the atmospheric parameters and $E W \mathrm{~s}$.

\begin{tabular}{ccccccc}
\hline \hline Element & $\sigma_{T_{\text {eff }}}$ & $\sigma_{\log g}$ & $\sigma_{\xi}$ & $\sigma_{[\mathrm{Fe} / \mathrm{H}]}$ & $\sigma_{W}$ & $\sigma_{\text {total }}$ \\
\hline $\mathrm{Na}$ & +0.03 & -0.02 & -0.02 & 0.00 & +0.04 & 0.06 \\
$\mathrm{Mg}$ & +0.04 & -0.05 & -0.02 & 0.00 & +0.04 & 0.08 \\
$\mathrm{Al}$ & +0.03 & -0.01 & -0.01 & -0.01 & +0.08 & 0.09 \\
$\mathrm{Si}$ & 0.00 & +0.06 & -0.01 & +0.01 & +0.05 & 0.08 \\
$\mathrm{Ca}$ & +0.04 & -0.03 & -0.03 & -0.01 & +0.05 & 0.08 \\
$\mathrm{Sc}$ & -0.01 & +0.15 & -0.01 & +0.03 & +0.04 & 0.16 \\
$\mathrm{Ti}$ & +0.05 & +0.02 & -0.03 & 0.00 & +0.05 & 0.08 \\
$\mathrm{~V}$ & +0.07 & -0.01 & -0.01 & 0.00 & +0.04 & 0.08 \\
$\mathrm{Cr}$ & +0.04 & +0.03 & -0.02 & +0.07 & +0.06 & 0.11 \\
$\mathrm{Mn}$ & +0.05 & 0.00 & -0.02 & 0.00 & +0.04 & 0.07 \\
$\mathrm{FeI}$ & +0.03 & +0.02 & -0.02 & 0.00 & +0.05 & 0.06 \\
$\mathrm{FeII}$ & -0.04 & +0.17 & -0.03 & +0.03 & +0.06 & 0.19 \\
$\mathrm{Co}$ & +0.03 & +0.04 & 0.00 & +0.01 & +0.04 & 0.06 \\
$\mathrm{Ni}$ & +0.05 & +0.07 & 0.00 & +0.03 & +0.07 & 0.11 \\
$\mathrm{Cu}$ & +0.04 & +0.05 & -0.03 & +0.01 & +0.11 & 0.13 \\
$\mathrm{Zn}$ & -0.01 & +0.11 & -0.03 & +0.03 & +0.07 & 0.14 \\
$\mathrm{Sr}$ & +0.08 & -0.08 & -0.04 & +0.02 & +0.14 & 0.19 \\
$\mathrm{Y}$ & +0.01 & +0.09 & -0.03 & +0.04 & +0.07 & 0.12 \\
$\mathrm{Zr}$ & +0.03 & +0.08 & -0.06 & +0.01 & +0.06 & 0.12 \\
$\mathrm{Ba}$ & +0.01 & +0.01 & -0.01 & +0.05 & +0.03 & 0.06 \\
$\mathrm{La}$ & +0.02 & +0.12 & -0.06 & +0.03 & +0.12 & 0.18 \\
$\mathrm{Ce}$ & +0.01 & +0.09 & -0.04 & +0.03 & +0.07 & 0.12 \\
$\mathrm{Nd}$ & +0.02 & +0.15 & -0.04 & +0.03 & +0.07 & 0.17 \\
$\mathrm{Sm}$ & +0.02 & +0.15 & -0.04 & +0.03 & +0.08 & 0.18 \\
$\mathrm{Eu}$ & 0.00 & +0.15 & -0.02 & +0.03 & +0.05 & 0.16 \\
$\mathrm{Gd}$ & 0.00 & +0.15 & -0.01 & +0.02 & +0.09 & 0.18 \\
\hline & & & & & &
\end{tabular}

\section{HR 1326}

HR 1326 is a supposedly normal giant included in the analysis. It has a solar metallicity and an almost solar abundance pattern, as shown in Fig. 7. There is no chemical peculiarity except for an apparent deficiency of $\mathrm{Na}$ at a level higher than $2 \sigma$.

\section{HR 2392}

HR 2392 (Fig. 7) is a classical barium star with $[\mathrm{Fe} / \mathrm{H}]=$ -0.09 . It was classified as such by Bidelman \& Keenan (1951). According to Warner (1965) it has a Ba3 index. McClure (1983) found this star to have a variable radial velocity and an orbital period of $457.7 \pm 2.7$ days. However, the search for a white dwarf companion resulted in negative detection with IUE (Dominy \& Lambert 1983) and an inconclusive detection with HST (Böhm-Vitense et al. 2000)

The light elements all have solar levels except for $\mathrm{Na}$, which is deficient. In agreement with Zacs (1994) we found this star to be overabundant in heavy elements, as shown in Fig. 7. The s-process elements have a mean overabundance of +1.2 dex. The r-process dominated elements Sm and Eu are also overabundant, whereas Gd shows a normal abundance.

\section{HR 4608}

HR 4608 (Fig. 7) is a mild barium star with [Fe/H] -0.35 dex In the catalogue by $\mathrm{Lu}$ (1991) it has a Ba1 index. McClure (1983) has found no indication for radial velocity variability, but Böhm-Vitense et al. (2000) detected UV-flux excesses that might indicate a white dwarf companion. Udry et al. (1998b) derived a minimum period of $P>4700$ days for this system. The light elements show a solar pattern, except for a larger than $2 \sigma$ deficiency of $\mathrm{Na}$. The s-process elements show a mean overabundance of +0.6 dex. No r-process overabundance can be established with statistical significance.

\section{HR 5058}

HR 5058 (Fig. 8) is another classical barium star, first identified by Bidelman \& Keenan (1951). Its metallicity is $[\mathrm{Fe} / \mathrm{H}]=-0.12$, and Warner (1965) classified this star as Ba3. HR 5058 is a member of a binary system with a white dwarf companion identified by Böhm-Vitense et al. (2000).

This star was previously analyzed by Luck \& Bond (1991). Our results are in good agreement with theirs. The s-process elements have a mean overabundance of +1.0 dex. The abundance pattern shows a surprising overabundance of $\mathrm{Cu}$. We can not compare the abundance of $\mathrm{Cu}$ with previous results since ours seems to be the first $\mathrm{Cu}$ abundance determination for this star, therefore we caution that this intriguing result should await further confirmation. The r-process elements $\mathrm{Sm}$ and $\mathrm{Gd}$ show a statistically significant overabundance, $\sim 0.5 \mathrm{dex}$, whereas Gd seems to be normal, similarly to what was found for HR 2392.

\section{HR 5802}

HR 5802 is another mild barium star classified by Warner (1965) as Ba1.0. It has a solar metallicity. McClure (1983) argues this star to have a variable radial velocity. Its abundance pattern is shown in Fig. 8 where we note the light elements to follow a solar pattern except for a larger than $2 \sigma$ deficiency of $\mathrm{Na}$. In this star the lighter s-process elements $(\mathrm{Sr}, \mathrm{Y}$ and $\mathrm{Zr}$ ) have a marked overabundance, with a mean of +0.56 dex, while the heavier ones (Ba, $\mathrm{La}, \mathrm{Ce}$ and $\mathrm{Nd}$ ) seem to be only slightly overabundant although statistically they are normal.

Zacs (1994) analyzed this star and found results that agree with ours within the uncertainties. There are only two exceptions, $\mathrm{Zr}$ for which he found solar abundance and Ba for which he found a larger excess.

\section{HR 7321}

HR 7321 is another example of a mild barium star with metallicity slightly lower than solar, $[\mathrm{Fe} / \mathrm{H}]=-0.19$ dex. Its abundance pattern is shown in Fig. 8. In the catalogue of $\mathrm{Lu}$ (1991) it has a $\mathrm{Ba} 0.5$ index. Most elements follow a solar pattern except for the larger than $2 \sigma$ deficiencies of $\mathrm{Na}$ and $\mathrm{Mn}$. There is a good agreement between our abundances and those by Zacs (1994), except for $\mathrm{Zr}$ which he founds to be underabundant. As for HR 5802 the heavier s-process elements are statistically normal while the lighter ones have only a slightly larger than $2 \sigma$ enhancement with a mean value of +0.35 dex.

\section{HR 8115}

HR 8115 (Fig. 8) is another example of a mild barium star and was classified as Bal by $\mathrm{Lu}$ (1991). Griffin \& Keenan (1992) found the star to be a binary with a rather long period, 18 years. Dominy \& Lambert (1983) found a UV flux excess that probably indicates a white dwarf companion.

Its metallicity is solar. Up to the iron-peak, the only deviations from a solar abundance pattern are deficiencies of $\mathrm{V}$ and $\mathrm{Mn}$ at the $2 \sigma$ level. As in HR 5802 and HR 7321, the heavier s-process elements seem to be slightly enhanced but with low statistical significance. The lighter s-process elements, $\mathrm{Sr}$, $\mathrm{Y}$ and $\mathrm{Zr}$ seem to be more clearly enhanced, particularly $\mathrm{Sr}$. 
Table 6. Visual magnitude, $V_{T}$, parallax, absolute magnitude $M_{V T}$, bolometric correction BC, bolometric magnitudes and luminosities of the sample stars. The last column refers to the luminosity uncertainty due to error propagation of the involved quantities.

\begin{tabular}{ccccccccc}
\hline \hline Star & $V_{T}$ & $\pi(\mathrm{mas})$ & $M_{V T}$ & $\mathrm{BC}$ & $M_{\mathrm{BOL}}$ & $\sigma_{M_{\mathrm{BOL}}}$ & $\log \left(L_{\star} / L_{\odot}\right)$ & $\sigma_{\log \left(L_{\star} / L_{\odot}\right)}$ \\
\hline HR 440 & 4.05 & 22.15 & +0.78 & -0.34 & +0.44 & 0.04 & 1.75 & 0.02 \\
HR 649 & 4.47 & 9.01 & -0.76 & -0.22 & -0.98 & 0.21 & 2.32 & 0.09 \\
HR 1016 & 5.60 & 7.75 & +0.04 & -0.23 & -0.19 & 0.14 & 2.00 & 0.05 \\
HR 1326 & 3.98 & 27.85 & +1.21 & -0.39 & -0.81 & 0.03 & 1.60 & 0.01 \\
HR 2392 & 6.39 & 8.25 & +0.97 & -0.28 & +0.68 & 0.16 & 1.65 & 0.07 \\
HR 4608 & 4.22 & 19.08 & +0.63 & -0.29 & +0.34 & 0.06 & 1.79 & 0.03 \\
$\epsilon$ Vir & 2.95 & 31.90 & +0.47 & -0.23 & +0.24 & 0.04 & 1.83 & 0.02 \\
HR 5058 & 5.24 & 15.73 & +1.23 & -0.34 & +0.89 & 0.08 & 1.57 & 0.03 \\
HR 5802 & 5.36 & 13.89 & +1.07 & -0.26 & +0.82 & 0.09 & 1.60 & 0.04 \\
HR 7321 & 6.52 & 6.68 & +0.64 & -0.33 & +0.31 & 0.21 & 1.80 & 0.08 \\
HR 8115 & 3.31 & 21.62 & -0.01 & -0.29 & -0.31 & 0.03 & 2.05 & 0.01 \\
HR 8204 & 3.86 & 8.19 & -1.57 & -0.18 & -1.76 & 0.18 & 2.63 & 0.07 \\
HD 205011 & 6.54 & 6.31 & +0.54 & -0.34 & +0.20 & 0.15 & 1.85 & 0.06 \\
HR 8878 & 5.21 & 9.56 & +0.11 & -0.52 & -0.41 & 0.13 & 2.09 & 0.05 \\
\hline
\end{tabular}

Abundances from Ba to Ce could be normal, and from $\mathrm{Nd}$ to $\mathrm{Gd}$ are solar. Our abundances are in relatively good agreement with the abundances derived by Pilachowski (1977) and Yushchenko et al. (2004). We tentatively suggest this star to be a mild barium star.

\section{HR 8204}

HR 8204 (Fig. 8) is a classical barium star with $[\mathrm{Fe} / \mathrm{H}]=$ -0.09 dex. It was first recognized by Bidelman \& Keenan (1951) and was the first barium system where a UV flux excess was identified (Böhm-Vitense 1980). It is classified by Warner (1965) as $\mathrm{Ba} 2$.

The light elements show a solar pattern, except by a larger than $2 \sigma$ underabundance of $\mathrm{Na}$ and an overabundance of $\mathrm{Al}$. $\mathrm{Sc}$ is probably deficient, and all the s-process elements have overabundances larger than +1.0 dex, with $\mathrm{Sr}$ reaching $+2.2 \mathrm{dex}$; the mean overabundance is +1.4 dex. This star was previously analyzed by Zacs (1994) and our results are in agreement with his within the uncertainties. The r-process elements $\mathrm{Sm}$ and $\mathrm{Eu}$ are not overabundant.

\section{HR 8878}

HR 8878 (Fig. 8) is the most metal deficient star in our analysis, $[\mathrm{Fe} / \mathrm{H}]=-0.67$. Its abundance pattern is enriched in $\mathrm{Ti}$, $\mathrm{Mg}$ and $\mathrm{Al}$, while $\mathrm{Na}$ is deficient. $\mathrm{Mn}$ is also deficient at the $2 \sigma$ level. These values are expected for normal metal deficient stars, except for $\mathrm{Na}$, which should track Al (Edvardsson et al. 1993; McWilliam 1997). We found no s-process element to be overabundant, in contrast with Zacs (1994), who found Ba to be enhanced, we found a deficiency of this element at the $2 \sigma$ level, as expected for mildly metal poor stars. Our abundances classify this object as a normal metal poor giant star.

\section{HD 205011}

HD 205011 (Fig. 9) is another mild barium star analyzed by Zacs (1994) and classified by Lu (1991) as Ba2. Its metallicity is slightly lower than solar, $[\mathrm{Fe} / \mathrm{H}]=-0.14$ dex. McClure (1983) found the star to have a variable radial velocity. Our abundances are generally in good agreement with those by Zacs (1994) except for $\mathrm{Zr}$ which he found to be solar. Na, V and Mn seem to have a larger than $2 \sigma$ deficiency. The s-process elements have a mean overabundance of $+0.66 \mathrm{dex}$, and this star seems to be intermediate between mild and classical barium stars. The r-process elements have normal abundances.

\subsection{Masses and ages}

We also estimated the masses and ages of the sample stars by placing them in the HR diagram with isochrones and theoretical evolutionary tracks from the Geneva group (Charbonnel et al. 1993; Schaerer et al. 1993; and Schaller et al. 1992). We made use of the previously derived $T_{\text {eff }}$ and calculated the luminosities as follows.

With parallaxes and visual magnitudes, $V_{T}$, from the Hipparcos catalogue (ESA 1997), and bolometric corrections from Landolt-Börnstein (1982), we calculated the absolute bolometric magnitudes. Thus, adopting the solar bolometric magnitude in the Tycho system, $M_{\mathrm{Bol}_{\odot}}=4.81$, we calculated the luminosities using $\log \left(L_{\star} / L_{\odot}\right)=-0.4\left(M_{\mathrm{Bol}_{\star}}-M_{\mathrm{Bol}_{\odot}}\right)$. The magnitudes and luminosities with respective uncertainties are listed in Table 6.

For all stars but HR 8878, the masses and ages were linearly interpolated from the values obtained by placing the stars in theoretical diagrams for two metallicities, $[\mathrm{Fe} / \mathrm{H}]=-0.40$ and $[\mathrm{Fe} / \mathrm{H}]=+0.00$. The mass and age of HR 8878 were obtained by using a third diagram with $[\mathrm{Fe} / \mathrm{H}]=-0.72$. The masses and ages thus calculated are listed in Table 7 . Figure 10 shows the region of the HR diagram where the sample stars are located.

We also estimated the "astrometric" $\log g s$ using the derived masses of the stars, as done for $\epsilon$ Vir in Sect. 3.1. The $\log g s$ and its uncertainty, calculated in a straightforward way by the error propagation of the involved quantities, are also listed in Table 7. The agreement between the ionization and astrometric values of $\log g$ is good within the uncertainties, except for HR 8204, which has a significantly lower spectroscopic $\log g$. The barium and mild barium giants of our sample define together a narrow range in stellar mass, 1.9 to $4.2 M_{\odot}$, and age, 0.2 to $1.0 \mathrm{Gyr}$, indicating that they most probably share similar evolutionary stages.

\section{Discussion}

\subsection{The mild barium stars}

With the first few orbital parameters for barium stars, McClure et al. (1980) were able to confirm the binarity of most of the strong barium stars $(\mathrm{Ba}>2)$ they analyzed. Based on that it 
Table 7. Masses, ages and an evolutionary estimates of the surface gravity of the sample stars.

\begin{tabular}{ccccc}
\hline \hline Star & Mass $\left(M_{\odot}\right)$ & Age $(\mathrm{Gy})$ & $\log g$ & $\sigma_{\log g}$ \\
\hline HR 440 & 1.9 & 0.8 & 2.64 & 0.03 \\
HR 649 & 3.6 & 0.1 & 2.47 & 0.10 \\
HR 1016 & 3.0 & 0.2 & 2.70 & 0.07 \\
HR 1326 & 1.5 & 1.3 & 2.65 & 0.03 \\
HR 2392 & 2.3 & 0.5 & 2.87 & 0.08 \\
HR 4608 & 2.3 & 0.5 & 2.72 & 0.04 \\
$\epsilon$ Vir & 2.9 & 0.3 & 2.85 & 0.03 \\
HR 5058 & 1.9 & 0.9 & 2.82 & 0.05 \\
HR 5802 & 2.4 & 0.5 & 2.97 & 0.05 \\
HR 7321 & 2.2 & 0.5 & 2.66 & 0.10 \\
HR 8115 & 3.0 & 0.2 & 2.58 & 0.03 \\
HR 8204 & 4.2 & 1.0 & 2.27 & 0.09 \\
HD 205011 & 2.2 & 0.5 & 2.61 & 0.08 \\
HR 8878 & 1.0 & 6.2 & 1.87 & 0.07 \\
\hline
\end{tabular}

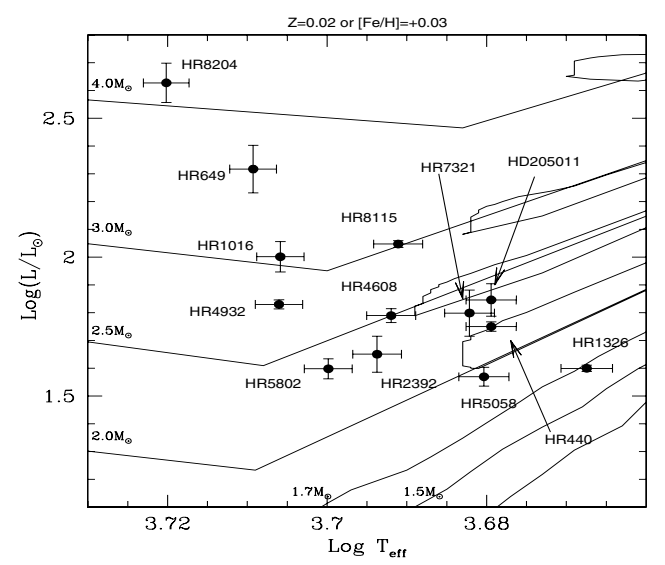

Fig. 10. The HR diagram with our stars and theoretical tracks from Schaller et al. (1992) for $[\mathrm{Fe} / \mathrm{H}]=+0.03$. The most metal deficient star of our sample, HR 8878, is not shown in this plot. The normal stars are shown as open squares, the mild barium stars as circles and the barium stars as triangles.

was possible to conclude that the strong barium stars were all probable members of binary systems.

The same conclusion, however, was not possible in the case of the mild barium stars. Most of the stars for which no radial velocity variability was found belonged to the mild barium class. A firm conclusion was not possible although they mostly seemed to be members of a binary system (McClure 1984).

It was then suggested that mild barium stars could have wider separations between the components (Böhm-Vitense et al. 1984), which would require observations spanning longer durations to detect their binary nature. Some mild barium stars were, however, found to be misclassified, as in Smith \& Lambert (1987). Naturally this leads to the question of how many misclassified objects there are in the mild barium star lists.

More recently, based on an extended sample of orbital elements (Udry et al. 1998a,b), Jorissen et al. (1998) were able to confirm the binary status for 34 among 40 mild barium stars. Thus it was shown that the frequency of detected binaries is compatible with all mild barium stars belonging to binary systems. However the frequency of detected binarity in strong barium stars is still larger, 35 out of 37.

Jorissen et al. (1998) have also shown that mild barium stars are not restricted to long period systems. This argues against the suggestion that the non-detection of binarity in some mild barium systems is due solely to wider separations. In this work we found the stars HR 649 and HR 1016 not to be mild barium stars, in contrast to what was generally accepted in the literature. Moreover, since HR 649 seems to have a white dwarf companion (Böhm-Vitense \& Johnson 1985) the very fact it exists shows that binarity is not a sufficient condition to form a barium system.

Particularly we note that many works on general characteristics of barium (and mild barium stars) such as kinematics (Gómez et al. 1997) or distribution along the HR diagram (Bergeat \& Knapik 1997) adopted lists of stars that have never been targets of an abundance analysis based on high resolution spectra. Thus, many of the stars have only a tentative classification as mild barium. If a significant number of tentative mild barium stars are in fact misclassified, these studies may be suffering from important biases.

The results by Jorissen et al. (1998) indicate that a wider separation is not the parameter controlling the difference in the abundances between mild and strong barium stars. They argue that metallicity may be the main parameter. Thus, the increasing level of overabundances seen in mild barium, strong barium and population II CH-stars would correspond to a sequence of older, and thus more metal-poor, populations. A discussion of this suggestion is presented below but we can state in advance that barium and mild barium stars do not seem to have different metallicities.

Thus, there are still important open questions on the nature of the mild barium systems. Moreover, there seems to be no work in the literature on confirming the abundance peculiarities of a large sample of mild barium stars. We are currently analyzing a large sample of southern, tentative mild barium systems in order to derive their barium abundances, their metallicities and atmospheric parameters. These results and a discussion on this subject will be presented in a forthcoming paper.

\subsection{What differentiates barium and mild barium stars?}

The s-process nucleosynthesis has long being recognized to be a complex process. In order to explain the observations it was divided into three components, the main, strong and weak components. The weak component is thought to occur during He burning in massive stars and to be responsible for isotopes from Fe to Sr. The main and strong components are thought to occur in AGB stars (Busso et al. 1999). The strong component is needed to build ${ }^{208} \mathrm{~Pb}$, which has been shown to happen in metalpoor AGBs (Travaglio et al. 2001). The main component, in low mass AGBs, is necessary to build s-elements from $\mathrm{Sr}$ to $\mathrm{Pb}$.

In low mass AGBs the neutrons flux seems to be mostly due to the ${ }^{13} \mathrm{C}(\alpha, n){ }^{16} \mathrm{O}$ reaction in a radiative layer during the interval between thermal pulses. A marginal activation of the ${ }^{22} \mathrm{Ne}(\alpha, n){ }^{25} \mathrm{Mg}$ reaction in convective conditions during the thermal pulses may also occur (Busso et al. 2001).

Barium (and mild barium) stars are thought to be the result of mass transfer from s-process enriched AGB stars. Thus their overabundances should follow the general pattern expected for AGBs, as was shown elsewhere (Busso et al. 1995, 2001). Following these results, we will here compare and discuss the observed patterns in barium and mild barium stars, in order to highlight some differences and similarities between these groups of stars.

Following Luck \& Bond (1991), the [hs/ls] ratio has been widely used as an indicator of s-process efficiency. [hs] stands for the mean abundance of the "heavy" s-process elements of the Ba peak, and [ls] is the same for the "light" s-process elements 


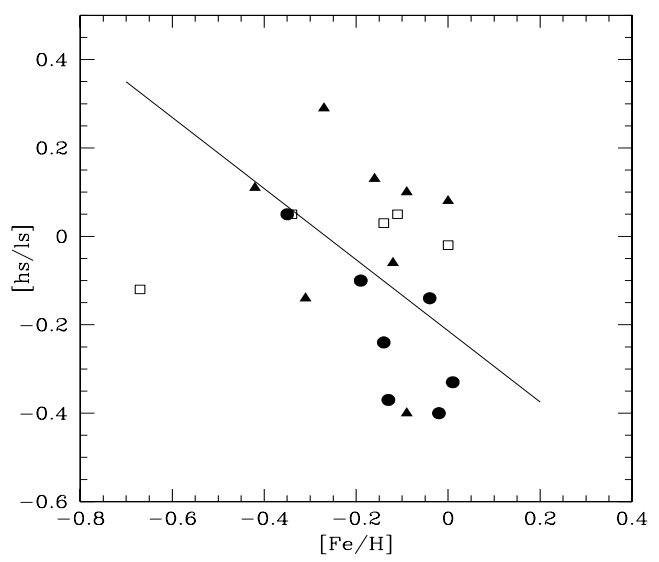

Fig. 11. The ratio $[\mathrm{hs} / \mathrm{ls}]$ vs. $[\mathrm{Fe} / \mathrm{H}]$ for our sample stars. hs/Fe] is the mean abundance of $\mathrm{Ba}, \mathrm{La}, \mathrm{Ce}$ and $\mathrm{Nd},[\mathrm{ls} / \mathrm{Fe}]$ is the mean of $\mathrm{Sr}, \mathrm{Zr}$ and Y. In this plot the normal stars are represented as open squares, the mild barium stars as circles and the barium stars as triangles. The solid line is a linear fit to the peculiar giants only, barium and mild barium, excluding the normal giants.

of the $\mathrm{Zr}$ peak. These nuclei have neutron magic numbers and thus have a low cross section against further neutron captures.

Various authors adopted different elements to calculate [hs] and [ls]. Here we use $\mathrm{Sr}, \mathrm{Zr}$ and $\mathrm{Y}$ to calculate [ls] and $\mathrm{Ba}, \mathrm{La}$, $\mathrm{Ce}$ and $\mathrm{Nd}$ to calculate [hs]. We calculated this ratio for our stars and also for stars analyzed in the literature with high resolution, high signal to noise spectra (Boyarchuk et al. 2002; Liang et al. 2003; Antipova et al. 2004) whenever abundances for the same elements were available.

This ratio has been shown to be a (complex) function of the neutron exposure (Busso et al. 2001). Figure 11 shows the plot of $[\mathrm{hs} / \mathrm{ls}] \mathrm{vs}$. $[\mathrm{Fe} / \mathrm{H}]$. In this figure the normal giants are shown as open squares, the mild barium stars as circles and the barium stars as triangles. The general trend observed in several works is also clear here, there is an anticorrelation between [hs/ls] and $[\mathrm{Fe} / \mathrm{H}]$. In the restricted metallicity range we are considering here a direct correlation between [hs/ls] and the neutron exposure is not possible (Busso et al. 2001), since the several theoretical tracks overlap. It is however interesting to note that there is a general trend for the mild barium stars to fall below the barium stars of same metallicity.

Although the scatter seems to be large, the standard deviation from a linear fit (using only barium and mild barium stars) is $\sigma=0.20$, a value that can be fully ascribed to observational uncertainties. The few normal giants plotted show little scatter $(\approx 0.08 \mathrm{dex})$ around $[\mathrm{hs} / \mathrm{ls}]=0.0 \mathrm{dex}$, as would be expected for a solar scaled mixture.

An important fact that appears in Fig. 11 and is clearer in Fig. 12 is that there seems to be no significant difference in iron abundance between mild barium and barium stars. The two groups have also the same metallicity range of the normal disk giants considered here. The only separation occurs in barium abundance as anticipated by the labels, normal, mild barium and barium giants. Thus, at least with respect of metal content, barium and mild barium stars seem to be members of the same stellar population.

At lower metallicities higher overabundances of s-process elements can be achieved (Busso et al. 2001). This happens because for a given neutron flux the neutron exposure increases with decreasing number of iron group seed nuclei. In addition, in a lower metallicity environment the abundance of the neutron poisons, nuclei that do not take part in the s-process branches but

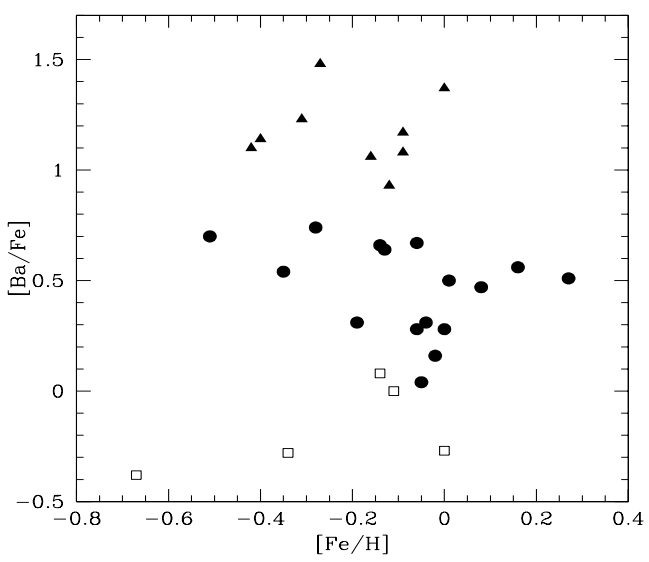

Fig. 12. The barium abundance, $[\mathrm{Ba} / \mathrm{Fe}]$, vs. iron abundance, $[\mathrm{Fe} / \mathrm{H}]$. The symbols have the same meaning as in Fig. 19. It is clear that there is no separation between barium and mild barium stars with respect to the iron abundance.

also capture neutrons, is also smaller. Thus it has been argued that barium stars could be members of a more metal-poor population than mild barium stars. The data presented here (Figs. 11 and 12) do not support that suggestion.

Having discarded the hypothesis of a more metal rich origin for mild barium stars and that they are not restricted to longperiod systems (Jorissen et al. 1998), other scenarios for their formation should be found.

The essence behind the proposition of different metallicities to explain the difference in overabundances lies in the different neutron exposure that the materials would have been subjected to. Even though we discarded this difference in the metallicities, there could be another mechanism affecting the neutron exposure, so that the material in mild barium stars could still be the result of a smaller neutron exposure.

For a given metallicity, a lower neutron exposure should favor the production of nuclei in the $\mathrm{Zr}$ peak. Increasing the neutron exposures should favor the formation of the Ba peak nuclei. This means that, for a given metallicity, the ratio of the Ba peak to the $\mathrm{Zr}$ peak abundances ([Ba/Zr] or [hs/ls]) should increase with increasing neutron exposure and hence with increasing Ba peak abundances. One has to note, however, that for a given neutron exposure a decreasing metallicity would produce the same effect. Thus, we search for any significant sign of this possible effect in Figs. 13 and 14. These figures show plots of $[\mathrm{Ba} / \mathrm{Zr}]$ vs. $[\mathrm{Ba} / \mathrm{Fe}]$ and $[\mathrm{hs} / \mathrm{ls}]$ vs. $[\mathrm{hs} / \mathrm{Fe}]$, respectively, for the whole sample, comprising all the metallicity range for which information was available.

A large scatter can be seen in both plots (Figs. 13 and 14) in the sense that a given $[\mathrm{Ba} / \mathrm{Zr}]$ ratio (or $[\mathrm{hs} / \mathrm{ls}]$ ) seems to correspond to a variety of $[\mathrm{Ba} / \mathrm{Fe}]$ (or $[\mathrm{hs} / \mathrm{Fe}]$ ) values. In Fig. 13 there are two stars, HD 26886, a mild barium star, and HD 50082, a barium star, that have a higher $[\mathrm{Ba} / \mathrm{Zr}]$ than the bulk of the other stars. Both were analyzed by Liang et al. (2003). Their larger $[\mathrm{Ba} / \mathrm{Zr}]$ are due to a smaller $\mathrm{Zr}$ abundance when compared to stars with similar Ba abundance. Without these two stars the average $[\mathrm{Ba} / \mathrm{Zr}]$ for the mild barium stars is $+0.00 \pm 0.20$ and $+0.04 \pm 0.12$ for the barium stars, or $+0.06 \pm 0.27$ and $+0.11 \pm 0.20$ including them, respectively. In any case, as is clear from the plot, there is no significant difference in the $[\mathrm{Ba} / \mathrm{Zr}]$ ratio between barium and mild barium stars. We have also plotted equivalent diagrams, $[\mathrm{Ba} / \mathrm{Y}]$ vs. $[\mathrm{Ba} / \mathrm{Fe}],[\mathrm{La} / \mathrm{Zr}]$ vs. $[\mathrm{La} / \mathrm{Fe}]$, and $[\mathrm{La} / \mathrm{Y}]$ vs. $[\mathrm{La} / \mathrm{Fe}]$, and, similarly, no clear trend of an increasing ratio of a heavy to a light s-process element with increasing 


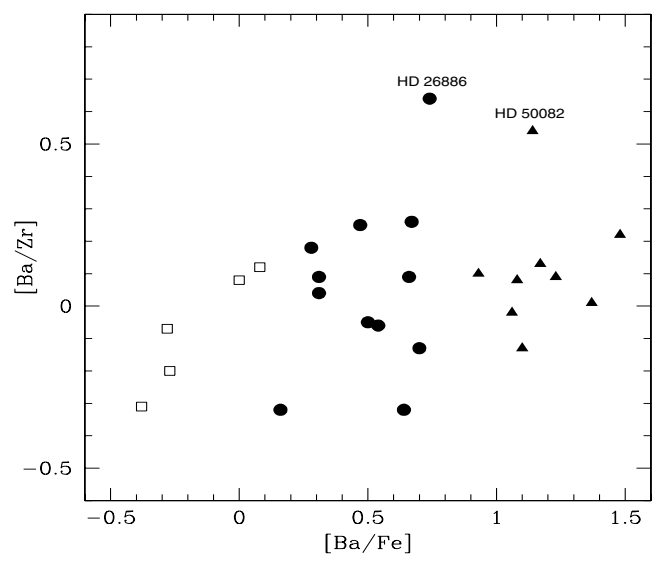

Fig. 13. Plot of $[\mathrm{Ba} / \mathrm{Zr}]$ vs. $[\mathrm{Ba} / \mathrm{Fe}]$ for the whole sample being considered. The symbols are the same as in the previous plots.

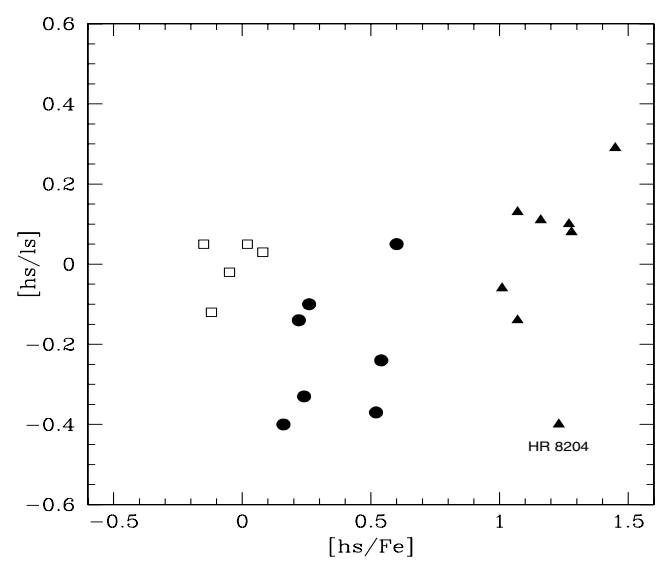

Fig. 14. Plot of [hs/ls] vs. [hs/Fe] for the whole sample being considered. The symbols are the same as in the previous plots.

excess of the corresponding heavy s-process element could be discerned with any statistical significance.

The number of points in Fig. 14 is smaller, since for some stars abundances for all the elements defining [hs] and [ls] were not available. Nevertheless, in this plot there seems to be a trend of increasing $[\mathrm{hs} / \mathrm{ls}]$ with increasing $[\mathrm{hs} / \mathrm{Fe}]$, when considering only the peculiar giants. The barium star with smaller $[\mathrm{hs} / \mathrm{ls}]$ in the plot is HR 8204 . Its low [hs/ls] is strongly influenced by the high $\mathrm{Sr}$ overabundance, based on only one line, which increases its [ls] mean. The average [hs/ls] for the mild barium stars is $-0.22 \pm 0.16$ and for the barium stars is $+0.01 \pm 0.21$, or $+0.07 \pm 0.14$ without HR 8204. A possible conclusion might be that, when one considers all the available $[\mathrm{X} / \mathrm{Fe}]$ ratios in composing the [hs] and [ls] means, and in spite of large scatter, mild barium stars seem to have a slightly lower level of neutron exposure than classical barium stars, even though their metallicity range is exactly the same.

However, we still have to be very careful in drawing a conclusion. The position of HR 8204 seems to indicate that it is possible to produce a barium star with a [hs/ls] as low as that observed in some mild barium giants. Moreover, the mild barium star with the highest $[\mathrm{Ba} / \mathrm{Zr}]$ in Fig. 13 is not included in Fig. 14, due to the lack of the abundances of the necessary elements, and therefore we cannot be sure there are not mild barium stars with [hs/ls] ratios as high as the peak observed for the barium giants. The addition of more points like those could blur the weak correlation seen in Fig. 14. Thus, although we have indications that the material in barium stars was subject to a higher neutron exposure, a firm conclusion should await an increase in the sample.

In the case mild barium and barium stars do share the same range in neutron exposure their differences should be related to yet another factor other than either neutron exposure, metallicity or larger orbital period. The difference could, for example, be connected to the mass range of the progenitors, and the associated nucleosynthetic processes, and their ability to mix s-process enriched material to the surface during the third dredge-up. It could also be related to the convective mixing of the barium stars themselves during the RGB, the first dredge-up, acting to dilute the overabundances.

We do not have ${ }^{12} \mathrm{C} /{ }^{13} \mathrm{C}$ or $\mathrm{C}$ and $\mathrm{N}$ abundances for the sample stars, hence we cannot discuss their evolutionary status in detail. However, in Fig. 10 we see that both barium and mild barium stars of our sample seem to share the same region in the HR diagram and to have similar masses. A great difference in the convective mixing efficiency in this restricted range of stellar masses is not expected (Schaller et al. 1992). We cannot discard the possibility that the origin of the differences lie in a complex combination of all these phenomena, mixing, mass loss, and neutron exposure with some role played by metallicity dependence or orbital separation.

A straightforward conclusion on the origin of the different overabundances in barium and mild barium is not yet possible. We discarded the hypothesis of different metallicities, at least in the range of parameters defined by our sample, but we also showed that there seems to be a difference in the neutron exposure range. Further observational work on extending the sample of barium and mild barium stars with detailed abundance analysis is necessary as well as additional theoretical work on formation scenarios for these systems.

\subsection{Copper in s-process enhanced stars}

The nucleosynthetic sites of $\mathrm{Cu}$ production are still poorly known. Sneden et al. (1991) derived $\mathrm{Cu}$ abundances for a large sample of field and globular cluster stars and suggested that $\mathrm{Cu}$ was mainly produced by the weak component of the s-process in massive stars, with only a small contribution of type I supernovae and of the main component of the s-process in intermediate mass stars. Bisterzo et al. (2004) discuss a collection of $\mathrm{Cu}$ abundances from the literature of a variety of systems, field stars (from halo, thick and thin disk), bulge-like stars, globular cluster stars and stars from dwarf spheroidal galaxies. They conclude that the $\mathrm{Cu}$ behavior can be explained as the result of an efficient weak s-process in massive stars. McWiliam \& SmeckerHane (2005) show that this conclusion is consistent with the $\mathrm{Cu}$ abundances in the Sagittarius dwarf spheroidal and the chemical evolution scenario earlier proposed to explain the Mn abundances of this system (McWilliam et al. 2003).

On the other hand, Matteucci et al. (1993) argue that a long lived process, such as type Ia supernovae, is necessary to bring the observations and theory of the Galactic chemical evolution of $\mathrm{Cu}$ into agreement. Mishenina et al. (2002), by means of an abundance analysis of a large sample of metalpoor halo and thick disc stars, also conclude that the non-linear trend of $[\mathrm{Cu} / \mathrm{Fe}]$ with $[\mathrm{Fe} / \mathrm{H}]$ is best explained if the bulk of $\mathrm{Cu}$ comes from explosive nucleosynthesis in type Ia supernovae. Abundances of $\mathrm{Cu}$ in $\omega$ Cen (Cunha et al. 2002) and other globular clusters (Simmerer et al. 2003) also seem to indicate that $\mathrm{Cu}$ is mainly produced by type Ia supernovae.

Further investigation, both on theoretical yields and on the observational behavior of $\mathrm{Cu}$ in a variety of systems, is still 


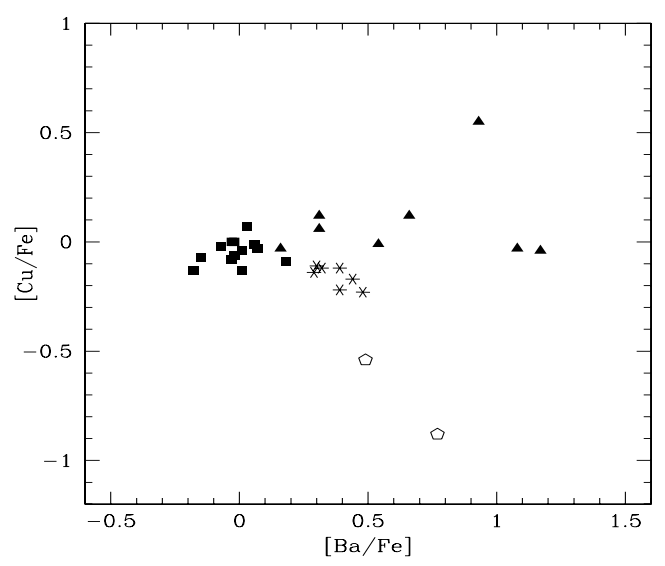

Fig. 15. Plot of $[\mathrm{Cu} / \mathrm{Fe}]$ vs. $[\mathrm{Ba} / \mathrm{Fe}]$. In this figure the triangles are the barium and mild barium stars analyzed in this work. The squares are normal disc stars from Castro et al. (1999). The asterisks are s-process enriched dwarfs of the UMaG also from Castro et al. (1999). The open symbols are yellow symbiotic stars from Pereira \& Porto de Mello (1997) and Pereira et al. (1998).

needed in order to understand the nucleosynthetic origin of $\mathrm{Cu}$. In addition to this discussion, Castro et al. (1999) derived $\mathrm{Cu}$ and $\mathrm{Ba}$ abundances for a sample of barium enhanced dwarfs from the UMaG (Soderblom \& Mayor 1993) and found the existence of an anticorrelation between $[\mathrm{Cu} / \mathrm{Fe}]$ and $[\mathrm{Ba} / \mathrm{Fe}]$. This anticorrelation is reinforced by the $\mathrm{Cu}$ deficiency observed in two yellow symbiotic stars that are s-process enhanced (Pereira \& Porto de Mello 1997; Pereira et al. 1998). This is a possible indication that besides being built by the s-process, $\mathrm{Cu}$ could also be a seed to the production of heavier elements. Its depletion in s-process enriched stars could be a sign of preferential use as seed. One of the goals of this work was to verify whether this anticorrelation is also present in barium stars.

Figure 15 shows the plot of $[\mathrm{Cu} / \mathrm{Fe}]$ vs. $[\mathrm{Ba} / \mathrm{Fe}]$ for the UMaG stars from Castro et al. (1999), the barium and mild barium stars of this work, the two yellow symbiotic stars from Pereira \& Porto de Mello (1997) and Pereira et al. (1998) and a sample of normal disk stars also from Castro et al. (1999). Barium and mild barium stars, however, do not follow the anticorrelation. On the contrary, they seem to follow the plateau defined by the normal disk stars. This result argues that the observed depletion is not a common fact that extends to all s-process enhanced stars. We note however that the origin of this anticorrelation is still not clear and deserves further investigation.

The analysis of HR 6094, a s-process enhanced UMaG member (Porto de Mello \& da Silva 1997) also suggests a depletion of $\mathrm{Mn}$ and excess of $\mathrm{V}$ and Sc. Neither of these effects is seen in the stars we analyzed or in the data of the barium and mild barium stars we collected from the literature. We show in Figs. 16-18 the plots of $[\mathrm{Mn} / \mathrm{Fe}],[\mathrm{V} / \mathrm{Fe}]$ and $[\mathrm{Sc} / \mathrm{Fe}]$ vs. $[\mathrm{Ba} / \mathrm{Fe}]$ respectively. In these the triangles are barium stars, the circles are mild barium stars and the squares normal giants. No clear-cut difference in $\mathrm{Mn}, \mathrm{V}$ or Sc content is apparent between the three group of stars, except for a larger scatter of $\mathrm{Mn}$ and $\mathrm{V}$ in the chemically peculiar stars.

\section{Conclusions}

We carried out a detailed analysis of a sample of eleven barium and mild barium stars and three normal giants using high resolution, high signal to noise spectra. We determined atmospheric

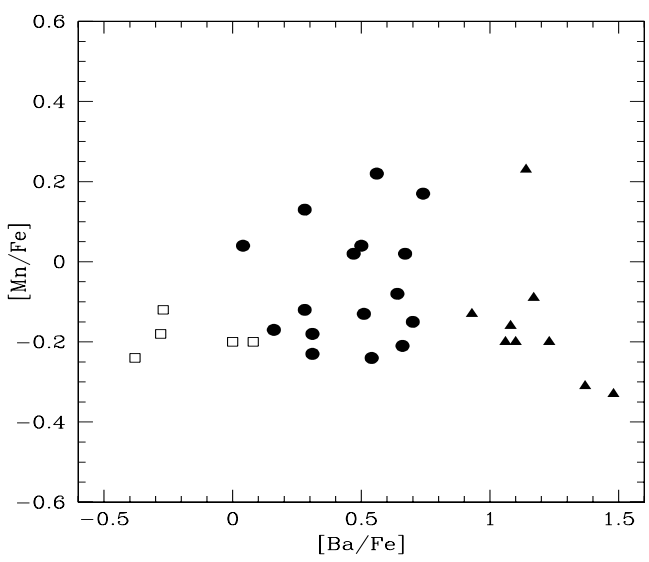

Fig. 16. Plot of $[\mathrm{Mn} / \mathrm{Fe}]$ vs. $[\mathrm{Ba} / \mathrm{Fe}]$. In this figure the triangles are barium stars, the circles are mild barium stars and the open squares are normal giants.

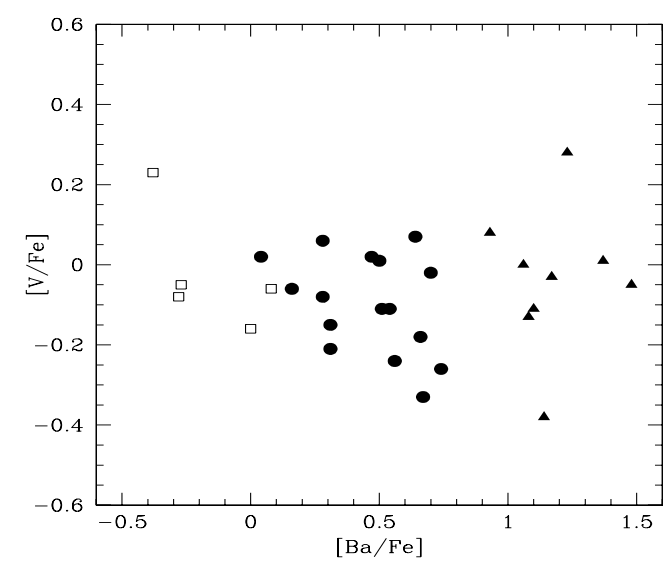

Fig. 17. Plot of $[\mathrm{V} / \mathrm{Fe}]$ vs. $[\mathrm{Ba} / \mathrm{Fe}]$. Symbols are as in Fig. 24.

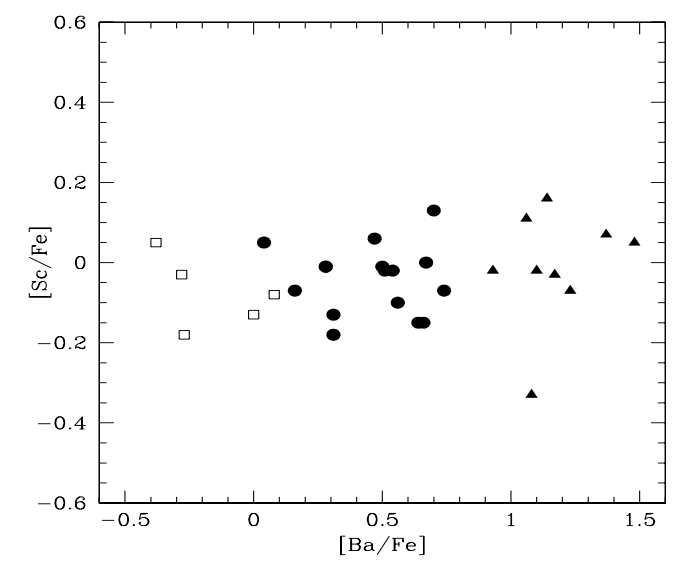

Fig. 18. Plot of [Sc/Fe] vs. [Ba/Fe]. Symbols are as in Fig. 24.

parameters, ages, masses and abundances for twenty-five elements; $\mathrm{Na}, \mathrm{Mg}, \mathrm{Al}, \mathrm{Si}, \mathrm{Ca}, \mathrm{Sc}, \mathrm{Ti}, \mathrm{V}, \mathrm{Cr}, \mathrm{Mn}, \mathrm{Fe}, \mathrm{Co}, \mathrm{Ni}, \mathrm{Cu}$, $\mathrm{Zn}, \mathrm{Sr}, \mathrm{Y}, \mathrm{Zr}, \mathrm{Ba}, \mathrm{La}, \mathrm{Ce}, \mathrm{Nd}, \mathrm{Sm}, \mathrm{Eu}$ and Gd.

We found the stars HR 649 and HR 1016 to be normal red giants, not mild barium stars, in contrast to what was generally accepted in the literature. Since there are other cases in the literature of mild barium stars found to be misclassified (Smith \& Lambert 1987) we suggest that a verification of the peculiar status of a large sample of tentative mild barium stars is very much needed. With this aim we are currently analyzing a large 
sample of mild barium stars, and the results will be presented in a forthcoming paper.

The abundances of barium and mild barium stars were compared and we found that there seems to be no difference in iron abundance between them. The two groups seem have the same metallicity range of normal disk giants and thus barium and mild barium stars seem to be members of the same stellar population.

We found some indications that the material transferred onto barium stars could have been subjected to a higher neutron exposure than that accreted by the mild barium stars. More work, however, is needed to confirm this result. The reasons for this difference are not yet clear. Metallicity does not seem to be an issue, but possibly higher neutron exposures are associated with higher excesses of the heavier s-process elements. Parameters which might be involved are the mass range of the former primaries of these systems, or differences in the mixing processes of the barium stars themselves.

A possible anticorrelation between $[\mathrm{Cu} / \mathrm{Fe}]$ and $[\mathrm{Ba} / \mathrm{Fe}]$ seen in some s-process enriched stars (Castro et al. 1999) was not identified in the barium or mild barium stars of our sample. These seem to follow the plateau defined by the normal disk stars. This result argues that the observed depletion of $\mathrm{Cu}$ in some barium enhanced stars is not a common feature that extends to all s-process enhanced stars. The origin of the anticorrelation deserves further investigation. Possible similar effects for Sc, V and Mn were not identified either in the barium and mild barium stars of our sample.

Acknowledgements. This paper is based on the senior thesis of R.S. G.F.P.M. would like to thank Verne V. Smith, Roberto Gallino and Dinah M. Allen for helpful and stimulating discussions on the subject of barium stars. R.S. acknowledges a FAPERJ fellowship (E-26/150618/2000) during the development of this work, and later financial support from CAPES and FAPESP. G.F.P.M. acknowledges financial support from FAPERJ (grant APQ1/26/170687/2004), $\mathrm{CNPq} /$ Conteúdos Digitais (grant 552331/01-5) and from CNPq/Instituto do Milênio, 620053/2001-1. L. da S. thanks the CNPq, Brazilian Agency, for the grant 30137/86-7.

\section{References}

Antipova, L. I., Boyarchuk, A. A., Pakhomov, Yu. V., \& Panchuk, V. E. 2004, Astron. Rep., 48, 597

Allen, D. M., \& Barbuy, B. 2006, A\&A, 454, 895

Anders, E., \& Grevesse, N. 1989, Geochim. et Cosm. Acta, 53, 197

Bell, R. A., Eriksson, K., Gustafsson, B., \& Nordlund, A. 1976, A\&AS, 23, 37

Bergeat, J., \& Knapik, A. 1997, A\&A, 321, L9

Bidelman, W. P., \& Keenan, P. C. 1951, ApJ, 114, 473

Bisterzo, S., Gallino, R., Pignatari, M., et al. 2004, Mem. Soc. Astro. It., 75, 741

Blackwell, D. E., Lynas-Gray, A. E., \& Smith, G. 1995, A\&A, 296, 217

Blackwell, D. E., \& Lynas-Gray, A. E. 1998, A\&AS, 129, 505

Böhm-Vitense, E. 1980, ApJ, 239, L79

Böhm-Vitense, E., \& Johnson, H. R. 1985, ApJ, 293, 288

Böhm-Vitense, E., Nemec, J., \& Proffit, C. 1984, ApJ, 278, 726

Böhm-Vitense, E., Carpenter, K., Robinson, R., Ake, T., \& Brown, J. 2000, ApJ, 533, 969

Boyarchuk, A. A., Pakhomov, Yu. V., Antipova, L. I., \& Boyarchuk, M. E. 2002, Astron. Rep., 46, 819

Busso, M., Lambert, D. L., Beglio, L., et al. 1995, ApJ, 446, 775

Busso, M., Gallino, R., \& Wasserburg, G. J. 1999, ARA\&A, 37, 239

Busso, M., Gallino, R., Lambert, D. L., Travaglio, C., \& Smith, V. V. 2001, ApJ, 557,802
Burbidge, E. M., \& Burbidge, G. R. 1957, ApJ, 126, 357

Castro, S., Porto de Mello, G. F., \& da Silva, L. 1999, MNRAS, 305, 693

Cayrel de Strobel, G., Soubiran, C., \& Ralite, N. 2001, A\&A, 373, 159

Charbonnel, C., Meynet, G., Maeder, A., Schaller, G., \& Schaerer, D. 1993, A\&AS, 101, 415

Cowley, C. R., \& Downs, P. L. 1980, ApJ, 236, 648

Cunha, K., Smith, V. V., Suntzeff, N. B., et al. 2002, AJ, 124, 379

Dominy, J. F., \& Lambert, D. L. 1983, ApJ, 270, 180

ESA 1997, The Hipparcos and Tycho Catalogue, ESA SP-1200

Edvardsson, B., Andersen, J., Gustafsson, B., et al. 1993, A\&A, 275, 101

Hoffleit, D., \& Jaschek, C. 1982, The Bright Star Catalogue, Yale University Observatory, New Haven

Holweger, H., Kock, M., \& Bard, A. 1995, A\&A, 296, 233

Gallino, R., Arlandini, C., Busso, M., et al. 1998, ApJ, 497, 388

Griffin, R. F., \& Keenan, P. C. 1992, Obs., 112, 168.

Gómez, A. E., Luri, X., Grenier, S., et al. 1997, A\&A, 319, 881

Gustaffson, B., Bell, K. A., Eriksson, K. E., \& Nordlund, A. 1975, A\&A, 42, 407

Johnson, H. L., Mitchell, R. I., Iriarte, B., \& Wisniewski, W. Z. 1966, Comm. of the Lunar Planet. Lab., 4, 99

Jorissen, A., \& Mayor, M. 1992, A\&A, 260, 115

Jorissen, A., Van Eck, S., Mayor, M., \& Udry, S. 1998, A\&A, 332, 877

Kaufer, A., Stahl, O., Tubbesing, S., et al. 1999, The Messenger, 95, 8

Keenan, P. C., \& Pitts, R. E. 1980, ApJS, 71, 245

Kovacs, N. 1985, A\&A, 150, 232

Kurucz, R. L., Furelind, I., Brault, J., \& Testerman, L. 1984, The Solar Flux Atlas from $296 \mathrm{~nm}$ to $1300 \mathrm{~nm}$, National Solar Observatory

Landolt-Börnstein, 1982, New Series, Gp. VI, Vol 2, Subvolume B (Springer)

Liang, Y. C., Zhao, G., Chen, Y. Q., Qiu, H. M., \& Zhang, B. 2003, A\&A, 397, 257

Liang, Y. C., Zhao, G., \& Zhang, B. 2000, A\&A, 363, 555

Lu, P. K. 1991, AJ, 101, 2229

Luck, R. E., \& Bond, H. E. 1991, ApJS, 77, 515

Matteucci, F., Raiteri, C. M., Busso, M., Gallino, R., \& Gratton, R. 1993, A\&A, 272,421

Malaney, R. A. 1987a, ApJ, 321, 832

Malaney, R. A. 1987b, Ap\&SS, 137, 251

McClure, R. D. 1983, ApJ, 268, 264

McClure, R. D. 1984, PASP, 96, 117

McClure, R. D., Fletcher, J. M., \& Nemec, J. M. 1980, ApJ, 238, L35

McWilliam, A. 1990, ApJS, 74, 1075

McWilliam, A. 1997, ARA\&A, 35, 503

McWilliam, A., Rich, R. M., \& Smecker-Hane, T. A. 2003, ApJ, 592, L21

McWilliam, A., \& Smecker-Hane, T. A. 2005, ApJ, 622, L29

Mishenina, T. V., Kovtyukh, V. V., Soubiran, C., Travaglio, C., \& Busso, M. 2002, A\&A, 396, 189

Pereira, C. B., \& Porto de Mello, G. F. 1997, AJ, 114, 2128

Pereira, C. B., Smith, V., \& Cunha, K. M. L. 1998, AJ, 116, 1977

Pilachowski, C. A. 1977, A\&A, 54, 465

Plez, B., Brett, J. M., \& Nordlund, A. 1992, A\&A, 256, 551

Porto de Mello, G., \& da Silva, L. 1997, ApJ, 476, L89

Schaller, G., Schaerer, D., Meynet, G., \& Maeder, M. 1992, A\&AS, 96, 269

Schaerer, D., Meynet, G., Maeder, A., \& Schaller, G. 1993, A\&AS, 98, 253

Simmerer, J., Sneden, C., Ivans, I. I., et al. 2003, AJ, 125, 2018

Smith, V. V. 1984, A\&A, 132, 326

Smith, V. V., \& Lambert, D. L. 1987, MNRAS, 226, 563

Sneden, C., Gratton, R., \& Crocker, D. 1991, A\&A, 246, 354

Soderblom, D., \& Mayor, M. 1993, AJ, 105, 226

Steffen, M. 1985, A\&AS, 59, 403

Tomkin, J., \& Lambert, D. L. 1979, ApJ, 227, 209

Tomkin, J., \& Lambert, D. L. 1983, ApJ, 273, 722

Travaglio, C., Gallino, R., Busso, M., \& Gratton, R. 2001, ApJ, 549, 346

Udry, S., Jorissen, A., Mayor, M., \& Van Eck, S. 1998a, A\&AS, 131, 25

Udry, S., Mayor, M., Van Eck, S., et al. 1998b, A\&AS, 131, 43

Warner, B. 1965, MNRAS, 129, 263

Yushchenko, A. V., Gopka, V. F., Kim, C., et al. 2004, A\&A, 413, 1105

Zacs, L. 1994, A\&A, 283, 93 
R. Smiljanic et al.: Abundance analysis of barium and mild barium stars, Online Material $p 1$

\section{Online Material}


R. Smiljanic et al.: Abundance analysis of barium and mild barium stars, Online Material p 2

Table 8. Equivalent widths for the stars HR 440, HR 649, HR 1016, HR 1326, HR 2392 and HR 4608. The derived log $g f$ s are also listed.

\begin{tabular}{|c|c|c|c|c|c|c|c|c|c|}
\hline$\lambda(\AA)$ & Elem. & $\chi(\mathrm{eV})$ & $\log g f$ & HR 440 & HR 649 & HR 1016 & HR 1326 & HR 2392 & HR 4608 \\
\hline 6154.230 & $\mathrm{NaI}$ & 2.10 & $\begin{array}{l}-1.179 \\
\end{array}$ & 60.4 & 75.5 & 73.5 & 89.1 & 81.9 & 55.5 \\
\hline 6160.753 & $\mathrm{NaI}$ & 2.10 & -0.905 & 83.8 & 98.2 & 92.7 & 107.1 & 105.4 & 76.6 \\
\hline 4571.102 & $\mathrm{MgI}$ & 0.00 & - & 194.5 & - & 193.6 & - & 259.6 & 186.8 \\
\hline 4730.038 & $\mathrm{MgI}$ & 4.34 & - & 107.5 & 104.8 & 108.5 & 126.2 & 141.3 & 105.2 \\
\hline 5711.095 & $\mathrm{MgI}$ & 4.34 & - & 135.1 & 133.7 & 127.6 & 148.2 & 142.8 & 125.0 \\
\hline 5785.285 & $\mathrm{MgI}$ & 5.11 & -1.615 & 76.9 & 78.4 & 76.5 & 91.6 & 87.0 & 62.9 \\
\hline 6696.032 & AlI & 3.14 & -1.370 & 66.7 & 58.4 & 58.4 & 91.5 & 67.1 & 56.3 \\
\hline 6698.669 & AlI & 3.14 & -1.659 & 43.9 & 38.3 & 40.0 & 69.1 & 65.2 & 38.4 \\
\hline 5517.533 & SiI & 5.08 & -2.240 & 30.3 & 33.8 & - & 79.7 & 27.8 & 20.4 \\
\hline 5665.563 & SiI & 4.92 & -1.590 & 75.9 & 79.3 & 72.1 & 73.2 & - & - \\
\hline 5684.484 & SiI & 4.95 & -1.420 & 81.8 & 89.9 & 84.7 & 59.9 & 88.3 & 75.8 \\
\hline 5690.433 & SiI & 4.93 & -1.576 & 76.9 & 85.9 & 76.2 & - & 96.2 & 67.3 \\
\hline 5701.108 & SiI & 4.93 & -1.817 & 61.5 & 65.8 & 61.7 & - & 68.3 & 54.9 \\
\hline 5708.405 & SiI & 4.95 & -1.098 & 104.0 & 117.5 & 108.2 & - & - & - \\
\hline 5753.622 & SiI & 5.61 & -0.883 & 63.3 & 71.1 & 68.1 & - & - & - \\
\hline 5772.149 & SiI & 5.08 & -1.299 & 72.9 & 81.9 & 81.0 & - & - & - \\
\hline 5793.080 & SiI & 4.93 & -1.778 & 61.3 & 66.5 & 61.7 & 65.2 & 81.5 & 51.9 \\
\hline 6131.577 & SiI & 5.61 & -1.505 & 35.1 & 38.8 & 40.8 & 40.3 & 42.2 & 27.0 \\
\hline 6131.858 & SiI & 5.61 & -1.521 & 35.8 & 41.3 & 41.9 & 40.8 & - & - \\
\hline 6142.494 & SiI & 5.62 & -1.304 & 44.3 & 50.1 & 51.7 & 45.5 & - & 43.2 \\
\hline 6145.020 & SiI & 5.61 & -1.272 & 45.7 & 51.0 & 53.5 & 49.3 & 55.1 & 43.2 \\
\hline 6243.823 & SiI & 5.61 & -1.067 & 52.0 & 57.8 & 74.5 & 72.1 & - & 54.3 \\
\hline 6721.844 & SiI & 5.86 & -0.827 & 55.5 & 62.1 & 61.8 & 65.6 & 83.2 & 51.1 \\
\hline 5261.708 & $\mathrm{CaI}$ & 2.52 & -0.383 & 134.4 & 139.0 & 135.1 & - & 138.2 & 122.3 \\
\hline 5581.979 & $\mathrm{CaI}$ & 2.52 & -0.539 & 129.4 & 126.6 & 123.4 & 145.0 & - & 120.6 \\
\hline 5590.126 & $\mathrm{CaI}$ & 2.52 & -0.724 & 120.9 & 122.7 & 115.9 & 131.6 & 118.1 & 110.4 \\
\hline 5867.572 & $\mathrm{CaI}$ & 2.93 & -1.503 & 44.2 & 42.9 & 45.8 & 68.2 & 53.9 & 37.0 \\
\hline 6161.295 & $\mathrm{CaI}$ & 2.52 & -1.027 & 99.4 & 99.0 & 100.4 & 123.7 & - & 100.8 \\
\hline 6163.754 & $\mathrm{CaI}$ & 2.52 & -1.290 & 79.8 & 87.0 & - & 96.9 & 92.7 & 75.6 \\
\hline 6166.440 & $\mathrm{CaI}$ & 2.52 & -1.097 & 98.7 & 97.9 & 98.1 & 115.6 & 115.1 & 95.5 \\
\hline 6169.044 & $\mathrm{CaI}$ & 2.52 & -0.618 & 118.6 & 119.3 & 122.4 & 141.5 & 136.6 & 117.1 \\
\hline 6449.820 & $\mathrm{CaI}$ & 2.52 & -0.565 & 128.8 & 136.0 & 130.3 & 138.7 & 137.2 & 124.5 \\
\hline 6455.605 & $\mathrm{CaI}$ & 2.52 & -1.168 & 87.8 & 87.3 & - & 112.5 & 109.9 & 91.1 \\
\hline 6499.654 & $\mathrm{CaI}$ & 2.52 & -0.706 & 120.6 & 122.1 & 119.2 & 138.6 & 135.1 & 112.3 \\
\hline 6798.467 & $\mathrm{CaI}$ & 2.71 & -2.087 & 20.5 & - & - & 49.5 & 38.8 & - \\
\hline 5318.346 & ScII & 1.36 & - & 41.2 & 48.6 & 45.3 & 43.0 & 47.6 & 38.2 \\
\hline 5357.190 & ScII & 1.51 & - & 22.4 & 22.8 & 18.1 & 19.9 & 21.4 & 17.0 \\
\hline 5526.815 & ScII & 1.77 & - & 119.2 & 132.6 & 125.0 & 118.9 & 132.0 & 115.2 \\
\hline 5657.874 & ScII & 1.51 & - & 122.7 & 138.2 & 119.2 & 113.3 & 130.6 & 114.9 \\
\hline 5684.189 & ScII & 1.51 & - & 87.8 & 97.0 & 83.1 & 85.3 & 97.0 & 81.0 \\
\hline 6245.660 & ScII & 1.51 & - & 76.9 & 85.4 & 87.6 & 88.9 & 87.1 & 79.9 \\
\hline 6320.867 & ScII & 1.50 & - & 36.3 & 42.7 & 37.2 & 38.8 & 43.5 & 38.2 \\
\hline 4518.023 & TiI & 0.83 & -0.401 & 125.3 & 121.7 & 114.6 & 141.5 & 137.7 & 115.9 \\
\hline 4548.765 & TiI & 0.83 & -0.396 & 122.5 & 119.6 & 115.7 & 144.4 & 143.4 & 117.2 \\
\hline 4555.485 & TiI & 0.35 & -1.067 & - & 114.7 & 111.5 & 137.8 & - & - \\
\hline 4562.625 & TiI & 0.02 & -2.638 & 65.2 & 45.9 & 48.3 & 90.5 & 70.1 & 52.0 \\
\hline 4617.254 & TiI & 1.75 & 0.146 & 101.4 & 98.8 & - & 118.4 & 108.3 & 94.2 \\
\hline 4758.120 & TiI & 2.25 & 0.253 & 80.7 & 75.9 & 75.9 & 98.2 & 102.4 & 77.1 \\
\hline 4759.272 & TiI & 2.25 & 0.273 & 84.7 & 79.6 & 78.1 & 100.3 & - & 80.6 \\
\hline 4778.259 & TiI & 2.24 & -0.359 & 51.9 & 39.4 & 44.3 & 78.9 & 53.1 & 41.7 \\
\hline 4926.147 & TiI & 0.82 & -2.157 & 41.4 & 26.4 & 26.3 & 69.4 & 45.7 & 30.1 \\
\hline 5022.871 & TiI & 0.83 & -0.427 & 133.3 & 127.9 & 123.9 & - & 134.6 & 119.0 \\
\hline 5024.842 & TiI & 0.82 & -0.580 & 123.7 & 118.6 & 115.8 & 144.4 & 116.3 & 111.7 \\
\hline 5071.472 & TiI & 1.46 & -0.598 & - & - & 70.7 & 112.6 & 84.5 & 69.8 \\
\hline 5113.448 & TiI & 1.44 & -0.826 & 74.6 & 61.8 & 64.8 & 103.8 & 65.1 & 66.6 \\
\hline 5145.464 & TiI & 1.46 & -0.634 & 87.5 & 74.6 & 75.4 & 109.0 & 89.4 & 77.7 \\
\hline 5147.479 & TiI & 0.00 & -1.927 & 108.9 & 97.5 & 95.4 & 135.4 & - & - \\
\hline 5152.185 & TiI & 0.02 & -2.185 & 97.1 & 86.8 & 84.1 & 118.1 & 102.4 & 90.4 \\
\hline 5211.206 & TiI & 0.84 & -2.146 & - & 22.7 & 27.0 & - & 28.6 & 25.2 \\
\hline 5219.700 & TiI & 0.02 & -2.174 & 95.6 & 79.5 & 79.6 & 121.5 & 106.4 & 86.1 \\
\hline 5295.780 & TiI & 1.07 & -1.622 & 55.1 & 42.4 & 43.2 & 81.4 & 53.8 & 44.5 \\
\hline 5426.236 & TiI & 0.02 & -2.912 & 51.7 & 31.2 & 32.4 & 85.2 & 63.9 & 41.9 \\
\hline 5471.197 & TiI & 1.44 & -1.347 & - & 29.4 & 43.9 & 80.3 & 44.0 & 35.3 \\
\hline 5490.150 & TiI & 1.46 & -0.834 & 72.5 & 62.3 & 66.9 & 99.6 & 73.8 & 63.6 \\
\hline 5648.567 & TiI & 2.49 & -0.210 & 42.8 & 38.3 & 28.4 & 61.2 & 56.5 & 32.2 \\
\hline 5739.464 & TiI & 2.25 & -0.703 & - & - & 21.3 & 53.7 & 35.3 & 22.0 \\
\hline
\end{tabular}


R. Smiljanic et al.: Abundance analysis of barium and mild barium stars, Online Material p 3

Table 8. continued.

\begin{tabular}{|c|c|c|c|c|c|c|c|c|c|}
\hline$\lambda(\AA)$ & Elem. & $x(\mathrm{eV})$ & $\log g f$ & HR 440 & HR 649 & HR 1016 & HR 1326 & HR 2392 & HR 4608 \\
\hline 5866.452 & TiI & 1.07 & -0.693 & 109.1 & 95.8 & 94.9 & 137.7 & 118.3 & 96.4 \\
\hline 6064.629 & TiI & 1.05 & -1.709 & - & 38.0 & 35.2 & 79.0 & 66.9 & 38.7 \\
\hline 6126.224 & TiI & 1.07 & -1.322 & 80.5 & 68.0 & 68.4 & 105.6 & - & 74.2 \\
\hline 6258.104 & TiI & 1.44 & -0.326 & - & - & - & 126.1 & 107.8 & 96.6 \\
\hline 6861.500 & TiI & 2.27 & -0.667 & 30.8 & 29.1 & 25.1 & 55.1 & 45.3 & 23.4 \\
\hline 4524.691 & TilI & 1.23 & -2.892 & 69.7 & 75.9 & 70.5 & 72.5 & - & 70.8 \\
\hline 4568.345 & TilI & 1.22 & -2.710 & 78.0 & 83.8 & 82.3 & - & 82.0 & 73.7 \\
\hline 4583.415 & TilI & 1.16 & -2.769 & 78.6 & 87.7 & 83.7 & 78.1 & 86.4 & 73.1 \\
\hline 4657.209 & TilI & 1.24 & -2.144 & - & 110.4 & 105.3 & 104.7 & 116.6 & 95.5 \\
\hline 4798.539 & TiII & 1.08 & -2.785 & 88.6 & 95.1 & 86.7 & 88.0 & 87.0 & 84.2 \\
\hline 5211.544 & TilI & 2.59 & -1.481 & 61.9 & - & 67.8 & 58.7 & 61.8 & 62.6 \\
\hline 5336.783 & TiII & 1.58 & -1.697 & 115.0 & 129.5 & - & 110.1 & 125.4 & 110.8 \\
\hline 5381.020 & TilI & 1.57 & -1.598 & 121.3 & 132.2 & 115.0 & - & _- & 116.9 \\
\hline 5418.756 & TilI & 1.58 & -2.183 & 87.4 & 97.7 & 88.2 & 83.4 & 92.8 & 85.9 \\
\hline 5657.436 & VI & 1.06 & - & 50.2 & 39.4 & 30.6 & 75.1 & 59.1 & 31.9 \\
\hline 5668.362 & VI & 1.08 & - & 46.6 & 35.7 & 28.5 & 74.6 & 48.2 & 31.3 \\
\hline 5670.851 & VI & 1.08 & - & 87.3 & 67.3 & 62.4 & 122.7 & 89.3 & 68.5 \\
\hline 5727.661 & VI & 1.05 & - & 57.6 & 40.9 & 34.3 & 95.2 & 53.2 & 35.9 \\
\hline 6090.216 & VI & 1.08 & - & 90.0 & 79.3 & 74.6 & 113.3 & 100.6 & - \\
\hline 6135.370 & VI & 1.05 & - & 56.1 & 40.0 & 42.2 & 92.7 & 67.8 & 40.6 \\
\hline 6150.154 & VI & 0.30 & - & 66.3 & 46.1 & 48.5 & 115.9 & 73.7 & 50.3 \\
\hline 6199.186 & VI & 0.29 & - & 77.7 & 48.9 & 54.5 & 130.9 & 81.6 & 58.8 \\
\hline 6216.358 & VI & 0.28 & - & 103.6 & 82.5 & 85.2 & - & - & 79.1 \\
\hline 6274.658 & VI & 0.27 & - & 54.3 & 35.9 & 35.8 & 97.0 & 71.0 & 40.0 \\
\hline 6285.165 & VI & 0.28 & - & 62.0 & 43.7 & 44.0 & 99.8 & 64.4 & - \\
\hline 4616.120 & $\mathrm{CrI}$ & 0.98 & -1.344 & 135.2 & 134.8 & - & - & - & 129.5 \\
\hline 4626.174 & $\mathrm{CrI}$ & 0.97 & -1.534 & 124.9 & 130.1 & - & 144.6 & 140.5 & 116.1 \\
\hline 4708.019 & $\mathrm{CrI}$ & 3.17 & 0.191 & 89.5 & 97.3 & 96.9 & 112.0 & - & - \\
\hline 4737.355 & $\mathrm{CrI}$ & 3.09 & 0.270 & 94.1 & 105.7 & 102.0 & 114.3 & - & - \\
\hline 4756.137 & $\mathrm{CrI}$ & 3.10 & 0.159 & 92.2 & 94.8 & 94.8 & 108.4 & - & 91.3 \\
\hline 4801.047 & $\mathrm{CrI}$ & 3.12 & -0.124 & 81.4 & 87.8 & 84.6 & 99.8 & - & - \\
\hline 4936.335 & $\mathrm{CrI}$ & 3.11 & -0.172 & 78.2 & 74.9 & 74.9 & 98.1 & - & 73.3 \\
\hline 4964.916 & $\mathrm{CrI}$ & 0.94 & -2.615 & 82.7 & 76.7 & 76.2 & 102.1 & 76.2 & 72.9 \\
\hline 5200.207 & $\mathrm{CrI}$ & 3.38 & -0.346 & 51.0 & 57.3 & 53.4 & 74.4 & - & - \\
\hline 5214.144 & $\mathrm{CrI}$ & 3.37 & -0.623 & 33.6 & 32.0 & 34.0 & 50.7 & 31.5 & 26.4 \\
\hline 5238.964 & $\mathrm{CrI}$ & 2.71 & -1.183 & 44.1 & 40.7 & 39.0 & - & 40.5 & 39.2 \\
\hline 5247.566 & $\mathrm{CrI}$ & 0.96 & -1.605 & 135.0 & 131.9 & 125.6 & - & 132.3 & 123.0 \\
\hline 5272.007 & $\mathrm{CrI}$ & 3.45 & -0.301 & 47.1 & 46.7 & 49.2 & 71.5 & 66.1 & 41.5 \\
\hline 5287.183 & $\mathrm{CrI}$ & 3.44 & -0.751 & 25.9 & 27.5 & 27.1 & 44.7 & - & - \\
\hline 5300.751 & $\mathrm{CrI}$ & 0.98 & -2.044 & 107.7 & 101.9 & 100.2 & 126.4 & 113.7 & 99.0 \\
\hline 5304.183 & $\mathrm{CrI}$ & 3.46 & -0.613 & 28.1 & 28.5 & 29.9 & 48.4 & 37.0 & 23.4 \\
\hline 5318.810 & $\mathrm{CrI}$ & 3.44 & -0.590 & 30.1 & 30.3 & 33.8 & 51.8 & - & 23.9 \\
\hline 5628.621 & $\mathrm{CrI}$ & 3.42 & -0.534 & - & 34.2 & - & - & 37.6 & 33.4 \\
\hline 5784.976 & $\mathrm{CrI}$ & 3.32 & -0.326 & 60.0 & 58.3 & 57.2 & 79.5 & 69.2 & 44.4 \\
\hline 5787.965 & $\mathrm{CrI}$ & 3.32 & -0.105 & 75.4 & 77.7 & 72.2 & 91.7 & 86.5 & 63.1 \\
\hline 6330.097 & $\mathrm{CrI}$ & 0.94 & -2.784 & - & 69.4 & 68.6 & 102.9 & 91.9 & 69.7 \\
\hline 4588.203 & CrII & 4.07 & -0.639 & 90.3 & 111.0 & 108.6 & 91.7 & - & 89.6 \\
\hline 4592.049 & CrII & 4.07 & -1.135 & 66.5 & 79.7 & 79.7 & 70.6 & 86.9 & 64.8 \\
\hline 5305.855 & CrII & 3.83 & -1.882 & 39.4 & 57.1 & 51.6 & 40.0 & 56.5 & 40.8 \\
\hline 5308.377 & CrII & 4.07 & -1.706 & 39.1 & 50.5 & 49.2 & 47.7 & 51.3 & 38.4 \\
\hline 5313.526 & CrII & 4.07 & -1.433 & 48.2 & 68.7 & 62.8 & 48.0 & - & 55.5 \\
\hline 5502.025 & CrII & 4.17 & -1.653 & 33.8 & 47.1 & 54.1 & - & 62.9 & 30.7 \\
\hline 4739.113 & MnI & 2.94 & - & 94.8 & 91.7 & 93.9 & 112.0 & 116.2 & 92.1 \\
\hline 5413.684 & MnI & 3.86 & - & 43.9 & 37.6 & 42.1 & 77.3 & 58.8 & 33.5 \\
\hline 5420.350 & MnI & 2.14 & - & 175.9 & 148.4 & 156.3 & 220.5 & - & 147.1 \\
\hline 5432.548 & MnI & 0.00 & - & - & - & - & 192.7 & - & 129.8 \\
\hline 5537.765 & MnI & 2.19 & - & 100.9 & 79.9 & 90.8 & - & 121.7 & - \\
\hline 6013.497 & MnI & 3.07 & - & 125.4 & 122.5 & 122.9 & - & 138.0 & 115.2 \\
\hline 6021.803 & MnI & 3.07 & - & 129.7 & 132.7 & 127.9 & - & 140.8 & 118.4 \\
\hline 4523.400 & FeI & 3.65 & -1.860 & 71.6 & 74.6 & 74.5 & 84.3 & - & 67.8 \\
\hline 4537.676 & $\mathrm{FeI}$ & 3.27 & -2.862 & 40.6 & 36.3 & 41.6 & 63.6 & - & 33.2 \\
\hline 4556.925 & $\mathrm{FeI}$ & 3.25 & -2.585 & 56.8 & 53.9 & 56.9 & 72.5 & - & 55.4 \\
\hline 4585.343 & FeI & 4.61 & -1.438 & 34.2 & 38.0 & 45.7 & 54.5 & 46.3 & 27.7 \\
\hline 4593.555 & $\mathrm{FeI}$ & 3.94 & -1.821 & 60.8 & - & - & 75.8 & - & - \\
\hline 4598.125 & FeI & 3.28 & -1.422 & 110.3 & 113.2 & 113.4 & 120.6 & 115.4 & 101.8 \\
\hline 4602.000 & FeI & 1.61 & -3.148 & 120.0 & 120.9 & 120.4 & 133.5 & - & 113.2 \\
\hline 4741.535 & FeI & 2.83 & -2.084 & 109.6 & - & 109.8 & 118.5 & - & - \\
\hline
\end{tabular}


R. Smiljanic et al.: Abundance analysis of barium and mild barium stars, Online Material p 4

Table 8. continued.

\begin{tabular}{|c|c|c|c|c|c|c|c|c|c|}
\hline$\lambda(\AA)$ & Elem. & $x(\mathrm{eV})$ & $\log g f$ & HR 440 & HR 649 & HR 1016 & HR 1326 & HR 2392 & HR 4608 \\
\hline 4749.961 & $\mathrm{FeI}$ & 4.56 & -1.177 & 56.6 & 57.7 & 58.0 & 69.0 & - & - \\
\hline 4793.961 & FeI & 3.05 & -3.467 & 31.5 & 25.2 & 25.4 & 46.5 & 39.9 & - \\
\hline 4794.355 & FeI & 2.42 & -3.906 & 44.4 & 35.0 & 37.1 & 62.5 & 44.1 & 35.7 \\
\hline 4798.273 & FeI & 4.19 & -1.442 & 70.0 & 69.9 & 70.8 & 76.8 & 70.9 & 61.3 \\
\hline 4798.743 & $\mathrm{FeI}$ & 1.61 & -4.163 & 83.4 & 77.7 & 77.0 & 94.2 & 84.0 & 74.3 \\
\hline 4808.147 & FeI & 3.25 & -2.614 & 61.8 & 60.1 & 60.0 & 73.2 & 57.6 & 49.2 \\
\hline 4907.733 & $\mathrm{FeI}$ & 3.43 & -1.640 & 97.0 & 99.1 & 96.3 & 109.9 & 99.0 & 86.4 \\
\hline 4908.032 & $\mathrm{FeI}$ & 3.93 & -1.552 & 69.1 & 69.6 & 70.2 & 89.2 & 74.1 & - \\
\hline 4911.788 & FeI & 3.93 & -1.712 & 67.6 & 67.1 & 67.3 & 77.9 & 61.5 & 58.9 \\
\hline 4961.915 & FeI & 3.63 & -2.135 & 59.1 & 59.9 & 59.2 & 74.6 & - & - \\
\hline 4962.565 & FeI & 4.18 & -1.191 & 75.7 & 80.5 & 77.7 & 87.9 & 81.7 & 71.5 \\
\hline 4969.916 & FeI & 4.22 & -0.675 & 98.7 & 101.8 & 101.7 & 115.2 & 114.7 & 94.7 \\
\hline 5023.189 & FeI & 4.28 & -1.324 & 66.6 & 65.9 & 70.7 & _- & 66.8 & 55.0 \\
\hline 5025.091 & FeI & 4.26 & -1.836 & 38.9 & 40.6 & 40.9 & 53.6 & 52.1 & 32.3 \\
\hline 5025.313 & $\mathrm{FeI}$ & 4.28 & -1.849 & 38.3 & 36.4 & 39.7 & 49.4 & 54.5 & 32.8 \\
\hline 5054.647 & $\mathrm{FeI}$ & 3.64 & -1.931 & 69.0 & 66.9 & 68.1 & 89.2 & 88.5 & 66.9 \\
\hline 5067.162 & FeI & 4.22 & -0.668 & 100.8 & 100.6 & 99.0 & 116.1 & 116.2 & 96.8 \\
\hline 5072.677 & FeI & 4.22 & -0.892 & 92.8 & 91.3 & 90.4 & - & 101.6 & 85.2 \\
\hline 5109.649 & FeI & 4.30 & -0.582 & 100.3 & 104.1 & - & - & 105.5 & 93.9 \\
\hline 5127.680 & FeI & 0.05 & -5.908 & 94.2 & 75.7 & 77.7 & - & 99.3 & 79.7 \\
\hline 5196.065 & $\mathrm{FeI}$ & 4.26 & -0.809 & 96.6 & 99.5 & 97.5 & - & 102.1 & 90.0 \\
\hline 5197.929 & FeI & 4.30 & -1.417 & 57.9 & 60.7 & 61.8 & 71.4 & 69.5 & 52.1 \\
\hline 5213.818 & FeI & 3.94 & -2.508 & 21.2 & 19.9 & 21.1 & 35.4 & 22.7 & 18.5 \\
\hline 5223.188 & $\mathrm{FeI}$ & 3.63 & -2.215 & 53.5 & 53.9 & 59.9 & 71.4 & 54.7 & 51.4 \\
\hline 5242.491 & $\mathrm{FeI}$ & 3.63 & -0.983 & 118.0 & 122.7 & 114.7 & - & 118.5 & 118.1 \\
\hline 5243.773 & FeI & 4.26 & -0.915 & 88.3 & 93.2 & 87.8 & 96.2 & 90.2 & 85.1 \\
\hline 5247.049 & FeI & 0.09 & -4.743 & 146.7 & 136.1 & 127.8 & - & 142.6 & 130.3 \\
\hline 5320.040 & $\mathrm{FeI}$ & 3.64 & -2.496 & 45.2 & 44.8 & 44.2 & 58.9 & 46.8 & 33.5 \\
\hline 5321.109 & FeI & 4.43 & -1.237 & 65.4 & 66.6 & 68.6 & 75.1 & - & 57.9 \\
\hline 5379.574 & FeI & 3.69 & -1.549 & 96.0 & 97.0 & 90.0 & 98.8 & 109.5 & 82.0 \\
\hline 5389.486 & FeI & 4.41 & -0.567 & 103.6 & - & 103.9 & 110.5 & 110.7 & 97.0 \\
\hline 5395.222 & FeI & 4.44 & -1.656 & 36.7 & - & 36.8 & 52.1 & 48.2 & 29.1 \\
\hline 5412.791 & FeI & 4.43 & -1.618 & 38.7 & 38.4 & 37.9 & 53.6 & 45.9 & 30.2 \\
\hline 5432.946 & $\mathrm{FeI}$ & 4.44 & -0.639 & 87.1 & 95.6 & 98.0 & 99.2 & 93.7 & 84.7 \\
\hline 5436.297 & FeI & 4.39 & -1.185 & 59.3 & - & 65.3 & - & 62.5 & - \\
\hline 5473.168 & FeI & 4.19 & -1.846 & 35.9 & 43.0 & - & 59.8 & - & - \\
\hline 5483.108 & FeI & 4.15 & -1.366 & 69.1 & 73.1 & - & 86.0 & 83.0 & 68.5 \\
\hline 5491.845 & FeI & 4.19 & -1.938 & 31.9 & 31.7 & 40.1 & 53.2 & 42.9 & 30.1 \\
\hline 5494.474 & FeI & 4.07 & -1.645 & 54.7 & 59.3 & 63.9 & 76.9 & 60.0 & 51.4 \\
\hline 5508.419 & FeI & 4.96 & -1.233 & 32.5 & 34.0 & - & 45.4 & - & - \\
\hline 5522.454 & FeI & 4.21 & -1.428 & 68.3 & 69.9 & - & 82.0 & 74.5 & 58.3 \\
\hline 5560.207 & FeI & 4.43 & -1.020 & 73.8 & 74.6 & 74.3 & - & 76.9 & 64.5 \\
\hline 5577.013 & FeI & 5.03 & -1.285 & 24.0 & 21.7 & 22.5 & 29.5 & 27.6 & 17.2 \\
\hline 5587.573 & $\mathrm{FeI}$ & 4.14 & -1.538 & 65.1 & 64.3 & 62.0 & 73.9 & 68.6 & 53.3 \\
\hline 5635.824 & FeI & 4.26 & -1.364 & 63.8 & 65.9 & - & 73.0 & 64.1 & 59.9 \\
\hline 5636.705 & FeI & 3.64 & -2.260 & 53.4 & 53.7 & - & 63.6 & 60.6 & 46.9 \\
\hline 5638.262 & FeI & 4.22 & -0.606 & 112.9 & - & 105.8 & 116.6 & 130.6 & 101.1 \\
\hline 5641.436 & $\mathrm{FeI}$ & 4.26 & -0.619 & 107.8 & 110.5 & - & 114.8 & 112.9 & 93.0 \\
\hline 5646.697 & FeI & 4.26 & -2.047 & - & - & 22.7 & - & 45.0 & 22.1 \\
\hline 5650.019 & $\mathrm{FeI}$ & 5.10 & -0.520 & 62.1 & 65.9 & 58.6 & 68.6 & 81.6 & 50.7 \\
\hline 5652.319 & $\mathrm{FeI}$ & 4.26 & -1.541 & 58.1 & 59.3 & 51.4 & 64.3 & 65.4 & 47.2 \\
\hline 5661.348 & $\mathrm{FeI}$ & 4.28 & -1.526 & 57.8 & 58.1 & 51.1 & 66.5 & - & 46.1 \\
\hline 5680.240 & FeI & 4.19 & -1.958 & 37.7 & 37.9 & 33.8 & 48.2 & - & 35.8 \\
\hline 5701.557 & FeI & 2.56 & -1.943 & - & 136.5 & 127.7 & 149.5 & 145.6 & 124.4 \\
\hline 5705.473 & $\mathrm{FeI}$ & 4.30 & -1.322 & 64.9 & 66.8 & 65.1 & 75.9 & 68.7 & 57.4 \\
\hline 5731.761 & FeI & 4.26 & -1.063 & 88.9 & - & 86.4 & 93.6 & 103.3 & 77.2 \\
\hline 5738.240 & FeI & 4.22 & -2.048 & - & - & 27.0 & 44.9 & 34.7 & - \\
\hline 5775.069 & $\mathrm{FeI}$ & 4.22 & -1.047 & 85.2 & 88.8 & 86.7 & 94.8 & 85.3 & 82.0 \\
\hline 5778.463 & FeI & 2.59 & -3.331 & 63.1 & 57.5 & 57.2 & 78.2 & 64.3 & 60.5 \\
\hline 5784.666 & FeI & 3.40 & -2.544 & 63.4 & - & 58.3 & 75.3 & - & 46.7 \\
\hline 5811.916 & $\mathrm{FeI}$ & 4.14 & -2.262 & 27.1 & 26.9 & 26.5 & 38.5 & 31.0 & 19.5 \\
\hline 5814.805 & $\mathrm{FeI}$ & 4.28 & -1.730 & 46.0 & 46.3 & 44.8 & 59.0 & 58.1 & 37.2 \\
\hline 5835.098 & $\mathrm{FeI}$ & 4.26 & -1.911 & 32.1 & 32.3 & 33.5 & 47.4 & 47.7 & 29.1 \\
\hline 5849.681 & $\mathrm{FeI}$ & 3.69 & -2.776 & 21.5 & 19.2 & 20.1 & 36.0 & 32.5 & 21.5 \\
\hline 5852.222 & FeI & 4.55 & -0.968 & 63.8 & - & 66.0 & - & 70.2 & 60.2 \\
\hline 5855.086 & FeI & 4.61 & -1.381 & 35.8 & 36.9 & 40.0 & 52.2 & 55.7 & 33.8 \\
\hline 5856.096 & FeI & 4.29 & -1.410 & 54.1 & 56.5 & 57.8 & 70.1 & 79.9 & 52.8 \\
\hline
\end{tabular}


R. Smiljanic et al.: Abundance analysis of barium and mild barium stars, Online Material p 5

Table 8. continued.

\begin{tabular}{|c|c|c|c|c|c|c|c|c|c|}
\hline$\lambda(\AA)$ & Elem. & $\chi(\mathrm{eV})$ & $\log g f$ & HR 440 & HR 649 & HR 1016 & HR 1326 & HR 2392 & HR 4608 \\
\hline 5859.596 & $\mathrm{FeI}$ & 4.55 & -0.536 & 92.1 & 97.8 & 97.4 & 103.3 & 112.2 & 88.0 \\
\hline 6005.551 & FeI & 2.59 & -3.271 & - & 60.3 & - & 80.3 & 78.5 & 55.1 \\
\hline 6007.968 & FeI & 4.65 & -0.602 & 76.8 & 88.6 & 82.3 & 90.8 & 94.8 & 74.9 \\
\hline 6012.212 & FeI & 2.22 & -3.692 & - & 58.8 & - & 82.8 & 71.0 & - \\
\hline 6078.499 & FeI & 4.79 & -0.150 & 91.0 & 100.4 & - & - & 101.0 & - \\
\hline 6079.014 & FeI & 4.65 & -0.847 & 64.2 & 70.2 & - & - & 69.9 & - \\
\hline 6093.666 & FeI & 4.61 & -1.185 & 52.5 & 56.6 & 53.0 & 61.0 & 56.6 & - \\
\hline 6098.250 & FeI & 4.56 & -1.515 & 38.8 & - & 39.4 & 47.3 & - & 36.0 \\
\hline 6120.249 & FeI & 0.92 & -5.730 & 42.6 & - & 31.9 & 62.2 & 49.9 & 29.9 \\
\hline 6137.002 & $\mathrm{FeI}$ & 2.20 & -2.857 & 114.8 & 113.8 & 112.3 & 129.4 & 123.9 & 106.3 \\
\hline 6151.616 & FeI & 2.18 & -3.259 & 96.2 & 95.1 & 92.8 & 109.2 & 103.0 & 91.5 \\
\hline 6159.382 & FeI & 4.61 & -1.666 & 26.5 & 25.8 & 28.6 & 41.1 & - & 26.6 \\
\hline 6173.340 & FeI & 2.22 & -2.739 & 116.6 & 115.8 & 113.3 & 129.7 & 137.1 & 112.1 \\
\hline 6187.987 & FeI & 3.94 & -1.620 & 72.7 & 75.3 & 72.8 & 85.8 & 89.1 & 69.6 \\
\hline 6199.508 & FeI & 2.56 & -4.134 & 20.4 & 17.6 & 18.8 & 36.4 & 34.5 & 16.8 \\
\hline 6200.321 & FeI & 2.61 & -2.290 & 119.0 & 119.3 & 115.7 & 133.7 & 125.4 & 108.3 \\
\hline 6213.428 & $\mathrm{FeI}$ & 2.22 & -2.479 & 136.0 & 135.7 & 133.2 & - & 143.7 & 122.3 \\
\hline 6226.730 & FeI & 3.88 & -1.940 & 53.3 & 54.7 & - & 78.8 & 56.5 & 49.4 \\
\hline 6240.645 & FeI & 2.22 & -3.189 & 94.1 & 91.8 & - & 116.5 & 101.9 & 90.0 \\
\hline 6271.283 & FeI & 3.33 & -2.544 & 59.5 & 58.3 & 56.1 & 76.2 & 61.2 & - \\
\hline 6297.792 & FeI & 2.22 & -2.646 & 123.6 & 122.6 & 117.6 & 137.5 & 130.5 & - \\
\hline 6315.813 & $\mathrm{FeI}$ & 4.07 & -1.319 & - & - & - & - & - & - \\
\hline 6322.691 & FeI & 2.59 & -2.227 & 123.3 & 124.2 & 119.9 & 138.0 & 125.8 & 113.6 \\
\hline 6380.750 & FeI & 4.19 & -1.129 & 86.5 & 90.9 & 89.7 & - & 103.1 & 74.2 \\
\hline 6385.726 & FeI & 4.73 & -1.672 & - & - & - & 44.4 & 28.9 & 20.0 \\
\hline 6392.538 & $\mathrm{FeI}$ & 2.28 & -3.804 & 66.4 & - & 61.5 & 81.7 & 56.3 & 50.3 \\
\hline 6498.945 & FeI & 0.96 & -4.507 & 113.4 & 104.6 & 103.7 & - & - & - \\
\hline 6608.044 & FeI & 2.28 & -3.878 & 56.1 & 49.6 & - & - & 54.3 & 46.6 \\
\hline 6627.560 & FeI & 4.55 & -1.319 & 48.7 & 49.9 & 49.7 & 65.6 & 51.4 & 47.6 \\
\hline 6633.427 & $\mathrm{FeI}$ & 4.83 & -1.034 & 46.6 & 51.8 & 51.8 & 66.0 & 71.3 & 37.7 \\
\hline 6634.123 & FeI & 4.79 & -0.645 & 66.1 & 71.9 & 74.2 & 85.5 & - & - \\
\hline 6646.966 & FeI & 2.61 & -3.743 & - & - & 39.0 & 64.0 & 60.4 & 32.1 \\
\hline 6653.911 & FeI & 4.15 & -2.228 & - & - & 29.7 & 45.0 & - & 20.2 \\
\hline 6696.322 & FeI & 4.83 & -1.350 & 30.2 & 29.4 & 32.7 & 44.5 & 33.4 & 24.3 \\
\hline 6699.136 & FeI & 4.59 & -1.892 & 20.9 & 20.8 & 22.8 & 32.8 & 32.3 & 16.9 \\
\hline 6703.576 & FeI & 2.76 & -2.991 & 79.1 & 77.9 & 75.0 & 93.3 & 85.4 & 69.1 \\
\hline 6704.500 & FeI & 4.22 & -2.426 & 17.7 & 18.6 & 17.4 & 26.3 & - & - \\
\hline 6705.105 & FeI & 4.61 & -0.908 & 68.7 & 70.9 & 72.4 & 83.6 & 86.1 & 61.8 \\
\hline 6710.323 & FeI & 1.48 & -4.650 & 64.9 & 54.3 & 56.7 & 89.0 & 89.9 & 53.4 \\
\hline 6713.745 & FeI & 4.79 & -1.300 & 33.0 & 35.2 & 40.5 & 51.6 & 50.0 & 31.5 \\
\hline 6725.364 & FeI & 4.10 & -2.055 & 35.7 & 38.2 & 37.1 & 51.8 & 49.3 & 33.1 \\
\hline 6726.673 & FeI & 4.61 & -1.004 & 64.6 & 66.6 & 68.3 & 78.6 & 68.1 & 61.3 \\
\hline 6732.068 & $\mathrm{FeI}$ & 4.58 & -2.015 & - & - & - & 30.3 & - & - \\
\hline 6733.153 & FeI & 4.64 & -1.302 & 43.9 & 43.8 & 46.3 & 59.3 & 60.8 & 40.9 \\
\hline 6739.524 & FeI & 1.56 & -4.819 & 54.3 & 42.5 & 44.1 & 77.3 & 54.8 & 43.0 \\
\hline 6745.113 & FeI & 4.58 & -1.888 & 23.0 & 21.8 & 24.9 & 39.6 & - & 16.4 \\
\hline 6746.975 & FeI & 2.61 & -4.157 & 24.3 & - & 24.0 & 42.1 & 23.2 & 16.5 \\
\hline 6750.164 & FeI & 2.42 & -2.493 & 126.5 & 124.4 & 121.9 & 142.5 & 131.0 & 113.8 \\
\hline 6752.716 & FeI & 4.64 & -1.011 & 63.4 & 64.3 & 66.8 & - & 75.5 & - \\
\hline 6786.860 & $\mathrm{FeI}$ & 4.19 & -1.710 & 46.3 & 46.1 & 58.8 & 69.3 & 58.9 & 44.8 \\
\hline 6806.856 & FeI & 2.73 & -2.923 & 77.0 & - & 83.1 & 105.0 & 92.0 & 75.1 \\
\hline 6810.267 & FeI & 4.61 & -0.826 & 68.8 & 72.2 & 85.7 & 94.0 & 75.4 & 69.4 \\
\hline 6820.374 & FeI & 4.64 & -1.024 & 64.0 & 66.6 & 66.4 & 81.7 & 67.9 & 61.0 \\
\hline 6828.596 & FeI & 4.64 & -0.648 & 75.0 & - & - & - & 89.7 & 75.5 \\
\hline 6837.013 & FeI & 4.59 & -1.555 & 31.1 & 35.1 & 35.2 & 44.7 & 37.1 & 29.8 \\
\hline 6839.835 & FeI & 2.56 & -3.185 & 75.6 & 71.0 & 72.7 & 92.8 & 94.6 & 67.4 \\
\hline 6842.689 & FeI & 4.64 & -1.018 & 59.2 & 63.9 & 61.2 & 72.4 & 71.6 & 56.2 \\
\hline 6843.655 & FeI & 4.55 & -0.705 & 80.8 & 86.2 & 84.5 & 91.4 & 95.2 & 76.9 \\
\hline 6855.166 & FeI & 4.56 & -0.568 & 91.3 & 98.8 & 96.3 & 106.2 & 104.1 & 86.8 \\
\hline 6855.723 & FeI & 4.61 & -1.393 & 44.5 & 49.7 & 47.3 & 58.7 & - & 37.2 \\
\hline 6857.251 & FeI & 4.07 & -1.985 & 43.4 & 48.3 & 44.1 & 58.6 & 50.4 & 37.9 \\
\hline 6858.155 & FeI & 4.61 & -0.878 & 70.0 & 78.7 & 77.2 & 82.9 & 95.0 & 68.1 \\
\hline 6861.945 & FeI & 2.42 & -3.613 & 60.4 & 58.7 & 59.3 & 82.9 & 80.3 & 51.4 \\
\hline 6862.496 & $\mathrm{FeI}$ & 4.56 & -1.294 & 48.2 & 51.8 & 55.3 & 65.2 & 68.4 & 41.8 \\
\hline 4656.981 & FeII & 2.89 & -3.544 & 56.9 & 75.0 & 74.5 & 56.1 & 78.2 & 60.1 \\
\hline 4993.358 & FeII & 2.81 & -3.694 & 61.0 & 74.8 & 74.4 & 69.3 & 63.9 & 59.1 \\
\hline 5197.576 & FeII & 3.23 & -2.219 & 106.2 & 133.1 & 119.9 & 103.5 & 126.2 & 106.7 \\
\hline
\end{tabular}


R. Smiljanic et al.: Abundance analysis of barium and mild barium stars, Online Material p 6

Table 8. continued.

\begin{tabular}{|c|c|c|c|c|c|c|c|c|c|}
\hline$\lambda(\AA)$ & Elem. & $x(\mathrm{eV})$ & $\log g f$ & HR 440 & HR 649 & HR 1016 & HR 1326 & HR 2392 & HR 4608 \\
\hline 5234.630 & FeII & 3.22 & -2.034 & 108.4 & 133.8 & 119.4 & 97.2 & 114.3 & 110.7 \\
\hline 5264.812 & FeII & 3.33 & -2.917 & 67.9 & 88.6 & 78.0 & 59.2 & 68.2 & 64.2 \\
\hline 5325.560 & FeII & 3.22 & -3.099 & 65.9 & 84.6 & 77.7 & 60.1 & 75.6 & 60.3 \\
\hline 5414.075 & FeII & 3.22 & -3.478 & 45.9 & 58.5 & 55.1 & 42.6 & 56.3 & 45.6 \\
\hline 5425.257 & FeII & 3.20 & -3.228 & 54.2 & 75.4 & 68.4 & 54.1 & 71.9 & 58.0 \\
\hline 6149.249 & FeII & 3.89 & -2.612 & 49.3 & 67.6 & 64.8 & 46.9 & 65.6 & 49.9 \\
\hline 6247.562 & FeII & 3.89 & -2.193 & 64.9 & 88.5 & 79.3 & 54.7 & 66.9 & 68.8 \\
\hline 6369.463 & FeII & 2.89 & -3.963 & 38.9 & 56.1 & 49.4 & 37.6 & - & 33.4 \\
\hline 6416.928 & FeII & 3.89 & -2.456 & 51.9 & 70.6 & 78.7 & 64.7 & 65.3 & 62.4 \\
\hline 6456.391 & FeII & 3.90 & -1.991 & 74.6 & 101.7 & 92.1 & 71.9 & 92.8 & 81.5 \\
\hline 4792.862 & CoI & 3.25 & - & 69.2 & 61.4 & 61.0 & 81.5 & 75.6 & 62.0 \\
\hline 4813.479 & CoI & 3.21 & - & 87.2 & 80.4 & 80.7 & 103.2 & - & 75.3 \\
\hline 5212.691 & $\mathrm{CoI}$ & 3.51 & - & 52.7 & 43.2 & 45.3 & 73.7 & 53.3 & 47.2 \\
\hline 5280.629 & $\mathrm{CoI}$ & 3.63 & - & 45.4 & 39.3 & 39.2 & 60.6 & 44.3 & 36.4 \\
\hline 5342.708 & $\mathrm{CoI}$ & 4.02 & - & 55.6 & 51.9 & 58.2 & 67.8 & 61.5 & 46.2 \\
\hline 5359.192 & $\mathrm{CoI}$ & 4.15 & - & 26.5 & 20.9 & 20.8 & 30.8 & 26.6 & 17.4 \\
\hline 5454.572 & CoI & 4.07 & - & 32.2 & 29.8 & 35.3 & 50.7 & 41.2 & 26.9 \\
\hline 5647.234 & CoI & 2.28 & - & 63.6 & 54.1 & 41.5 & 75.7 & 71.8 & 49.4 \\
\hline 6455.001 & CoI & 3.63 & - & - & - & 33.3 & 59.9 & - & - \\
\hline 6632.472 & $\mathrm{CoI}$ & 2.28 & - & 44.3 & 37.5 & 34.1 & 62.9 & 62.8 & 32.2 \\
\hline 4935.831 & NiI & 3.94 & -0.335 & 82.4 & 86.5 & 84.1 & 91.5 & - & - \\
\hline 4946.029 & NiI & 3.80 & -1.143 & 45.5 & 41.6 & 44.1 & 59.0 & 62.3 & 40.9 \\
\hline 4953.200 & NiI & 3.74 & -0.494 & 88.1 & 85.1 & 81.7 & 104.1 & - & - \\
\hline 5010.934 & $\mathrm{NiI}$ & 3.63 & -0.833 & 76.8 & 81.0 & 80.4 & 86.0 & 80.5 & 72.8 \\
\hline 5032.723 & $\mathrm{NiI}$ & 3.90 & -1.045 & 48.3 & 48.3 & 48.7 & 71.0 & 65.4 & 44.0 \\
\hline 5094.406 & $\mathrm{NiI}$ & 3.83 & -0.974 & 54.4 & 52.7 & 57.4 & 73.6 & 58.6 & 50.9 \\
\hline 5197.157 & NiI & 3.90 & -0.957 & 49.4 & 47.5 & 48.7 & 73.4 & 51.0 & 40.3 \\
\hline 5220.300 & $\mathrm{NiI}$ & 3.74 & -1.149 & 51.1 & 49.6 & 51.7 & 63.5 & 55.9 & 45.8 \\
\hline 5392.330 & $\mathrm{NiI}$ & 4.15 & -1.182 & 28.2 & 26.0 & 25.6 & 35.0 & 23.4 & 19.6 \\
\hline 5435.866 & NiI & 1.99 & -2.379 & 95.4 & 91.9 & 93.4 & 106.8 & 102.6 & 89.9 \\
\hline 5452.860 & NiI & 3.84 & -1.250 & - & 34.3 & 38.7 & 52.9 & 39.9 & 29.4 \\
\hline 5494.876 & NiI & 4.10 & -0.839 & 42.2 & 43.4 & 52.8 & 64.3 & 38.0 & 39.0 \\
\hline 5587.853 & NiI & 1.93 & -2.342 & 107.2 & 104.0 & 97.2 & 112.7 & - & - \\
\hline 5625.312 & NiI & 4.09 & -0.468 & 61.0 & 68.6 & 59.9 & - & 86.4 & 65.6 \\
\hline 5628.354 & NiI & 4.09 & -0.939 & - & 35.6 & - & - & 62.2 & 37.9 \\
\hline 5637.128 & NiI & 4.09 & -0.630 & 61.4 & 62.9 & 54.9 & 67.0 & 61.6 & 53.6 \\
\hline 5748.346 & $\mathrm{NiI}$ & 1.68 & -3.122 & 78.7 & 73.2 & 69.0 & 94.3 & - & 72.0 \\
\hline 5846.986 & $\mathrm{NiI}$ & 1.68 & -3.215 & 70.9 & 61.4 & 60.9 & 87.7 & 86.6 & 64.1 \\
\hline 6086.276 & $\mathrm{NiI}$ & 4.26 & -0.291 & 64.2 & 68.1 & 62.2 & 69.5 & 77.5 & - \\
\hline 6176.807 & $\mathrm{NiI}$ & 4.09 & -0.096 & 84.8 & 88.8 & 90.1 & 95.6 & 97.7 & 83.3 \\
\hline 6177.236 & $\mathrm{NiI}$ & 1.83 & -3.381 & 50.0 & 43.4 & 45.0 & 65.7 & 58.5 & 46.9 \\
\hline 6186.709 & $\mathrm{NiI}$ & 4.10 & -0.799 & 48.7 & 48.5 & 51.3 & 63.9 & 47.6 & 43.1 \\
\hline 6191.187 & $\mathrm{NiI}$ & 1.68 & -2.249 & 127.4 & 122.3 & 119.3 & 138.9 & 128.9 & 118.5 \\
\hline 6327.604 & $\mathrm{NiI}$ & 1.68 & -2.802 & 93.9 & 87.6 & 85.7 & 111.7 & 111.8 & 88.5 \\
\hline 6635.137 & $\mathrm{NiI}$ & 4.42 & -0.581 & 44.9 & 47.4 & 46.3 & 56.5 & 59.1 & 33.7 \\
\hline 6767.784 & $\mathrm{NiI}$ & 1.83 & -2.000 & 136.0 & 132.1 & 130.4 & - & 136.9 & 121.6 \\
\hline 6772.321 & $\mathrm{NiI}$ & 3.66 & -0.804 & 81.9 & 81.9 & 84.1 & 95.6 & 79.4 & 70.1 \\
\hline 6842.043 & NiI & 3.66 & -1.209 & 50.9 & 50.8 & 51.0 & 62.3 & 61.1 & 44.7 \\
\hline 5218.209 & $\mathrm{CuI}$ & 3.82 & - & 80.0 & 77.2 & 76.4 & 92.6 & 84.2 & 73.8 \\
\hline 5220.086 & $\mathrm{CuI}$ & 3.82 & - & 43.9 & 46.7 & 42.0 & 50.1 & - & 58.4 \\
\hline 5782.136 & $\mathrm{CuI}$ & 1.64 & - & 168.6 & 145.4 & - & 200.4 & 195.3 & 143.0 \\
\hline 4810.537 & $\mathrm{ZnI}$ & 4.08 & -0.433 & 87.5 & 101.6 & 96.3 & 81.0 & 91.8 & 84.3 \\
\hline 4607.338 & SrI & 0.00 & 0.069 & 85.5 & 94.4 & 91.8 & 108.1 & 157.9 & 106.9 \\
\hline 4883.690 & YII & 1.08 & 0.103 & 96.8 & 114.7 & 108.9 & 107.5 & 242.6 & 124.3 \\
\hline 4900.124 & YII & 1.03 & -0.131 & 93.7 & 118.1 & 96.7 & 100.8 & 222.2 & 119.2 \\
\hline 5087.426 & YII & 1.08 & -0.197 & 85.7 & 107.6 & 95.8 & 91.6 & 178.3 & 111.2 \\
\hline 5200.415 & YII & 0.99 & -0.570 & 77.2 & 97.5 & 84.2 & 89.4 & 138.9 & 98.9 \\
\hline 5289.820 & YII & 1.03 & -1.698 & 17.7 & 30.0 & 25.5 & 25.5 & 80.4 & 39.7 \\
\hline 5402.780 & YII & 1.84 & -0.434 & 23.9 & 50.1 & 43.8 & 45.4 & 91.4 & 55.6 \\
\hline 4739.454 & ZrI & 0.65 & 0.120 & 38.3 & 44.8 & 39.3 & 66.7 & 124.4 & 72.8 \\
\hline 4613.921 & ZrII & 0.97 & -0.528 & 67.7 & 75.8 & 77.6 & 84.3 & 113.4 & 76.3 \\
\hline 5112.279 & ZrII & 1.66 & -0.585 & 32.0 & 47.4 & 40.2 & 41.4 & 94.0 & 62.6 \\
\hline 5853.688 & BaII & 0.60 & -0.635 & 114.7 & 146.4 & 131.7 & 119.4 & 331.4 & 173.5 \\
\hline 6141.727 & BaII & 0.70 & 0.623 & 196.2 & 246.6 & 230.1 & 209.4 & 631.0 & 310.3 \\
\hline 6496.908 & BaII & 0.60 & 0.558 & 179.0 & 220.7 & 211.1 & 184.3 & 453.5 & 260.9 \\
\hline 4662.512 & LaII & 0.00 & -0.968 & 52.3 & 62.3 & 57.7 & 61.0 & 130.7 & 79.3 \\
\hline 4748.737 & LaII & 0.93 & -0.369 & 23.9 & - & 31.1 & 31.2 & 87.0 & 55.1 \\
\hline
\end{tabular}


R. Smiljanic et al.: Abundance analysis of barium and mild barium stars, Online Material $p 7$

Table 8. continued.

\begin{tabular}{cccccccccc}
\hline \hline$\lambda(\AA)$ & Elem. & $\chi(\mathrm{eV})$ & $\log g f$ & HR 440 & HR 649 & HR 1016 & HR 1326 & HR 2392 & HR 4608 \\
\hline 6320.429 & LaII & 0.17 & -0.984 & 32.9 & 46.2 & 37.9 & 43.9 & 147.5 & 69.4 \\
6390.493 & LaII & 0.32 & -1.115 & 35.9 & 52.1 & 39.1 & 50.9 & 122.2 & 62.2 \\
4523.080 & CeII & 0.52 & 0.470 & 72.4 & 88.3 & 80.0 & 86.8 & 186.0 & 103.7 \\
4562.367 & CeII & 0.48 & 0.420 & 65.7 & 84.5 & 75.8 & 74.4 & 137.3 & 93.1 \\
4628.160 & CeII & 0.52 & 0.410 & 67.3 & 87.3 & 81.9 & 75.1 & 189.8 & 105.0 \\
4773.959 & CeII & 0.92 & 0.214 & 34.6 & 42.7 & 40.1 & 53.6 & 90.0 & 54.8 \\
5274.236 & CeII & 1.04 & 0.413 & 40.0 & 51.6 & 46.6 & 48.1 & 98.0 & 65.6 \\
5089.831 & NdII & 0.20 & -1.023 & 25.0 & 31.7 & 31.3 & 46.5 & 80.9 & 54.1 \\
5319.820 & NIdI & 0.55 & -0.129 & 58.6 & 72.8 & 63.9 & 64.7 & 108.7 & 82.6 \\
4566.233 & SmII & 0.33 & -0.170 & 40.7 & 47.5 & 45.3 & 54.9 & 91.1 & 55.3 \\
6645.127 & EuII & 1.38 & 0.444 & - & - & 31.5 & 35.0 & 60.0 & 35.2 \\
5419.880 & GdII & 1.31 & 0.088 & 7.0 & - & 8.5 & 13.2 & 17.0 & - \\
\hline
\end{tabular}

Table 9. Equivalent widths for the stars $\epsilon$ Vir, HR 5058, HR 5802, HR 7321, HR 8115, HR 8204, HD 205011 and HR 8878.

\begin{tabular}{|c|c|c|c|c|c|c|c|c|c|}
\hline$\lambda(\AA)$ & Elem. & $\epsilon$ Vir & HR 5058 & HR 5802 & HR 7321 & HR 8115 & HR 8204 & HD 205011 & HR 8878 \\
\hline 6154.230 & $\mathrm{NaI}$ & 94.4 & 87.1 & 81.0 & 70.7 & 95.1 & 67.7 & 80.7 & 63.5 \\
\hline 6160.753 & $\mathrm{NaI}$ & 113.1 & 122.6 & 102.3 & 92.7 & 114.7 & 93.4 & 101.8 & 87.1 \\
\hline 4571.102 & $\mathrm{MgI}$ & 204.6 & 244.0 & 201.8 & 213.8 & 208.9 & 214.8 & 225.0 & - \\
\hline 4730.038 & $\mathrm{MgI}$ & 122.9 & 143.3 & 125.9 & 119.2 & 120.5 & 133.0 & - & 112.4 \\
\hline 5711.095 & $\mathrm{MgI}$ & 145.0 & 140.4 & 139.9 & 134.2 & 140.9 & 132.2 & 136.4 & 145.0 \\
\hline 5785.285 & $\mathrm{MgI}$ & 88.2 & 92.8 & 81.9 & 81.3 & 86.9 & 75.3 & 84.0 & 71.4 \\
\hline 6696.032 & AlI & 74.7 & 77.0 & 71.7 & 47.3 & 72.1 & - & 74.3 & 80.9 \\
\hline 6698.669 & AlI & 54.5 & 70.6 & 54.0 & 66.5 & 55.6 & 69.0 & 59.4 & 55.2 \\
\hline 5517.533 & SiI & 36.9 & 36.7 & 29.3 & 37.2 & - & 41.3 & - & 22.4 \\
\hline 5665.563 & SiI & 88.5 & - & - & 75.9 & 85.0 & - & 84.5 & 64.5 \\
\hline 5684.484 & SiI & 97.3 & 85.0 & 85.6 & 82.2 & 89.1 & 98.3 & - & 69.7 \\
\hline 5690.433 & SiI & 88.8 & 84.2 & 78.2 & 75.5 & 79.8 & 89.1 & - & 64.0 \\
\hline 5701.108 & SiI & 73.5 & 60.5 & 66.2 & 59.0 & 65.6 & 64.0 & - & 52.7 \\
\hline 5708.405 & SiI & 117.9 & - & - & 115.4 & 120.5 & - & 120.4 & - \\
\hline 5753.622 & SiI & 88.5 & - & - & 77.1 & 88.4 & - & 83.6 & 57.3 \\
\hline 5772.149 & SiI & 96.5 & 101.5 & - & 77.0 & 91.5 & - & 86.3 & - \\
\hline 5793.080 & SiI & 76.0 & 90.1 & 66.4 & 65.7 & 77.0 & 82.3 & 70.1 & 52.2 \\
\hline 6131.577 & SiI & 47.9 & 42.2 & 41.7 & 38.4 & 48.5 & 44.6 & 40.1 & 27.6 \\
\hline 6131.858 & SiI & 46.8 & - & 43.1 & 41.9 & 50.0 & 65.5 & 47.5 & - \\
\hline 6142.494 & SiI & 59.8 & 61.9 & 53.7 & 49.1 & 58.7 & 78.2 & 52.4 & 32.7 \\
\hline 6145.020 & SiI & 62.5 & 53.2 & 55.1 & 50.2 & 60.7 & 61.4 & 50.6 & 37.5 \\
\hline 6243.823 & SiI & 75.7 & 69.6 & 73.8 & 66.2 & 82.3 & 101.1 & 77.6 & 50.5 \\
\hline 6721.844 & SiI & 75.0 & - & 70.8 & 63.7 & 80.2 & 74.5 & 77.0 & 45.1 \\
\hline 5261.708 & $\mathrm{CaI}$ & 146.5 & 146.5 & 136.7 & 141.3 & 150.7 & - & 141.0 & 139.9 \\
\hline 5581.979 & $\mathrm{CaI}$ & 138.9 & - & 135.1 & 138.0 & 142.7 & - & - & 138.5 \\
\hline 5590.126 & $\mathrm{CaI}$ & 128.3 & 124.9 & 119.6 & 121.3 & 126.0 & 133.7 & 120.3 & 128.0 \\
\hline 5867.572 & $\mathrm{CaI}$ & 56.4 & 60.1 & 50.1 & 49.9 & 53.3 & 37.1 & 50.8 & 55.9 \\
\hline 6161.295 & $\mathrm{CaI}$ & 114.3 & 100.9 & 108.1 & 108.3 & 117.5 & - & 116.5 & 116.6 \\
\hline 6163.754 & $\mathrm{CaI}$ & 99.2 & - & - & 88.6 & 98.5 & - & 90.2 & 96.0 \\
\hline 6166.440 & $\mathrm{CaI}$ & 110.3 & 124.5 & 105.7 & 104.3 & 110.4 & 115.8 & 105.9 & - \\
\hline 6169.044 & $\mathrm{CaI}$ & 138.0 & - & 130.0 & 127.4 & 138.0 & 133.3 & 136.1 & 136.0 \\
\hline 6449.820 & $\mathrm{CaI}$ & 143.1 & 142.7 & 134.4 & 133.5 & 137.4 & 144.8 & 134.7 & 138.0 \\
\hline 6455.605 & $\mathrm{CaI}$ & 107.6 & 126.5 & 100.7 & 95.3 & 106.1 & 91.0 & 101.2 & 110.5 \\
\hline 6499.654 & $\mathrm{CaI}$ & 134.9 & - & 124.6 & 128.4 & 137.7 & 133.9 & 132.4 & 134.1 \\
\hline 6798.467 & $\mathrm{CaI}$ & 37.6 & 47.0 & - & 29.2 & - & - & 34.9 & 33.8 \\
\hline 5318.346 & ScII & 48.9 & 41.8 & 43.3 & 39.2 & 48.8 & 74.9 & 40.7 & 44.2 \\
\hline 5357.190 & ScII & 21.4 & 21.6 & 19.5 & 17.6 & 21.3 & 28.4 & 19.2 & 23.7 \\
\hline 5526.815 & ScII & 123.5 & 130.8 & 111.8 & 121.8 & 134.7 & - & 128.9 & 114.3 \\
\hline 5657.874 & ScII & 129.0 & 122.8 & 116.6 & 118.2 & 123.5 & - & 116.2 & 118.6 \\
\hline 5684.189 & ScII & 91.3 & 87.3 & 82.7 & 82.8 & 87.8 & 119.7 & 83.6 & 89.3 \\
\hline 6245.660 & ScII & 91.4 & 98.4 & 88.0 & 83.3 & 94.4 & 129.5 & 92.2 & 91.0 \\
\hline 6320.867 & ScII & 51.6 & 47.6 & 39.3 & 36.8 & 42.0 & 65.0 & 39.9 & 47.5 \\
\hline 4518.023 & TiI & 125.6 & 148.4 & 120.9 & 127.2 & 127.8 & 145.9 & 131.5 & - \\
\hline 4548.765 & TiI & 126.5 & - & 122.3 & 124.7 & 130.9 & 147.7 & 131.4 & - \\
\hline 4555.485 & TiI & 120.9 & - & 118.1 & 123.8 & 128.9 & - & - & - \\
\hline 4562.625 & TiI & 61.7 & 91.7 & 60.6 & 65.7 & 65.8 & 42.1 & 70.4 & 108.8 \\
\hline 4617.254 & TiI & 105.9 & 113.9 & - & 106.0 & 108.6 & 106.9 & 107.2 & 126.7 \\
\hline 4758.120 & TiI & 85.1 & 101.1 & 82.9 & 85.1 & 85.4 & 98.8 & 87.7 & 104.1 \\
\hline 4759.272 & TiI & 86.2 & 111.2 & 84.1 & 87.6 & 87.4 & 96.2 & 90.3 & 107.2 \\
\hline
\end{tabular}


R. Smiljanic et al.: Abundance analysis of barium and mild barium stars, Online Material p 8

Table 9. continued.

\begin{tabular}{|c|c|c|c|c|c|c|c|c|c|}
\hline$\lambda(\AA)$ & Elem. & $\epsilon \mathrm{Vir}$ & HR 5058 & HR 5802 & HR 7321 & HR 8115 & HR 8204 & HD 205011 & HR 8878 \\
\hline 4778.259 & TiI & 49.8 & 60.6 & 45.8 & 54.2 & 53.8 & 43.4 & 57.7 & 77.5 \\
\hline 4926.147 & TiI & 37.5 & 58.6 & 45.2 & 42.3 & 36.8 & - & 43.7 & 80.3 \\
\hline 5022.871 & TiI & 131.5 & 148.4 & 128.5 & 135.4 & 138.2 & 141.3 & 137.2 & - \\
\hline 5024.842 & TiI & 125.0 & 132.2 & 122.3 & 125.7 & 129.7 & 122.2 & 126.0 & - \\
\hline 5071.472 & TiI & 89.2 & 105.5 & 86.1 & - & 85.1 & 68.6 & 83.6 & 123.4 \\
\hline 5113.448 & TiI & 78.1 & 86.4 & 75.5 & 76.6 & 75.1 & 46.0 & 75.2 & 118.7 \\
\hline 5145.464 & TiI & 87.7 & 108.3 & 86.4 & 88.2 & 88.0 & 81.2 & 92.0 & 125.0 \\
\hline 5147.479 & TiI & 107.3 & - & 109.0 & 116.7 & 117.0 & - & - & - \\
\hline 5152.185 & TiI & 93.1 & 121.8 & - & 96.8 & 95.6 & 92.2 & 99.5 & 145.2 \\
\hline 5211.206 & TiI & 38.2 & 44.1 & 30.7 & - & 40.2 & - & 36.4 & - \\
\hline 5219.700 & TiI & 94.2 & 129.0 & 86.8 & 101.2 & 102.2 & 79.7 & 101.8 & 146.1 \\
\hline 5295.780 & TiI & 55.4 & 69.9 & 56.7 & 56.5 & 54.9 & 30.5 & 59.0 & 93.1 \\
\hline 5426.236 & TiI & 49.9 & 85.6 & 45.1 & 55.0 & 47.2 & 22.0 & 55.5 & 112.7 \\
\hline 5471.197 & TiI & 47.0 & 63.4 & 38.2 & 50.7 & 57.7 & 40.4 & 57.3 & 76.5 \\
\hline 5490.150 & TiI & 78.5 & 93.6 & 72.8 & 79.1 & 84.7 & 70.0 & 83.6 & 105.8 \\
\hline 5648.567 & TiI & 45.3 & 64.3 & 42.6 & 42.3 & 39.3 & 22.8 & 42.6 & 66.0 \\
\hline 5739.464 & TiI & 32.4 & 41.4 & 28.0 & 34.9 & 32.0 & 18.5 & 33.4 & 54.7 \\
\hline 5866.452 & TiI & 113.3 & 141.5 & 104.2 & 110.3 & 113.3 & 87.4 & 114.4 & 148.6 \\
\hline 6064.629 & TiI & 54.9 & 87.5 & 58.1 & 59.7 & 52.4 & 38.5 & 57.7 & 96.5 \\
\hline 6126.224 & TiI & 79.2 & - & 81.0 & 87.8 & 88.1 & - & - & 119.2 \\
\hline 6258.104 & TiI & 112.7 & 125.3 & 110.4 & - & - & 89.0 & 106.5 & 147.4 \\
\hline 6861.500 & TiI & 36.5 & 43.0 & 29.7 & 34.0 & 35.7 & - & 40.4 & 53.3 \\
\hline 4524.691 & TiII & 71.5 & - & - & 74.5 & - & 118.0 & - & 78.1 \\
\hline 4568.345 & TiII & 81.9 & 86.0 & 78.7 & 78.6 & 87.6 & 116.3 & 83.3 & 91.2 \\
\hline 4583.415 & TiII & 82.4 & 78.0 & 76.7 & 78.6 & 88.5 & 130.1 & 81.0 & 82.2 \\
\hline 4657.209 & TiII & 108.7 & 109.8 & 101.3 & 102.1 & 109.8 & - & 107.1 & 112.9 \\
\hline 4798.539 & TilI & 87.1 & 86.5 & 80.1 & 87.3 & 90.9 & 118.3 & 86.4 & 96.1 \\
\hline 5211.544 & TilI & 69.9 & 59.0 & 58.8 & 63.7 & 74.5 & 108.8 & 62.2 & 63.8 \\
\hline 5336.783 & TilI & 116.9 & 113.5 & 106.2 & 114.5 & 123.3 & - & 118.0 & 115.2 \\
\hline 5381.020 & TiII & 122.8 & - & 112.4 & - & 128.0 & - & - & - \\
\hline 5418.756 & TiII & 92.7 & 94.3 & 81.1 & 88.5 & 95.8 & 145.2 & 88.3 & 90.4 \\
\hline 5657.436 & VI & 46.1 & 73.8 & 46.8 & 46.8 & 41.7 & - & 50.8 & 79.4 \\
\hline 5668.362 & VI & 43.5 & 63.0 & 46.5 & 42.8 & 39.3 & 18.8 & 45.8 & 80.0 \\
\hline 5670.851 & VI & 86.8 & 107.5 & 84.8 & 86.2 & 80.6 & 42.2 & 89.8 & 129.9 \\
\hline 5727.661 & VI & 55.1 & - & - & 53.8 & 51.4 & - & 54.2 & 100.6 \\
\hline 6090.216 & VI & 100.2 & 116.4 & 99.5 & 96.9 & 92.9 & 66.9 & 97.1 & 121.6 \\
\hline 6135.370 & VI & 58.7 & 90.3 & 55.5 & 59.1 & 61.0 & 38.8 & 63.6 & 97.7 \\
\hline 6150.154 & VI & 66.8 & 106.7 & 64.0 & 71.5 & 70.2 & 47.0 & 76.9 & 126.7 \\
\hline 6199.186 & VI & 76.5 & 120.8 & 71.5 & 81.7 & 78.7 & 38.5 & 89.2 & 144.5 \\
\hline 6216.358 & VI & 104.9 & 129.5 & 101.9 & 108.8 & 106.4 & 72.9 & 114.6 & 158.3 \\
\hline 6274.658 & VI & 58.4 & 98.7 & 59.2 & 60.7 & 57.8 & 33.3 & 67.6 & 114.5 \\
\hline 6285.165 & VI & 63.8 & 90.7 & 61.3 & 69.2 & 68.9 & 33.0 & 75.7 & 116.7 \\
\hline 4616.120 & $\mathrm{CrI}$ & 141.8 & - & 147.8 & 144.0 & 149.6 & - & - & - \\
\hline 4626.174 & $\mathrm{CrI}$ & 133.5 & 142.9 & 134.2 & 133.7 & 139.2 & - & 138.7 & 144.7 \\
\hline 4708.019 & $\mathrm{CrI}$ & 103.7 & - & - & 105.9 & 108.9 & - & 120.2 & 94.7 \\
\hline 4737.355 & $\mathrm{CrI}$ & 112.2 & 143.8 & 117.6 & 115.6 & 119.3 & - & 124.7 & 98.2 \\
\hline 4756.137 & $\mathrm{CrI}$ & 106.0 & 123.3 & 97.5 & 98.5 & 101.8 & - & 104.1 & 101.1 \\
\hline 4801.047 & $\mathrm{CrI}$ & 90.7 & - & 87.5 & 94.7 & 99.4 & - & 106.0 & 87.0 \\
\hline 4936.335 & $\mathrm{CrI}$ & 89.5 & 114.3 & 92.2 & 85.2 & 86.6 & 96.5 & 89.7 & 86.5 \\
\hline 4964.916 & $\mathrm{CrI}$ & 88.8 & 88.1 & - & 85.3 & 85.6 & 65.6 & 82.4 & 105.0 \\
\hline 5200.207 & $\mathrm{CrI}$ & 64.2 & 101.5 & 62.1 & 67.3 & 71.2 & - & 75.9 & 56.3 \\
\hline 5214.144 & $\mathrm{CrI}$ & 47.8 & 39.1 & 36.3 & 40.2 & 45.0 & 28.1 & 38.7 & 38.1 \\
\hline 5238.964 & $\mathrm{CrI}$ & 58.1 & 49.4 & 52.8 & 49.6 & 51.8 & 36.3 & 47.3 & 48.4 \\
\hline 5247.566 & $\mathrm{CrI}$ & 138.7 & 144.6 & 130.3 & 139.0 & 141.0 & 135.1 & 135.6 & - \\
\hline 5272.007 & $\mathrm{CrI}$ & 62.5 & 78.0 & 56.4 & 56.8 & 60.2 & 61.4 & 59.8 & 50.1 \\
\hline 5287.183 & $\mathrm{CrI}$ & 36.1 & 59.9 & 37.0 & 32.8 & 36.3 & - & 37.4 & 26.1 \\
\hline 5300.751 & $\mathrm{CrI}$ & 117.5 & 123.6 & 110.0 & 110.9 & 113.7 & 98.6 & 110.5 & 128.4 \\
\hline 5304.183 & $\mathrm{CrI}$ & 42.9 & 38.9 & 40.8 & 34.2 & 38.1 & 33.6 & 35.2 & 33.3 \\
\hline 5318.810 & $\mathrm{CrI}$ & 45.5 & - & 43.6 & 33.8 & 43.0 & 39.2 & 44.1 & 35.7 \\
\hline 5628.621 & $\mathrm{CrI}$ & 51.0 & 46.1 & 44.8 & - & 38.6 & 23.6 & - & 35.0 \\
\hline 5784.976 & $\mathrm{CrI}$ & 71.2 & 82.3 & 63.2 & 65.7 & 71.2 & 45.5 & 68.0 & 61.5 \\
\hline 5787.965 & $\mathrm{CrI}$ & 84.1 & 96.5 & 76.0 & 80.0 & 83.7 & 81.1 & 81.8 & 77.0 \\
\hline 6330.097 & $\mathrm{CrI}$ & 88.0 & 114.1 & 81.3 & 83.2 & 86.9 & 69.0 & 89.5 & 106.5 \\
\hline 4588.203 & CrII & 106.1 & 125.3 & 101.2 & 100.1 & 115.9 & - & 111.4 & 67.6 \\
\hline 4592.049 & CrII & 82.7 & 92.0 & 79.6 & 74.5 & 85.5 & 122.1 & 80.5 & 49.6 \\
\hline 5305.855 & CrII & 57.9 & - & 51.3 & 43.6 & 56.3 & 86.5 & 47.9 & 25.0 \\
\hline 5308.377 & CrII & 53.9 & 49.3 & 49.7 & 43.5 & 53.5 & 70.0 & 45.9 & - \\
\hline
\end{tabular}


R. Smiljanic et al.: Abundance analysis of barium and mild barium stars, Online Material p 9

Table 9. continued.

\begin{tabular}{|c|c|c|c|c|c|c|c|c|c|}
\hline$\lambda(\AA)$ & Elem. & $\epsilon$ Vir & HR 5058 & HR 5802 & HR 7321 & HR 8115 & HR 8204 & HD 205011 & HR 8878 \\
\hline 5313.526 & CrII & 67.9 & - & 59.7 & 53.8 & 67.5 & 126.5 & 61.9 & 36.2 \\
\hline 5502.025 & CrII & 51.0 & 66.3 & 42.7 & 48.6 & 61.6 & 89.4 & 60.9 & - \\
\hline 4739.113 & MnI & 107.9 & 117.3 & 106.1 & 102.2 & 106.6 & 117.9 & 106.3 & 95.5 \\
\hline 5413.684 & MnI & 66.7 & 70.2 & 53.1 & 53.5 & 60.3 & 55.4 & 61.1 & 35.3 \\
\hline 5420.350 & $\mathrm{MnI}$ & 192.6 & 199.9 & 172.5 & 182.7 & 184.4 & 121.0 & 181.7 & 189.4 \\
\hline 5432.548 & $\mathrm{MnI}$ & 164.5 & 179.9 & - & - & 160.4 & 107.8 & 167.1 & 192.9 \\
\hline 5537.765 & MnI & 117.6 & - & 104.2 & - & - & 113.3 & 128.4 & - \\
\hline 6013.497 & $\mathrm{MnI}$ & 147.7 & - & 132.2 & 135.2 & 141.5 & 125.9 & 138.9 & 136.2 \\
\hline 6021.803 & MnI & 149.1 & - & 133.0 & 138.9 & 143.3 & 128.3 & 140.3 & 128.6 \\
\hline 4523.400 & FeI & 83.3 & - & 77.1 & 78.4 & 84.6 & - & 87.3 & 75.1 \\
\hline 4537.676 & FeI & 50.9 & 50.7 & 44.3 & 43.9 & 48.5 & - & 47.9 & 49.2 \\
\hline 4556.925 & FeI & 68.5 & - & 64.3 & 64.1 & 70.3 & - & 76.0 & 56.8 \\
\hline 4585.343 & $\mathrm{FeI}$ & 49.9 & 44.8 & 48.1 & 43.4 & 51.2 & 45.3 & 50.0 & 28.0 \\
\hline 4593.555 & FeI & 69.1 & - & - & - & - & - & - & - \\
\hline 4598.125 & $\mathrm{FeI}$ & 123.5 & - & 114.4 & 117.7 & 120.3 & - & 114.7 & 110.4 \\
\hline 4602.000 & $\mathrm{FeI}$ & 127.9 & 144.8 & - & 129.1 & 134.9 & - & 134.1 & 131.9 \\
\hline 4741.535 & FeI & 117.0 & - & - & - & 122.1 & - & - & - \\
\hline 4749.961 & FeI & 68.7 & - & 66.3 & 64.3 & 67.0 & - & 68.3 & 53.2 \\
\hline 4793.961 & FeI & 31.3 & 44.0 & 33.7 & 33.9 & 33.1 & 27.3 & 36.8 & - \\
\hline 4794.355 & $\mathrm{FeI}$ & 45.8 & 51.8 & 46.6 & 47.1 & 47.1 & 32.7 & 48.4 & - \\
\hline 4798.273 & $\mathrm{FeI}$ & 76.2 & 71.1 & 69.3 & 73.5 & 77.0 & 74.1 & 71.8 & 60.4 \\
\hline 4798.743 & $\mathrm{FeI}$ & 84.0 & 91.5 & 77.3 & 86.2 & 88.4 & 78.6 & 86.6 & - \\
\hline 4808.147 & $\mathrm{FeI}$ & 68.4 & 59.6 & 60.7 & 64.9 & 67.3 & 48.9 & 63.2 & 60.5 \\
\hline 4907.733 & FeI & 108.8 & 104.9 & 102.6 & 102.4 & 106.0 & 100.3 & 102.2 & 92.9 \\
\hline 4908.032 & FeI & 85.7 & 81.7 & 80.9 & 75.2 & 81.1 & 68.6 & 78.5 & - \\
\hline 4911.788 & FeI & 77.3 & 66.1 & - & 71.0 & 75.4 & 65.4 & 69.1 & 62.8 \\
\hline 4961.915 & FeI & 72.4 & 88.6 & 67.7 & 64.4 & 70.0 & - & 69.2 & 62.8 \\
\hline 4962.565 & FeI & 90.8 & 81.1 & 83.4 & 79.8 & 84.7 & 90.1 & 78.4 & 75.7 \\
\hline 4969.916 & FeI & 115.3 & 115.6 & 104.8 & 104.6 & 110.2 & - & 104.7 & 101.2 \\
\hline 5023.189 & FeI & 78.2 & 74.5 & 71.8 & 71.3 & 79.0 & 70.1 & 75.5 & - \\
\hline 5025.091 & FeI & 49.7 & - & 45.3 & 44.2 & 48.3 & 42.5 & 46.8 & 34.7 \\
\hline 5025.313 & FeI & 47.6 & 63.5 & 45.1 & 44.9 & 51.2 & - & 48.6 & 28.4 \\
\hline 5054.647 & FeI & 83.5 & - & 80.3 & 76.1 & 81.5 & 88.6 & 79.3 & - \\
\hline 5067.162 & FeI & 116.3 & 122.4 & 107.4 & 104.0 & 109.4 & 123.8 & 105.4 & - \\
\hline 5072.677 & FeI & 104.9 & 113.4 & 99.9 & 98.2 & 102.0 & 113.4 & 97.7 & - \\
\hline 5109.649 & FeI & 116.8 & 118.1 & 108.7 & 108.8 & 120.8 & - & - & - \\
\hline 5127.680 & FeI & 89.6 & - & 84.9 & 96.4 & 97.0 & - & 102.7 & - \\
\hline 5196.065 & FeI & 107.5 & 110.9 & 100.7 & 101.2 & 105.0 & 116.2 & 103.6 & - \\
\hline 5197.929 & FeI & 72.4 & 74.8 & 62.9 & 64.2 & 70.8 & 73.9 & 65.9 & 50.3 \\
\hline 5213.818 & FeI & 31.9 & 30.3 & 22.8 & 26.6 & 32.7 & 19.0 & 26.3 & 26.0 \\
\hline 5223.188 & $\mathrm{FeI}$ & 68.9 & 63.1 & 57.9 & 63.1 & 74.1 & 61.4 & 64.4 & 56.1 \\
\hline 5242.491 & FeI & 133.7 & - & 121.5 & 120.6 & 127.9 & 139.4 & 119.4 & 111.6 \\
\hline 5243.773 & FeI & 102.2 & 97.2 & 88.5 & 93.3 & 100.3 & 100.6 & 90.6 & 83.1 \\
\hline 5247.049 & $\mathrm{FeI}$ & 142.3 & - & 132.1 & 145.4 & 149.5 & 146.6 & 146.3 & - \\
\hline 5320.040 & FeI & 51.8 & 52.4 & 48.8 & 48.2 & 55.2 & 40.8 & 50.3 & 45.1 \\
\hline 5321.109 & FeI & 75.0 & - & 72.0 & 71.4 & 78.0 & - & 77.2 & 60.9 \\
\hline 5379.574 & FeI & 102.2 & 117.8 & 93.0 & 95.3 & 104.1 & 107.1 & 100.3 & 85.6 \\
\hline 5389.486 & $\mathrm{FeI}$ & 113.0 & 111.4 & 106.0 & 105.7 & 112.1 & 125.4 & 105.9 & - \\
\hline 5395.222 & FeI & 49.7 & 53.6 & 43.5 & 39.6 & 48.6 & - & 47.0 & 35.1 \\
\hline 5412.791 & FeI & 52.7 & 48.4 & 42.1 & 43.1 & 47.7 & 45.2 & 43.4 & 37.9 \\
\hline 5432.946 & $\mathrm{FeI}$ & 107.6 & - & 92.7 & 93.9 & 105.3 & - & 95.9 & 80.0 \\
\hline 5436.297 & FeI & 80.7 & 67.8 & 65.6 & 66.4 & 73.5 & 63.3 & 65.8 & 51.6 \\
\hline 5473.168 & FeI & 54.9 & - & 47.2 & 53.8 & 63.0 & 96.6 & 62.2 & 34.1 \\
\hline 5483.108 & FeI & 85.5 & 84.9 & 74.9 & 81.4 & - & - & 84.5 & 64.9 \\
\hline 5491.845 & $\mathrm{FeI}$ & 49.3 & 57.7 & 43.4 & 44.1 & 54.6 & - & - & 26.8 \\
\hline 5494.474 & FeI & 74.7 & 67.1 & 66.3 & 67.7 & 76.7 & 78.1 & 72.6 & 53.2 \\
\hline 5508.419 & $\mathrm{FeI}$ & 41.9 & - & 35.5 & - & 44.5 & - & - & 21.8 \\
\hline 5522.454 & FeI & 78.3 & 81.7 & 69.6 & 75.9 & - & - & 82.0 & 61.4 \\
\hline 5560.207 & FeI & 87.9 & 76.6 & 75.7 & 76.6 & 80.8 & 79.7 & 74.8 & 63.4 \\
\hline 5577.013 & FeI & 35.1 & 31.3 & 24.8 & 24.0 & 30.8 & - & 26.5 & 14.1 \\
\hline 5587.573 & $\mathrm{FeI}$ & 76.7 & 76.8 & 67.8 & 69.2 & 74.2 & 57.7 & 70.1 & 55.1 \\
\hline 5635.824 & $\mathrm{FeI}$ & 79.2 & 68.1 & 70.0 & 65.5 & - & 53.0 & 64.5 & 57.5 \\
\hline 5636.705 & FeI & 67.1 & 67.3 & 54.6 & 54.3 & - & 42.9 & 55.4 & 49.2 \\
\hline 5638.262 & $\mathrm{FeI}$ & 122.9 & 128.8 & 111.6 & 114.2 & 116.1 & 126.5 & 112.0 & 102.7 \\
\hline 5641.436 & FeI & 120.0 & 116.4 & 109.5 & 107.7 & 110.1 & - & 107.3 & 97.7 \\
\hline 5646.697 & FeI & 39.0 & 46.8 & 31.3 & 30.5 & - & - & 31.3 & - \\
\hline 5650.019 & FeI & 75.6 & 87.0 & 67.5 & 64.9 & 67.5 & 55.7 & 68.0 & 54.6 \\
\hline
\end{tabular}


R. Smiljanic et al.: Abundance analysis of barium and mild barium stars, Online Material p 10

Table 9. continued.

\begin{tabular}{|c|c|c|c|c|c|c|c|c|c|}
\hline$\lambda(\AA)$ & Elem. & $\epsilon \mathrm{Vir}$ & HR 5058 & HR 5802 & HR 7321 & HR 8115 & HR 8204 & HD 205011 & HR 8878 \\
\hline 5652.319 & FeI & 69.2 & 61.5 & 61.5 & 58.0 & 60.1 & 43.3 & 58.6 & 50.1 \\
\hline 5661.348 & FeI & 68.8 & - & 63.1 & 59.0 & 64.7 & 53.7 & 64.6 & 50.0 \\
\hline 5680.240 & $\mathrm{FeI}$ & 48.5 & - & 44.7 & 40.8 & 43.5 & - & 46.7 & 35.7 \\
\hline 5701.557 & $\mathrm{FeI}$ & 148.1 & - & 135.6 & 135.3 & 143.6 & 144.3 & 140.5 & 141.5 \\
\hline 5705.473 & $\mathrm{FeI}$ & 79.5 & 67.7 & 72.6 & 65.9 & 73.9 & 60.5 & 68.4 & 61.4 \\
\hline 5731.761 & $\mathrm{FeI}$ & 96.5 & 105.5 & 85.9 & - & 96.9 & 101.2 & 90.8 & 78.4 \\
\hline 5738.240 & $\mathrm{FeI}$ & 41.6 & 36.1 & 32.0 & 36.3 & 36.4 & 24.0 & 33.5 & 21.7 \\
\hline 5775.069 & $\mathrm{FeI}$ & 99.8 & 93.8 & 96.5 & 87.0 & 96.6 & 92.3 & 90.9 & 79.8 \\
\hline 5778.463 & $\mathrm{FeI}$ & 74.4 & 76.2 & 72.6 & 65.2 & 70.2 & 50.5 & 69.5 & 64.9 \\
\hline 5784.666 & $\mathrm{FeI}$ & 66.7 & 73.3 & 60.7 & 65.2 & 67.2 & 50.8 & 65.3 & 60.1 \\
\hline 5811.916 & FeI & 34.7 & 35.7 & 29.2 & 30.0 & 33.1 & - & 30.9 & 24.8 \\
\hline 5814.805 & $\mathrm{FeI}$ & 56.8 & 60.0 & 48.1 & 51.0 & 53.1 & 48.0 & 51.8 & 42.4 \\
\hline 5835.098 & $\mathrm{FeI}$ & 47.1 & - & 37.6 & 37.1 & 42.9 & 33.1 & 41.0 & 29.7 \\
\hline 5849.681 & $\mathrm{FeI}$ & 33.7 & 37.7 & 27.9 & 25.2 & 27.3 & 14.1 & 25.9 & 25.3 \\
\hline 5852.222 & $\mathrm{FeI}$ & 84.3 & 83.0 & 72.1 & 69.7 & 75.4 & - & 72.1 & - \\
\hline 5855.086 & FeI & 56.1 & 55.5 & 44.9 & 42.1 & 46.7 & 35.6 & 44.1 & 35.1 \\
\hline 5856.096 & $\mathrm{FeI}$ & 75.5 & 83.9 & 62.7 & 62.0 & 69.8 & 60.4 & 63.7 & 53.1 \\
\hline 5859.596 & $\mathrm{FeI}$ & 108.6 & 115.8 & 97.1 & 96.7 & 107.4 & 112.9 & 101.0 & 82.8 \\
\hline 6005.551 & $\mathrm{FeI}$ & 79.1 & 94.7 & 66.4 & - & 73.9 & 50.0 & 74.9 & 67.9 \\
\hline 6007.968 & $\mathrm{FeI}$ & 99.5 & 104.5 & 84.9 & 85.6 & 94.5 & 94.5 & 90.9 & 70.9 \\
\hline 6012.212 & FeI & 79.0 & 75.1 & - & - & - & 45.5 & - & - \\
\hline 6078.499 & $\mathrm{FeI}$ & 117.1 & 106.7 & 113.3 & 100.9 & 105.4 & 104.7 & 99.1 & 86.9 \\
\hline 6079.014 & $\mathrm{FeI}$ & 85.7 & 76.6 & 81.3 & 70.6 & - & 62.4 & 67.5 & 61.2 \\
\hline 6093.666 & $\mathrm{FeI}$ & 68.6 & - & 59.3 & 58.4 & 63.4 & 49.7 & 60.3 & 41.9 \\
\hline 6098.250 & $\mathrm{FeI}$ & 51.9 & 77.2 & 46.7 & 47.4 & 50.4 & - & 54.7 & 31.3 \\
\hline 6120.249 & $\mathrm{FeI}$ & 44.1 & 63.7 & 41.2 & 46.8 & 46.5 & 36.9 & 48.8 & 59.9 \\
\hline 6137.002 & $\mathrm{FeI}$ & 125.9 & 132.4 & 115.9 & 120.0 & 125.8 & 123.8 & 122.2 & 125.7 \\
\hline 6151.616 & $\mathrm{FeI}$ & 106.7 & 111.4 & 96.4 & 100.3 & 106.2 & 94.8 & 100.8 & 107.2 \\
\hline 6159.382 & $\mathrm{FeI}$ & 40.0 & - & 35.7 & 33.7 & 41.0 & 36.4 & 38.3 & - \\
\hline 6173.340 & FeI & 130.9 & 149.1 & 116.4 & 122.3 & 132.2 & 127.8 & 126.2 & 126.0 \\
\hline 6187.987 & FeI & 87.0 & 99.0 & 80.5 & - & 89.8 & 84.8 & 82.6 & 68.8 \\
\hline 6199.508 & $\mathrm{FeI}$ & 29.4 & - & 23.5 & 26.6 & 32.5 & 21.9 & 34.9 & 25.0 \\
\hline 6200.321 & $\mathrm{FeI}$ & 131.4 & 134.3 & 116.8 & 123.4 & 130.9 & 128.6 & 128.3 & 125.0 \\
\hline 6213.428 & FeI & 144.4 & - & 134.4 & 139.7 & - & 67.2 & 148.6 & 144.5 \\
\hline 6226.730 & $\mathrm{FeI}$ & 72.3 & 63.2 & 67.6 & 64.3 & 73.6 & 110.5 & 61.8 & 56.6 \\
\hline 6240.645 & $\mathrm{FeI}$ & 108.5 & 110.5 & 104.2 & 104.9 & 116.3 & - & 113.1 & 107.8 \\
\hline 6271.283 & FeI & 73.4 & 69.4 & 63.0 & 64.7 & 67.7 & 56.2 & 65.8 & 64.3 \\
\hline 6297.792 & FeI & 136.6 & - & - & - & - & - & 139.2 & 143.7 \\
\hline 6315.813 & $\mathrm{FeI}$ & 96.4 & 86.2 & - & - & - & 75.3 & - & 74.7 \\
\hline 6322.691 & $\mathrm{FeI}$ & 136.8 & 145.6 & 125. & 127.4 & 135.2 & 130.5 & 129.6 & 133.6 \\
\hline 6380.750 & FeI & 100.0 & - & 86.3 & - & - & 97.9 & 102.4 & 74.1 \\
\hline 6385.726 & $\mathrm{FeI}$ & 33.0 & 33.8 & 31.3 & 31.8 & 39.6 & - & 36.0 & 19.0 \\
\hline 6392.538 & $\mathrm{FeI}$ & 69.8 & 74.6 & 63.2 & 69.4 & 75.6 & - & 70.9 & 69.4 \\
\hline 6498.945 & FeI & 117.5 & - & 109.4 & 120.5 & 126.5 & 111.6 & 130.8 & 137.0 \\
\hline 6608.044 & $\mathrm{FeI}$ & 65.7 & 64.4 & 54.3 & 63.5 & 74.9 & 49.8 & 71.5 & 66.6 \\
\hline 6627.560 & $\mathrm{FeI}$ & 66.0 & 69.1 & 59.1 & 53.1 & 64.9 & 48.1 & 62.5 & 38.3 \\
\hline 6633.427 & FeI & 64.4 & 75.3 & 52.9 & 54.5 & 62.8 & 48.0 & 65.8 & 37.1 \\
\hline 6634.123 & $\mathrm{FeI}$ & 90.4 & - & 76.0 & 77.4 & 96.5 & 83.9 & 91.7 & - \\
\hline 6646.966 & $\mathrm{FeI}$ & 50.3 & 71.4 & 43.7 & - & 57.6 & 42.5 & 61.0 & 47.7 \\
\hline 6653.911 & $\mathrm{FeI}$ & 37.9 & 35.4 & 30.0 & - & 39.3 & 29.8 & 40.2 & 24.6 \\
\hline 6696.322 & FeI & 45.4 & 33.9 & 39.6 & 35.5 & 40.8 & 30.0 & 38.2 & 25.2 \\
\hline 6699.136 & $\mathrm{FeI}$ & 30.2 & 38.1 & 29.9 & 26.1 & 31.2 & 24.0 & 34.0 & 17.4 \\
\hline 6703.576 & $\mathrm{FeI}$ & 87.3 & 90.0 & 81.3 & 82.0 & 89.6 & 78.8 & 87.8 & 84.0 \\
\hline 6704.500 & FeI & 24.2 & 31.0 & - & 20.4 & 22.6 & - & 27.7 & 17.3 \\
\hline 6705.105 & $\mathrm{FeI}$ & 86.8 & 93.0 & 79.4 & 76.2 & 86.1 & 76.6 & 84.7 & 56.9 \\
\hline 6710.323 & $\mathrm{FeI}$ & 75.7 & - & 71.5 & 71.8 & 79.7 & 49.6 & 86.4 & 82.1 \\
\hline 6713.745 & $\mathrm{FeI}$ & 51.0 & 50.7 & 46.9 & 41.5 & 48.3 & 42.3 & 46.2 & 30.3 \\
\hline 6725.364 & FeI & 51.5 & 47.2 & 43.6 & 41.0 & 46.1 & 36.3 & 45.4 & 35.4 \\
\hline 6726.673 & $\mathrm{FeI}$ & 81.2 & 73.1 & 73.8 & 69.5 & 77.8 & 68.7 & 74.4 & 57.5 \\
\hline 6732.068 & $\mathrm{FeI}$ & 25.1 & 30.6 & - & - & - & 23.3 & - & - \\
\hline 6733.153 & FeI & 61.0 & 66.6 & 54.3 & 49.7 & 60.2 & 50.2 & 56.5 & 35.3 \\
\hline 6739.524 & FeI & 58.6 & 69.7 & 53.9 & 56.9 & 60.6 & 35.3 & 61.0 & 68.0 \\
\hline 6745.113 & $\mathrm{FeI}$ & 31.0 & 35.1 & 28.2 & 24.8 & 34.3 & 24.1 & 32.7 & 17.9 \\
\hline 6746.975 & FeI & 26.9 & 29.0 & 25.1 & 27.3 & 32.0 & 21.5 & 31.8 & 24.6 \\
\hline 6750.164 & FeI & 136.0 & 146.6 & 123.1 & 128.5 & 145.7 & 138.7 & 138.3 & 128.0 \\
\hline 6752.716 & $\mathrm{FeI}$ & 78.8 & 89.6 & 69.1 & 70.9 & 84.7 & 67.2 & 80.4 & - \\
\hline 6786.860 & $\mathrm{FeI}$ & 66.9 & 58.3 & 62.4 & 55.4 & 67.5 & 69.5 & 62.3 & 45.7 \\
\hline
\end{tabular}


R. Smiljanic et al.: Abundance analysis of barium and mild barium stars, Online Material p 11

Table 9. continued.

\begin{tabular}{|c|c|c|c|c|c|c|c|c|c|}
\hline$\lambda(\AA)$ & Elem. & $\epsilon$ Vir & HR 5058 & HR 5802 & HR 7321 & HR 8115 & HR 8204 & HD 205011 & HR 8878 \\
\hline 6806.856 & FeI & 93.8 & 111.1 & 90.5 & 87.2 & 104.0 & 83.4 & 97.7 & 83.2 \\
\hline 6810.267 & FeI & 92.0 & 79.4 & 86.5 & 79.4 & 95.2 & 89.7 & 85.7 & 64.6 \\
\hline 6820.374 & FeI & 78.3 & - & - & 69.7 & 81.9 & 70.3 & 76.3 & - \\
\hline 6828.596 & FeI & 100.6 & 97.8 & 92.9 & - & 91.3 & 86.7 & 84.4 & 68.5 \\
\hline 6837.013 & FeI & 49.2 & 37.8 & 39.0 & 36.7 & 44.8 & 31.7 & 40.0 & 28.9 \\
\hline 6839.835 & FeI & 90.1 & 109.9 & 76.2 & 80.4 & 91.8 & 72.2 & 87.0 & 83.3 \\
\hline 6842.689 & $\mathrm{FeI}$ & 78.7 & 72.1 & 68.5 & 64.2 & 72.4 & 70.0 & 65.8 & 52.4 \\
\hline 6843.655 & $\mathrm{FeI}$ & 103.1 & 101.5 & 92.4 & 86.3 & 99.3 & 90.4 & 92.9 & 72.3 \\
\hline 6855.166 & FeI & 110.3 & 105.7 & 99.9 & 98.3 & 112.9 & 108.6 & 105.2 & 84.6 \\
\hline 6855.723 & FeI & 57.7 & - & 49.1 & 50.8 & 60.5 & - & 58.2 & 37.3 \\
\hline 6857.251 & FeI & 58.3 & 45.6 & 48.1 & 48.8 & 53.2 & 42.6 & 52.3 & 39.4 \\
\hline 6858.155 & $\mathrm{FeI}$ & 89.0 & 90.7 & 83.2 & 81.3 & 90.5 & - & 89.9 & 62.0 \\
\hline 6861.945 & $\mathrm{FeI}$ & 73.8 & 91.3 & 63.1 & 68.9 & 79.3 & 58.9 & 78.0 & 63.5 \\
\hline 6862.496 & FeI & 67.5 & 61.6 & 55.4 & 57.2 & 68.6 & 54.9 & 62.9 & 40.6 \\
\hline 4656.981 & FeII & 74.4 & 69.3 & 70.7 & 66.7 & 75.6 & 118.1 & 68.7 & 43.7 \\
\hline 4993.358 & FeII & 71.6 & 65.3 & 58.9 & 63.6 & 79.0 & 115.4 & 69.7 & 52.8 \\
\hline 5197.576 & FeII & 122.4 & - & 106.4 & 109.1 & 121.9 & - & 112.9 & - \\
\hline 5234.630 & FeII & 132.7 & - & 117.0 & 111.1 & 127.3 & 135.1 & 110.6 & 88.7 \\
\hline 5264.812 & FeII & 82.8 & 59.5 & 70.1 & 70.0 & 79.8 & - & 67.8 & 50.1 \\
\hline 5325.560 & FeII & 79.7 & - & 67.4 & 67.3 & 80.3 & 130.1 & 69.8 & 43.2 \\
\hline 5414.075 & FeII & 60.1 & 57.6 & 46.2 & 49.6 & 59.6 & 92.2 & 51.6 & 27.6 \\
\hline 5425.257 & FeII & 74.1 & 69.8 & 60.7 & 61.3 & 71.8 & 118.7 & 62.8 & 42.6 \\
\hline 6149.249 & FeII & 67.2 & 64.7 & 55.3 & 53.9 & 71.0 & 113.5 & 57.4 & - \\
\hline 6247.562 & FeII & 89.5 & 59.0 & 79.2 & 67.2 & 76.5 & 140.1 & 67.5 & 51.3 \\
\hline 6369.463 & FeII & 53.3 & 32.9 & 36.3 & 40.5 & 54.5 & 82.8 & 38.7 & 25.1 \\
\hline 6416.928 & FeII & 75.5 & 56.4 & 67.1 & 63.0 & 78.9 & 116.5 & 65.7 & 43.2 \\
\hline 6456.391 & FeII & 100.0 & 84.3 & 83.9 & 77.7 & 94.79 & - & 82.2 & 55.8 \\
\hline 4792.862 & CoI & 69.9 & 77.6 & 70.7 & 70.4 & 72.1 & 78.7 & 72.4 & 73.5 \\
\hline 4813.479 & CoI & 95.3 & - & 86.2 & 89.2 & 92.7 & 69.9 & 87.5 & 86.9 \\
\hline 5212.691 & CoI & 63.8 & 63.6 & 53.2 & 58.3 & 62.5 & - & 58.4 & 66.0 \\
\hline 5280.629 & CoI & 51.9 & 55.6 & 50.1 & 47.0 & 49.5 & 28.8 & 48.0 & 48.9 \\
\hline 5342.708 & $\mathrm{CoI}$ & 59.7 & 65.0 & 54.3 & 58.2 & 69.6 & 68.4 & 65.5 & 59.2 \\
\hline 5359.192 & $\mathrm{CoI}$ & 26.3 & 27.3 & 26.6 & 23.3 & 26.2 & - & 26.1 & 21.1 \\
\hline 5454.572 & CoI & 42.6 & 47.8 & 34.6 & 40.0 & 45.1 & 43.1 & 42.5 & 34.0 \\
\hline 5647.234 & CoI & 64.6 & 81.9 & 60.1 & 60.8 & 56.4 & 30.7 & 60.8 & 75.7 \\
\hline 6455.001 & CoI & 59.1 & - & 49.8 & - & 50.2 & 32.8 & 46.3 & 53.1 \\
\hline 6632.472 & CoI & 43.5 & 66.3 & 37.1 & 45.4 & 51.5 & 36.2 & 55.4 & 55.9 \\
\hline 4935.831 & NiI & 95.1 & - & - & 87.8 & 92.9 & - & - & 80.1 \\
\hline 4946.029 & NiI & 59.7 & 66.5 & - & 49.3 & 53.8 & - & 53.2 & 45.1 \\
\hline 4953.200 & NiI & 98.0 & - & - & 92.5 & 93.2 & 96.2 & 91.2 & - \\
\hline 5010.934 & NiI & 87.2 & 82.3 & 79.1 & 79.8 & 87.0 & 91.6 & 81.6 & 67.7 \\
\hline 5032.723 & NiI & 59.5 & 72.9 & - & 54.3 & 60.4 & - & 58.7 & - \\
\hline 5094.406 & $\mathrm{NiI}$ & 68.1 & 72.1 & 57.1 & 58.9 & 66.0 & 62.6 & 61.6 & - \\
\hline 5197.157 & $\mathrm{NiI}$ & 65.1 & 59.5 & 54.8 & 53.6 & 59.4 & 42.7 & 56.7 & - \\
\hline 5220.300 & NiI & 64.3 & 59.5 & 53.0 & 56.8 & 63.6 & 52.6 & 56.5 & 48.7 \\
\hline 5392.330 & NiI & 37.0 & 23.6 & 28.8 & 28.1 & 31.8 & 22.0 & 28.4 & 21.9 \\
\hline 5435.866 & NiI & 104.1 & 112.4 & 94.9 & 100.1 & 107.9 & 104.0 & 103.5 & 102.7 \\
\hline 5452.860 & NiI & 52.2 & 41.3 & - & 42.1 & 49.3 & 42.3 & 44.0 & 28.9 \\
\hline 5494.876 & $\mathrm{NiI}$ & 59.9 & 43.2 & 50.3 & 51.0 & 58.0 & 48.5 & 54.1 & 38.6 \\
\hline 5587.853 & NiI & 110.3 & - & 100.7 & 107.8 & 113.8 & - & 112.0 & 109.6 \\
\hline 5625.312 & NiI & 82.2 & 88.5 & 73.9 & 66.2 & - & 68.7 & 67.8 & 58.5 \\
\hline 5628.354 & $\mathrm{NiI}$ & 55.0 & - & 49.7 & 43.5 & - & - & - & 31.4 \\
\hline 5637.128 & $\mathrm{NiI}$ & 72.9 & 68.7 & 63.4 & 61.7 & 63.9 & 50.2 & 61.5 & 54.8 \\
\hline 5748.346 & $\mathrm{NiI}$ & 85.6 & - & - & 85.1 & 88.3 & - & 86.7 & 84.6 \\
\hline 5846.986 & NiI & 80.9 & 94.3 & 69.6 & 73.4 & 76.0 & 58.0 & 77.0 & 85.9 \\
\hline 6086.276 & $\mathrm{NiI}$ & 83.0 & 79.9 & - & 69.4 & 75.5 & 71.6 & 71.3 & 56.3 \\
\hline 6176.807 & $\mathrm{NiI}$ & 104.7 & 104.4 & 93.1 & 90.0 & 101.4 & 97.5 & 93.4 & 74.5 \\
\hline 6177.236 & $\mathrm{NiI}$ & 61.5 & 75.0 & 51.9 & 54.8 & 61.0 & 43.9 & 58.9 & 59.7 \\
\hline 6186.709 & $\mathrm{NiI}$ & 63.9 & 55.9 & 56.4 & - & 61.0 & 45.2 & 53.7 & 42.3 \\
\hline 6191.187 & $\mathrm{NiI}$ & 133.4 & 145.4 & 121.7 & - & 135.2 & 122.4 & 130.3 & 134.5 \\
\hline 6327.604 & NiI & 106.3 & 128.8 & 95.5 & 99.0 & 105.0 & 91.0 & 104.4 & 113.0 \\
\hline 6635.137 & $\mathrm{NiI}$ & 56.7 & 67.4 & 50.4 & 50.5 & - & 47.1 & - & 34.0 \\
\hline 6767.784 & NiI & 141.6 & 146.0 & 131.9 & 136.1 & - & 149.0 & 141.1 & 140.6 \\
\hline 6772.321 & NiI & 94.1 & 85.9 & 86.6 & 84.8 & - & 90.6 & 88.6 & 71.0 \\
\hline 6842.043 & $\mathrm{NiI}$ & 70.7 & 64.2 & 60.0 & 56.3 & 64.0 & 48.6 & 59.7 & 50.4 \\
\hline 5218.209 & $\mathrm{CuI}$ & 89.2 & 90.2 & 80.3 & 83.8 & 92.3 & 93.2 & 86.8 & 81.3 \\
\hline 5220.086 & $\mathrm{CuI}$ & 48.7 & 87.1 & 47.1 & 55.9 & 61.8 & - & 65.7 & 43.4 \\
\hline
\end{tabular}


R. Smiljanic et al.: Abundance analysis of barium and mild barium stars, Online Material p 12

Table 9. continued.

\begin{tabular}{|c|c|c|c|c|c|c|c|c|c|}
\hline$\lambda(\AA)$ & Elem. & $\epsilon \mathrm{Vir}$ & HR 5058 & HR 5802 & HR 7321 & HR 8115 & HR 8204 & HD 205011 & HR 8878 \\
\hline 5782.136 & $\mathrm{CuI}$ & 187.4 & 241.1 & 183.9 & 194.5 & 216.8 & 187.6 & 204.4 & 183.0 \\
\hline 4810.537 & $\mathrm{ZnI}$ & 95.4 & 83.3 & 87.4 & 87.4 & 97.5 & 137.3 & 88.6 & 75.0 \\
\hline 4607.338 & SrI & 94.4 & 191.8 & 119.0 & 116.5 & 121.4 & 214.8 & 137.9 & 105.3 \\
\hline 4883.690 & YII & 112.1 & 226.7 & 130.9 & 130.4 & 143.2 & 334.5 & 182.6 & 110.0 \\
\hline 4900.124 & YII & 104.5 & 192.1 & 126.8 & 106.2 & 132.7 & _- & 178.8 & 98.5 \\
\hline 5087.426 & YII & 100.5 & 147.1 & 112.8 & 109.6 & 125.9 & 310.9 & 136.0 & 88.7 \\
\hline 5200.415 & YII & 88.4 & 134.0 & 102.9 & 101.9 & 111.9 & 282.7 & 122.4 & - \\
\hline 5289.820 & YII & 26.9 & 77.4 & 48.7 & 43.6 & 50.4 & 120.5 & 60.9 & 20.3 \\
\hline 5402.780 & YII & 46.5 & 84.2 & 61.9 & 62.2 & 70.8 & 192.7 & - & 42.1 \\
\hline 4739.454 & $\mathrm{ZrI}$ & 44.3 & 120.1 & 73.7 & 70.2 & 69.5 & 124.0 & 94.3 & 72.3 \\
\hline 4613.921 & ZrII & 83.2 & 108.4 & 96.1 & 91.9 & 100.1 & 145.1 & 103.0 & 69.4 \\
\hline 5112.279 & ZrII & 43.2 & 95.8 & 64.6 & 59.0 & 65.4 & 149.5 & 72.5 & 34.2 \\
\hline 5853.688 & BaII & 128.9 & 270.1 & 136.6 & 174.4 & 176.1 & 359.4 & 212.9 & 117.6 \\
\hline 6141.727 & BaII & 218.6 & 497.8 & 258.4 & 271.8 & 287.3 & 613.6 & 386.5 & 187.8 \\
\hline 6496.908 & BaII & 229.0 & 408.7 & 225.7 & 228.7 & 268.4 & 535.0 & 326.3 & 179.3 \\
\hline 4662.512 & LaII & 54.1 & 118.8 & 68.9 & 75.5 & 77.3 & 193.5 & 92.3 & 57.4 \\
\hline 4748.737 & LaII & 32.3 & 74.3 & 43.7 & 43.3 & 44.9 & 110.5 & 55.9 & 28.8 \\
\hline 6320.429 & LaII & 48.6 & 147.6 & 54.2 & 57.6 & 64.8 & 194.1 & 89.3 & 44.7 \\
\hline 6390.493 & LaII & 32.3 & 126.1 & 48.9 & 54.7 & 69.6 & 140.8 & 83.4 & 34.7 \\
\hline 4523.080 & CeII & 74.4 & 147.1 & 83.2 & 95.4 & 100.9 & 287.8 & 113.2 & 85.6 \\
\hline 4562.367 & CeII & 74.4 & 116.3 & 78.2 & 84.2 & 90.0 & 191.1 & 100.0 & 70.4 \\
\hline 4628.160 & CeII & 72.3 & 171.0 & 90.3 & 93.8 & 98.6 & 229.9 & 117.4 & 69.5 \\
\hline 4773.959 & CeII & 39.7 & 75.3 & 41.8 & 51.9 & 55.0 & 119.1 & 61.8 & 37.0 \\
\hline 5274.236 & CeII & 44.8 & 89.2 & 51.6 & 59.1 & 63.1 & 138.3 & 68.9 & 31.9 \\
\hline 5089.831 & NdII & 29.1 & 83.7 & 30.4 & 38.9 & 45.2 & 102.6 & 53.3 & 45.5 \\
\hline 5319.820 & NdII & 57.3 & 103.0 & 65.2 & 73.3 & 77.3 & 180.9 & 83.1 & 66.3 \\
\hline 4566.233 & SmII & 49.3 & 85.7 & 47.4 & 54.5 & 55.5 & 98.4 & 61.4 & 52.1 \\
\hline 6645.127 & EuII & 29.7 & 57.0 & 27.8 & - & 36.4 & 69.8 & 39.2 & 36.6 \\
\hline 5419.880 & GdII & 14.1 & 12.1 & - & 9.4 & 12.6 & - & 13.5 & 7.4 \\
\hline
\end{tabular}


R. Smiljanic et al.: Abundance analysis of barium and mild barium stars, Online Material p 13

Table 10. $\log g f$ s for each component of the lines with hyperfine structure.

\begin{tabular}{|c|c|c|c|c|c|}
\hline 4739.113 & MnI & 5413.684 & $\mathrm{MnI}$ & 5420.350 & MnI \\
\hline$\lambda(\AA)$ & $\log g f$ & $\lambda(\AA)$ & $\log g f$ & $\lambda(\AA)$ & $\log g f$ \\
\hline 4739.099 & -1.269 & 5413.613 & -1.716 & 5420.277 & -2.121 \\
\hline 4739.113 & -1.410 & 5413.653 & -0.921 & 5420.301 & -2.043 \\
\hline 4739.126 & -1.565 & 5413.679 & -1.169 & 5420.334 & -2.903 \\
\hline 4739.145 & -1.122 & 5413.714 & -0.805 & 5420.376 & -1.796 \\
\hline 4739.167 & -2.472 & 5413.722 & -1.265 & 5420.429 & -1.708 \\
\hline 5432.548 & MnI & 5537.765 & MnI & 6013.497 & MnI \\
\hline$\lambda(\AA)$ & $\log g f$ & $\lambda(\AA)$ & $\log g f$ & $\lambda(\AA)$ & $\log g f$ \\
\hline 5432.512 & -3.928 & 5537.691 & -2.689 & 6013.474 & -0.484 \\
\hline 5432.540 & -4.034 & 5537.710 & -2.574 & 6013.486 & -0.694 \\
\hline 5432.565 & -4.144 & 5537.798 & -2.538 & 6013.501 & -0.835 \\
\hline 5432.584 & -4.289 & 5537.764 & -2.574 & 6013.519 & -0.504 \\
\hline 5432.598 & -4.383 & 5537.802 & -2.210 & 6013.537 & -1.082 \\
\hline 6021.803 & MnI & 4792.862 & CoI & 4813.479 & $\mathrm{CoI}$ \\
\hline$\lambda(\AA)$ & $\log g f$ & $\lambda(\AA)$ & $\log g f$ & $\lambda(\AA)$ & $\log g f$ \\
\hline 6021.764 & -1.166 & 4792.811 & -2.165 & 4813.428 & -1.406 \\
\hline 6021.780 & -1.017 & 4792.827 & -1.512 & 4813.451 & -0.929 \\
\hline 6021.797 & -0.186 & 4792.840 & -1.004 & 4813.469 & -0.385 \\
\hline 6021.806 & -0.403 & 4792 & -0.582 & 4813.481 & -0.319 \\
\hline 6021.814 & -0.268 & 4792.864 & -0.545 & 4813.492 & -0.448 \\
\hline 5212.691 & $\mathrm{CoI}$ & 5280.629 & CoI & 5342.708 & $\mathrm{CoI}$ \\
\hline$\lambda(\AA)$ & $\log g f$ & $\lambda(\AA)$ & $\log g f$ & $\lambda(\AA)$ & $\log g f$ \\
\hline 5212.614 & -1.573 & 5280.581 & -1.507 & 5342.647 & -0.247 \\
\hline 5212.856 & -0.866 & 5280.607 & -0.718 & 5342.690 & -0.322 \\
\hline 5212.685 & -0.735 & 5280.629 & -0.851 & 5342.724 & -0.408 \\
\hline 5212.724 & -0.914 & 5280.650 & -0.694 & 5342.751 & -0.513 \\
\hline 5212.759 & -0.902 & 5280.672 & -0.759 & 5342.776 & -0.460 \\
\hline 5359.192 & $\mathrm{CoI}$ & 5454.572 & $\mathrm{CoI}$ & 5647.234 & CoI \\
\hline$\lambda(\AA)$ & $\log g f$ & $\lambda(\AA)$ & $\log g f$ & $\lambda(\AA)$ & $\log g f$ \\
\hline 5359.115 & -0.542 & 5454.529 & -0.397 & 5647.191 & -2.098 \\
\hline 5359.158 & -0.617 & 5454.572 & -0.472 & 5647.200 & -2.173 \\
\hline 5359.192 & -0.703 & 5454.606 & -0.558 & 5647.234 & -2.259 \\
\hline 5359.219 & -0.808 & 5454.633 & -0.663 & 5647.261 & -2.364 \\
\hline 5359.244 & -0.755 & 5454.658 & -0.610 & 5647.291 & -2.311 \\
\hline 6455.001 & $\mathrm{CoI}$ & 6632.472 & CoI & 5218.209 & $\mathrm{CuI}$ \\
\hline$\overline{\lambda(\check{A})}$ & $\log g f$ & $\overline{\lambda(\AA)}$ & $\log g f$ & $\lambda(\check{A})$ & $\log g f$ \\
\hline 6454.931 & -1.447 & 6632.395 & -2.398 & 5218.059 & -1.356 \\
\hline 6454.979 & -0.658 & 6632.438 & -2.473 & 5218.061 & -0.880 \\
\hline 6455.001 & -0.791 & 6632.472 & -2.559 & 5218.063 & -1.010 \\
\hline 6455.022 & -0.634 & 6632.499 & -2.664 & 5218.065 & -0.290 \\
\hline 6455.044 & -0.699 & 6632.524 & -2.611 & 5218.069 & -0.512 \\
\hline - & - & - & - & 5218.071 & -0.512 \\
\hline- & - & - & - & 5218.074 & -0.166 \\
\hline 5220.086 & $\mathrm{Cu} 1$ & 5782.136 & $\mathrm{Cu} 1$ & - & - \\
\hline$\lambda(\AA)$ & $\log g f$ & $\lambda(\AA)$ & $\log g f$ & - & - \\
\hline 5220.080 & -2.086 & 5782.032 & -3.154 & - & - \\
\hline 5220.082 & -1.610 & 5782.042 & -3.457 & - & - \\
\hline 5220.084 & -1.740 & 5782.054 & -2.756 & - & - \\
\hline 5220.086 & -1.031 & 5782.064 & -2.807 & - & - \\
\hline 5220.090 & -1.242 & 5782.073 & -3.111 & - & - \\
\hline 5220.092 & -1.242 & 5782.084 & -2.410 & - & - \\
\hline 5220.095 & -0.916 & 5782.086 & -2.756 & - & - \\
\hline - & - & 5782.098 & -2.756 & - & - \\
\hline - & - & 5782.113 & -2.410 & - & - \\
\hline - & - & 5782.124 & -2.410 & - & - \\
\hline - & _- & 5782.153 & -2.309 & - & - \\
\hline - & - & 5782.173 & -1.963 & - & - \\
\hline
\end{tabular}

